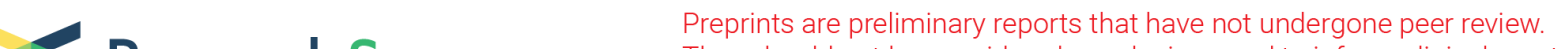 $\begin{array}{ll}\text { Research Square } & \begin{array}{l}\text { They should not be considered conclusive, used to inform clinical practice, } \\ \text { or referenced by the media as validated information. }\end{array}\end{array}$
}

\section{Association of B cell profile and receptor repertoire with the progression of Alzheimer's disease}

\author{
Inhee Mook-Jung ( $\square$ inhee@snu.ac.kr) \\ College of Medicine, Seoul National University https://orcid.org/0000-0001-7085-4085

\section{Jong-Chan Park}

Department of Biochemistry and Biomedical Sciences, Seoul National University, College of Medicine, Seoul https://orcid.org/0000-0001-7516-7292

\section{Jinsung Noh}

Seoul National University https://orcid.org/0000-0002-7167-8113

\section{Sukjin Jang}

Department of Medicine, Seoul National University College of Medicine, Seoul, 03080,

\section{Ki Hyun Kim}

Seoul National University, College of Medicine

\section{Hayoung Choi}

Seoul National University, College of Medicine

\section{Dongjoon Lee}

Department of Biochemistry and Biomedical Sciences, Seoul National University, College of Medicine, Seoul

\section{Junho Chung}

Seoul National University College of Medicine

\section{Dong Young Lee}

6Institute of Human Behavioral Medicine, Medical Research Center, Seoul National University, Seoul, 03080

\section{Yonghee Lee}

Department of Electrical and Computer Engineering, Seoul National University https://orcid.org/00000001-8095-408X

\section{Hyunho Lee}

Department of Electrical Engineering and Computer Science, Seoul National University

\section{Duck Kyun Yoo}

Department of Biochemistry and Molecular Biology, Seoul National University College of Medicine, Seoul, 00380

\section{Amos Lee}

Seoul National University

\section{Min Soo Byun}




\section{Dahyun Yi}

Institute of Human Behavioral Medicine, Medical Research Center Seoul National University, Seoul 03080

\section{Sun-Ho Han}

Department of Biochemistry and Biomedical Sciences, Seoul National University, College of Medicine, Seoul

\section{Sunghoon Kwon}

Seoul National University https://orcid.org/0000-0003-3514-1738

\section{Article}

Keywords: Alzheimer's disease, B lymphocyte, B cell receptor repertoire, Pittsburgh compound B, cerebral amyloid deposition

Posted Date: March 31st, 2021

DOl: https://doi.org/10.21203/rs.3.rs-312269/v1

License: (c) (i) This work is licensed under a Creative Commons Attribution 4.0 International License. Read Full License 


\section{Association of B cell profile and receptor repertoire with the progression of Alzheimer's disease}

Jong-Chan Park, Ph.D. ${ }^{1,2,3,4, \dagger}$, Jinsung Noh ${ }^{5, \dagger}$, Sukjin Jang ${ }^{6, \dagger}$, Ki Hyun Kim, Ph.D. ${ }^{7,8}$, Hayoung Choi ${ }^{1,3}$, Dongjoon Lee ${ }^{1,3}$, Junho Chung, MD, PhD. ${ }^{7,8,9}$, Dong Young Lee, MD, Ph.D. ${ }^{10,11,12}$, Yonghee Lee ${ }^{5}$, Hyunho Lee ${ }^{5}$, Duck Kyun Yoo ${ }^{7,9}$, Amos Chungwon Lee, Ph.D. ${ }^{13}$, Min Soo Byun, MD, Ph.D. ${ }^{14}$, Dahyun Yi, Ph.D. ${ }^{12}$, Sun-Ho Han, Ph.D. 1,2,3,*, Sunghoon Kwon, Ph.D. 5,13,15,16,17,18,*, and Inhee Mook-Jung, Ph.D. 1,2,3,*

†These authors contributed equally to this work.

\section{Abstract}

Alzheimer's disease (AD) is the most common type of dementia. Numerous reports have revealed that peripheral immune systems are linked to neuropathology; however, little is known about the contribution of B lymphocytes in AD. For this longitudinal study, one hundred-thirty-three participants were included in both baseline and follow-up $2^{\text {nd }}$ year. Next, we performed next-generation sequencing (NGS)-based B cell receptor (BCR) repertoire profiling followed by pair-wise overlap analysis. The longitudinal change in the B lymphocytes population was associated with increased cerebral amyloid deposition. Furthermore, patients with AD shared highly similar class-switched BCR sequences with identical isotypes despite the high somatic hypermutation rate of BCR sequences. These commonalities of BCR repertoires of the patients with $\mathrm{AD}$ show the possibility of immunological stimuli by amyloid precursor protein (APP). Thus, we provide evidence for both quantitative and qualitative changes in $\mathrm{B}$ lymphocytes during $\mathrm{AD}$ pathogenesis. The genetic information of patients from the NGS-based BCR repertoire profiling can lead to the development of immune-based therapeutics and treatments for AD. 


\section{Author affiliations:}

${ }^{1}$ Department of Biochemistry and Biomedical Sciences, College of Medicine, Seoul National University, Seoul, 03080, Republic of Korea

${ }^{2}$ Neuroscience Research Institute, Medical Research Center, College of Medicine, Seoul National University, Seoul, 03080, Republic of Korea

${ }^{3}$ SNU Dementia Research Center, College of Medicine, Seoul National University, Seoul, 03080, Republic of Korea

${ }^{4}$ Department of Neurodegenerative Disease, UCL Queen Square Institute of Neurology, University College London, London, WC1N 3BG, United Kingdom

${ }^{5}$ Department of Electrical and Computer Engineering, Seoul National University, Seoul, 08826, Republic of Korea

${ }^{6}$ Department of Medicine, Seoul National University College of Medicine, Seoul, 03080, Republic of Korea

${ }^{7}$ Department of Biochemistry and Molecular Biology, Seoul National University College of Medicine, Seoul, 03080, Republic of Korea

${ }^{8}$ Cancer Research Institute, Seoul National University College of Medicine, Seoul, 03080, Republic of Korea

${ }^{9}$ Department of Biomedical Science, Seoul National University College of Medicine, Seoul, 03080, Republic of Korea

${ }^{10}$ Institute of Human Behavioral Medicine, Medical Research Center, Seoul National University, Seoul, 03080, Republic of Korea

${ }^{11}$ Department of Psychiatry, College of medicine, Seoul National University, Seoul, 03080, Republic of Korea

${ }^{12}$ Department of Neuropsychiatry, Seoul National University Hospital, Seoul, 03080, Republic of Korea

${ }^{13}$ Bio-MAX Institute, Seoul National University, Seoul, 08826, Republic of Korea

${ }^{14}$ Department of Neuropsychiatry, Seoul National University Bundang Hospital, Seongnam, 13620, Republic of Korea 
${ }^{15}$ BK21+ Creative Research Engineer Development for IT, Seoul National University, Seoul, 08826, Republic of Korea

${ }^{16}$ Biomedical Research Institute, Seoul National University Hospital, Seoul, 03080, Republic of Korea

${ }^{17}$ Institutes of Entrepreneurial BioConvergence, Seoul National University, Seoul, 08826, Republic of Korea

${ }^{18}$ Interdisciplinary Program in Bioengineering, Seoul National University, Seoul, 08826, Republic of Korea

\section{Corresponding authors:}

1) Inhee Mook-Jung, Ph.D.

Department of Biochemistry \& Biomedical Sciences, SNU Dementia Research Center Seoul National University College of Medicine

103 Daehak-ro, Jongno-gu, Seoul 03080, South Korea

Tel: +82-2-740-8245; Fax: +82-2-3672-7352; E-mail: inhee@snu.ac.kr

2) Sunghoon Kwon, Ph.D.

Department of Electrical and Computer Engineering

Seoul National University

1 Gwanak-ro, Gwanak-gu, Seoul 08826, South Korea

Tel: +82-2-880-1736; Fax: +82-2-880-1737; E-mail: $\underline{\text { skwon@snu.ac.kr }}$

3) Sun-Ho Han, Ph.D.

Department of Biochemistry \& Biomedical Sciences, Neuroscience Research Institute

Seoul National University College of Medicine

103 Daehak-ro, Jongno-gu, Seoul 03080, South Korea

Tel: +82-2-3668-7640; Fax: +82-2-3672-7352; E-mail: sunho@snu.ac.kr 
Running title: $\mathrm{B}$ cell and its receptor repertoire in $\mathrm{AD}$

Keywords: Alzheimer's disease; B lymphocyte; B cell receptor repertoire; Pittsburgh compound B; cerebral amyloid deposition

Abbreviations: $\mathrm{AD}=$ Alzheimer's disease; $\mathrm{APP}=$ amyloid precursor protein $; \mathrm{BCR}=\mathrm{B}$ cell receptor; NGS = next-generation sequencing; $\mathrm{PBMC}=$ peripheral blood mononuclear cells; $\mathrm{PiB}=$ Pittsburgh compound $\mathrm{B} ; \mathrm{SUVR}=$ standardized uptake value ratio 


\section{Introduction}

Recent researches have shown that Alzheimer's disease (AD) phenotypes are no longer limited to the changes in the brain. There are increasing shreds of evidence that the peripheral immune systems are closely linked to AD pathology, especially critical roles of immune cells in the pathogeneses of $\mathrm{AD}{ }^{1,2}$. So far, however, there has been little discussion about the contribution of blood lymphocytes in AD. Only several papers have revealed the possible association of blood lymphocytes with the decline in cognitive function or accumulation of cerebral amyloid deposition in $\mathrm{AD}$, but these studies have been conducted as a cross-sectional study using lymphocyte population data or failed to correlate specific lymphocyte characteristics with the pathology of $\mathrm{AD}^{3,4,5}$. Besides, although the latest technologies such as $\mathrm{B}$ cell receptor (BCR) or T cell receptor (TCR) next-generation sequencing (NGS) for their repertoire profiling have been developed ${ }^{6,7,8}$, few cases have been applied to the study for $\mathrm{AD} 9$. Especially, no single study exists related to $\mathrm{BCR}$ repertoire analysis in $\mathrm{AD}$. Therefore, this study aims to identify which type of lymphocytes is directly linked with brain amyloid deposition and discover characteristics of BCR repertoires shared among the patients with $\mathrm{AD}$. We found that the population change in B lymphocyte over 2 years was significantly associated with the increase in cerebral $A \beta$ deposition. Furthermore, NGS for B cell receptor followed by pair-wise overlap analysis revealed that the commonalities of BCR repertoires within $\mathrm{AD}$ patients but not within normal participants. We also suggest the possibility that the overlapped BCR sequences might be related to the immunological reaction to amyloid precursor protein (APP). This novel approach through both the longitudinal analysis and $\mathrm{BCR}$ repertoire profiling to $\mathrm{AD}$ provides a perspective of understanding the link between peripheral immune systems and neuropathology in the brain of $\mathrm{AD}$ patients.

\section{Results}

\section{The longitudinal study revealed $B$ lymphocyte population is associated with brain amyloid deposition in AD}

Fig. 1A shows the overall strategy for flow cytometry analysis (Supplementary Fig. 1) followed by the longitudinal analysis for B lymphocyte population changes. For longitudinal 
analyses, we sorted participants into three groups: $\mathrm{PiB}$ decrease group $\left(\mathrm{PiB}^{\mathrm{Dec}}\right), \mathrm{PiB}$ stable group ( $\left.\mathrm{PiB}^{\text {Stable }}\right), \mathrm{PiB}$ increase group $\left(\mathrm{PiB}^{\mathrm{Inc}}\right)$ based on the changes in PiB-PET SUVR for 2 years (Fig. 1A, Table 1). Stepwise multiple regression analysis showed that the population change in $\mathrm{B}$ lymphocyte over 2 years $(\Delta \mathrm{B})$ was significantly associated with the increase in brain $\mathrm{A} \beta$ (change of $\mathrm{PiB}$ standardized uptake value ratio; $\Delta \mathrm{PiB}$ ), but the changes in other lymphocytes $(\Delta \mathrm{T}, \Delta \mathrm{NK})$ were not (Fig. 1B). That was the decisive reason for us to start focusing on the profiling of $\mathrm{B}$ cells. Interestingly, $\mathrm{PiB}^{\mathrm{Inc}}$ had a higher portion of participants with an increasing number of B lymphocytes (Fig. 1C), regardless of the baseline status of $\mathrm{A} \beta$ accumulation (Fig. S2). Furthermore, partial correlation and ANOVA post-hoc analysis showed that $\Delta \mathrm{B}$ was positively correlated with $\triangle \mathrm{PiB}$ (Fig. 2A, left) and $\Delta \mathrm{B}$ quartile four (Q4) had the highest $\Delta \mathrm{PiB}$ value $(\mathrm{Q} 1<\mathrm{Q} 2<\mathrm{Q} 3<\mathrm{Q} 4$; Fig. 2A, right $)$. Moreover, $\mathrm{PiB}^{\text {Inc }}$ had higher $\triangle \mathrm{B}$ than $\mathrm{PiB}^{\mathrm{Dec}}$ and $\mathrm{PiB}^{\text {Stable }}$ (Fig. 2B). Additional ROC curve analysis showed that $\Delta \mathrm{B}$ significantly increases the discrimination power for the PiB increase as a variable for the logistic regression analysis (Fig. 2C). The combination of $\mathrm{ApoE}$ and $\Delta \mathrm{B}$ variable had higher sensitivity (70.6\%) and specificity (72.7\%) with a 0.754 area under curve (AUC) than ApoE alone (0.710 AUC with 55.9\% sensitivity and 76.8\% specificity) (Fig. 2D). To do further validation, we classified participants depending on the $\triangle \mathrm{B}$ quartiles (BQ; $\mathrm{BQ} 1<\mathrm{BQ} 2<\mathrm{BQ} 3<\mathrm{BQ} 4)$, and compare their $\triangle \mathrm{PiB}$. As a result, BQ4 and BQ2-3 groups had higher $\triangle \mathrm{PiB}$ than BQ1 (Fig. 2E). These results led us to focus on B lymphocyte profiling for the following studies.

\section{Pair-wised overlap analysis followed by NGS-based BCR repertoire analysis}

For deep profiling of B lymphocytes in the BCR sequence level, we randomly selected representative 13 participants ( 3 Normal, 10 AD) with even distribution of apolipoprotein $\mathrm{E}$ allele frequencies ( $2 \varepsilon 3 / \varepsilon 3$ and $1 \varepsilon 3 / \varepsilon 4$ for Normal; $3 \varepsilon 3 / \varepsilon 3,4 \varepsilon 3 / \varepsilon 4,3 \varepsilon 4 / \varepsilon 4$ for AD), ages (73.6 \pm 1.9 for Normal, $68.6 \pm 2.2$ for AD), and the number of total lymphocytes $(30.7 \pm 3.4$ for Normal, $31.6 \pm 2.6$ for AD) that can interfere in the analysis for B lymphocyte ${ }^{4,10,11}$ (Fig. 3A). The cerebral amyloid deposition (standardized uptake value ratio, SUVR), mini-mental state exam (MMSE) cognitive score, plasma t-tau, and beta-amyloid 42/40 ratio were significantly different between normal and AD, but not age (Fig. 3B-3C). Next, we generated $\mathrm{BCR}$ repertoire data of these samples. In the whole BCR repertoire level, we discovered no distinctive traits shared among the AD samples, probably due to the chronic characteristics of 
the diseases compared to non-chronic characteristics of diseases such as auto-immune or infectious diseases (Supplementary Fig. 3 to 6) ${ }^{12}$. Thus, to discover characteristics shared among the AD samples, we focused our analysis on a subset of the whole BCR repertoire overlapped among the samples. Due to the limited number of BCR sequences shared among more than two patients, we conducted the overlap analysis in a pairwise manner. Overlapped BCR sequences were categorized into two groups, Normal\&X (comparison between Normal and Normal or Normal and $\mathrm{AD}$ ) and $\mathrm{AD \& AD}$ (comparison between $\mathrm{AD}$ and $\mathrm{AD}$ ) (Fig. 3A, 3D left), according to overlap pattern. We hypothesized that overlap between Normal and AD has an identical pattern with that between Normal and Normal because the two groups of samples do not share common immunological stimuli. Thus, we defined the two comparisons collectively as Normal\&X and compared it with AD\&AD. Interestingly, AD\&AD had more numbers of the overlapped BCR sequences and lineages compared to Normal\&X (Fig. 3D, right). However, in divergence and isotype composition, the two groups showed no significant difference (Fig. 3E-3F).

\section{Pair-wised overlap analysis with highly similar overlapped BCR sequences revealed the commonalities of its repertoires within AD \\ patients}

To exclude coincidently defined as the overlapped BCR sequences and investigate overlapped BCR sequences in a stricter aspect, we calculated inter-sample full amino acid (AA) distance (shorter distance means higher similarity) of the overlapped BCR sequences ${ }^{13}$. We defined the overlapped BCR sequences by VJ gene usage (related to the mechanism of somatic recombination) ${ }^{14}$ and complementarity-determining region 3 (CDR3; the most variable portion of Ig molecules) ${ }^{15}$ AA sequence homology (> 70\%) as previously reported 13, 16. Also, due to the limited resolution of the used criteria, BCR sequences with distinctive somatic hypermutation (SHM; a process diversifying BCRs through point-mutations into Ig genes) patterns ${ }^{17}$ were included in the overlapped BCR sequences. Thus, we quantified the degree of SHM sharing by defining the inter-sample full AA distance as the Hamming distance calculated in full AA level between the overlapped BCR sequences from the different samples ${ }^{18}$. AD\&AD showed a shorter inter-sample full AA distance compared to Normal\&X (Fig. 4A, left). 
Then, the sub-population of the overlapped BCR sequences with small inter-sample full AA distance $(<5)$ was extracted and defined as highly similar overlapped BCR sequences. These highly similar overlapped BCR sequences can be generated in two ways. First, for naïve B lymphocytes, gene-rearrangement could generate coincidentally similar CDR3 with some bias inherent in the process. These sequences would have a very small number of SHMs and be in IgM isotype ${ }^{6,19}$. Second, for activated B lymphocytes, induction of somatic mutations by antigens can produce converged BCR sequences ${ }^{20,21}$. In this case, the sequences have high divergence values and are class-switched to isotypes other than IgM. These highly similar overlapped $\mathrm{BCR}$ sequences of $\mathrm{AD} \& \mathrm{AD}$ showed distinctive characteristics in the aspect of divergence and isotype composition compared to those of Normal\&X. Among the sequences, sequences with a high divergence despite a small inter-sample full AA distance were only detected in $\mathrm{AD} \& \mathrm{AD}$, which means mutation induction in the same direction resulting in identical residues for binding occurred only in AD\&AD (Fig. 4A, right). Also, the number of Ig counts were significantly increased in AD\&AD group (Fig. 4B). Furthermore, most of these sequences with high divergence values were class-switched to the isotypes other than IgM (Fig. 4C). A total of 579 sequences and 108 lineages was classswitched among the highly similar overlapped BCR sequences. Among 108 lineages, 92 lineages contained more than one class-switched BCR sequence in both repertoires with a distinctive VJ gene utilization compared to whole class-switched repertoires of the samples (Fig. 4D and Supplementary Table 2-3). Strikingly, in 91 out of 92 lineages, BCR sequences were represented with identical isotypes in two different repertoires, which means it is feasible for these sequences to result in the same effector functions. In one lineage with different isotypes, the sequences emerged as IgA1 and IgA2, respectively (Fig. 4E). These identical characteristics of the highly similar and class-switched overlapped sequences were also reproduced in a phylogenetic analysis by confirming that the sequences from different samples were clustered into the same sub-family with identical isotypes (Supplementary Fig. 7). We concluded there is a possible response to the stimulation from the same antigen ${ }^{22,23}$. In other words, the commonalities of BCR repertoires in the patients with AD implied the possibility of immunological stimuli by common antigens.

\section{Estimation of target proteins through in silico mapping analysis}

To estimate the target proteins of the overlapped BCR sequences, we conducted in silico mapping of the overlapped BCR sequences to the BCR sequences, of which 3D structure and 
binding targets were defined. From the IMGT 3D structure database, we could confirm 39 unique BCR sequences and their 3D structure known to bind to AD-related proteins ${ }^{24}$ (Fig. 5). Then, we mapped the $39 \mathrm{BCR}$ sequences binding to the AD-related proteins to the overlapped BCR sequences identified in our analysis while increasing the allowed number of CDR3 amino acid mismatches during the mapping (Fig. 5A). For most of the AD-related protein-binding antibodies, which showed no significant match with the overlapped BCR sequences, there was no difference in mapping patterns between AD\&AD and Normal\&X. However, AD\&AD and Normal\&X showed different mapping patterns for two APP-binding antibodies with the simple CDR3 amino acids sequence of GDY (Fig. 5B-5C, Supplementary Fig. 8-9). A total of 82 overlapped BCR sequences with the perfectly matched CDR3 amino acid sequences were detected in AD\&AD compared to the case of Normal\&X where seven BCR sequences were detected. Also, class-switched overlapped BCR sequences were only detected in AD\&AD (40 out of the 82 overlapped BCR sequences, 48.78\%). This implies the possibility that these overlapped BCR sequences having the CDR3 amino acid sequence of GDY could be related to the immunological reaction to amyloid precursor protein (APP). Fig. 6 shows a graphical summary of this study.

\section{Discussion}

This is the first investigation into the association of $\mathrm{B}$ cell and its receptor repertoire with AD. We provide clear evidence for both quantitative and qualitative changes of B cell during the progression of $\mathrm{AD}$ pathology. Although there have been several reports suggests that the links between the population of $\mathrm{B}$ lymphocyte and pathology of $\mathrm{AD}$ or clinical dementia symptoms, these researches just relied on the cross-sectional study and the results were quite controversial. For example, one prospective cohort study including 105 participants showed no differences in the percentage of B lymphocytes per total PBMC in the patients with AD compared to healthy controls but identified an increased number of B lymphocytes producing autoantibodies against beta-amyloid in $\mathrm{AD}^{25}$. On the other hand, other cross-sectional studies found a decrease in B lymphocytes as well as T lymphocytes in the patients with AD 4, 26, 27 . Even though this discrepancy has been questioned continuously, many studies entertain the concept that lymphocytic abnormality is accompanied by dementia, suggesting that the population of lymphocytes could be a biomarker for $\mathrm{AD}{ }^{28}$. A recent study identified the specific intracellular signaling markers, PLC $\gamma 2$ in stimulated PBMC subsets from AD patients through single cell quantification and machine learning model ${ }^{29}$, however, the 
relevance of specific cell type among PBMC to AD pathology has been still uncertain, especially B cell population. In the present study, our quantitative analyses revealed that the more increase in the number of B lymphocytes, the more brain A $\beta$ deposition occurred. The difference from previous studies is that we investigated the direct relationship with the changes in brain beta-amyloid through our longitudinal analyses. We thought this approach could lead to additional findings that would be difficult to identify in the cross-sectional study, and we revealed the longitudinal association of B lymphocyte with the pathology of AD.

Through our longitudinal study, we realized that it is worth trying to closely monitor the characteristics of B lymphocytes themselves within AD patients. Furthermore, since many of the latest technologies such as unbiased next-generation TCR/BCR repertoire analysis or single-cell sequencing have been developed, we thought it would be interesting to apply these technologies to our cohorts. Thus, we performed NGS-based BCR repertoire analysis for the deep profiling of BCR repertoire, and consequently, our analyses for B lymphocytes in the BCR sequence level identified the commonalities of BCR repertoires within AD patients implying the possibility of immunological stimuli by the common antigens such as plasma beta-amyloid or tau proteins ${ }^{22,23}$. Moreover, we found the possibility that the overlapped BCR sequences matched with the CDR3 amino acid sequence of GDY could be related to the immunological reaction to APP, which is known as the precursor molecule of beta-amyloid.

Our work has some limitations and suggestions for future study. First, the short length of CDR3 and the limited diversity the region could contribute to the prevalence of these BCR sequences. Stringent experimental methods have to be followed to prove the relevance of the mapped BCR sequences with the disease. Second, future works should concentrate to find out the cause of this phenomenon. The other concern is that we performed the BCR repertoire analysis with only 13 participants. A validation study with a larger sample size is needed to confirm these data. Alternatively, further research through the isolated human B lymphocyte culture or induced- pluripotent cell (iPSC) culture could help us find specific antigens stimulating B lymphocytes.

Our approach through both the longitudinal analysis and BCR repertoire profiling to AD suggest that the immunological response of peripheral B lymphocyte is clearly associated with neuropathology in the brain of AD patients. Furthermore, the genetic information of BCR obtained from this study using next-generation repertoire analysis techniques can lead to the development of various immune-based therapeutics and treatments for AD. 


\section{Methods}

\section{Recruitment of participants}

Total 133 participants were included, and they are sorted into three groups for our longitudinal analysis: Pittsburgh compound B-positron emission tomography (PiB-PET; brain PET-imaging for the cerebral amyloid deposition) standardized uptake value ratio (SUVR) decrease group for 2 years ( $\mathrm{PiB}^{\mathrm{Dec}}, \mathrm{n}=33$ ), $\mathrm{PiB}$ stable group ( $\mathrm{PiB}^{\text {Stable }}, \mathrm{n}=66$ ), and $\mathrm{PiB}$ increase group $\left(\mathrm{PiB}^{\mathrm{Inc}}, \mathrm{n}=34\right)$ (Table 1). All of the subjects underwent brain imaging such as structural MRI and PiB-PET as well as psychological or clinical assessments according to the expert's guideline. The assessments corresponded to neuropsychological test based on the Korea Brain Aging Study for the Early diagnosis and prediction of AD (KBASE) protocol including neuropsychological battery (including Mini-Mental State Examination; MMSE), the Consortium to Establish a Registry for Alzheimer`s Disease Assessment Packet (CERAD) and KBASE clinical assessments protocol (CERAD-K) ${ }^{30}$. MMSE z-score was calculated by the normalization of MMSE scores with the appropriate correction for age, gender, and education levels.

\section{Neuroimaging}

PiB-PET imaging and MR imaging were conducted using a simultaneous 3.0 T PET-MR scanner (Biograph mMR scanner; Siemens, Washington DC, USA) as previously reported manner ${ }^{31} .555 \mathrm{MBq}$ of ${ }^{11} \mathrm{C}$-PiB were injected intravenously and PiB-PET emission scan was acquired and the images were analyzed ${ }^{23,32,33,34,35}$. Prominent ${ }^{11} \mathrm{C}$-PiB retention in lateral temporal areas and brain regions has been described ${ }^{36}$ and the regions of interest (ROIs) were reported ${ }^{37}$. A standardized uptake value ratio (SUVR) of each ROI was obtained by normalizing the all voxels mean value of each ROI by the mean values of cerebellar uptake. Participants were divided as $\mathrm{PiB}$-positive $(\mathrm{PiB}+)$ or $\mathrm{PiB}-$ negative $(\mathrm{PiB}-)$ by SUVR value based on the criteria 1.4 in at least one of the four ROIs ${ }^{32,34}$.

\section{Blood sampling, isolation of PBMCs and their storage}

All blood samples were collected in the morning, using K2 EDTA tubes (BD Vacutainer Systems, Plymouth, UK) after overnight fasting. The EDTA tubes were immediately centrifuged and plasma supernatants were removed. The remaining blood was mixed with 
PBS and transferred into Ficoll-Hypauqe solution for isolation of peripheral blood mononuclear cells (PBMC) using gradient through repeating centrifugation and PBS washing. The collected PBMCs were immediately cryopreserved by using cell freezing buffer $(90 \%$ fetal bovine serum $+10 \%$ DMSO) and stored for one week in the Mr.Frosty Freezing Container (Thermo Scientific; Waltham, MA, USA). After a week, the vials moved to the LN2 tank and stored at $-192^{\circ} \mathrm{C}$. The detailed information of used reagents for this study was summarized in Table S1.

\section{Flow cytometry}

Cryopreserved PBMCs have recovered in RPMI 1640 media and then used for flow cytometry (FACS) analysis. After the recovery process, HBSS based FACS buffer containing $2 \%$ FBS and $0.05 \%$ sodium-azide were used. For lymphocyte immunophenotyping, after excluding the pan-myeloid marker (CD33) positive population, remaining lymphocyte populations were identified as pan-T cell marker (CD3), pan-B cell marker (CD19), and panNK marker (CD16) positive populations, respectively. Used fluorescence conjugated antibody information are as indicated in the text: APC anti-human CD33 antibody (1:100, clone WM53), FITC anti-human CD3 antibody (1:100, clone OKT3), PE anti-human CD19 antibody (1:100, clone HIB19), and APC/Cy7 anti-human CD16 antibody (1:100, clone Ts1/18). For immunostaining, cells were passed through a $35 \mu \mathrm{m}$ nylon mesh strainer, blocked for $15 \mathrm{~min}$ on ice with Human TruStain FcX (5 $\mu \mathrm{l} /$ million cells in $100 \mathrm{ul}$ staining volume, BioLegend), and stained for $30 \mathrm{~min}$ on ice with antibody mixture. DAPI staining (0.4 $\mu \mathrm{g} / \mathrm{ml})$ was formed to identify dead cells. Prepared cells were analyzed on a Fortessa X-20 (BD Biosciences) and the data were analyzed using FlowJo software (Tree Star). The detailed information of used reagents for this study was summarized in Supplementary Table 1.

\section{Next-Generation Sequencing (NGS) library preparation}

Cryopreserved $\mathrm{CD} 19^{+} \mathrm{B}$ lymphocytes were thawed and total RNA was extracted using the TRIzol Plus RNA Purification Kit (Life Technologies). $1 \mu \mathrm{g}$ of total RNA was used as input for library preparation. Reverse transcription was performed according to the manufacturer's instructions using SuperScript IV reverse transcriptase (Life Technologies) and primers for five immunoglobulin heavy chain isotypes containing UMI (unique molecular identifiers) barcode consists of 14 random nt and partial Illumina adapters (Supplementary Table 2). Primer annealing was carried out at $72^{\circ} \mathrm{C}$ for 3 min then immediately placed on ice for 2 min. 
The first-strand cDNA was purified using AmPure XP beads (Beckman Coulter) at a 1:1.8 ratio, then second-strand cDNA was synthesized using KAPA HiFi HotStart DNA polymerase (Kappa Bioscience) and a pool of 6 immunoglobulin heavy chain variable regionspecific primers (Supplementary Table 3) $\left(98^{\circ} \mathrm{C}\right.$ for $4 \mathrm{~min}, 52^{\circ} \mathrm{C}$ for $1 \mathrm{~min}, 72^{\circ} \mathrm{C}$ for $5 \mathrm{~min}$ ) 19, 38. Double-stranded cDNA was purified using AmPure XP beads (Beckman Coulter) at a 1:1 ratio, then amplified with KAPA HiFi HotStart DNA polymerase (Kappa Bioscience) using two primers containing Illumina adapters and index sequences $\left(95^{\circ} \mathrm{C}\right.$ for $3 \mathrm{~min}, 25$ cycles of $95^{\circ} \mathrm{C}$ for $30 \mathrm{~s}, 65^{\circ} \mathrm{C}$ for $30 \mathrm{~s}, 72^{\circ} \mathrm{C}$ for $1 \mathrm{~min}$, and $72^{\circ} \mathrm{C}$ for $5 \mathrm{~min}$ ). Final NGS libraries were purified using AmPure XP beads (Beckman Coulter) at a 1:1 ratio, then submitted for a quality control procedure on TapeStation 2200 (Agilent Technologies). Libraries with a single peak of correct sequence length were subjected to NGS analysis using the MiSeq platforms (Illumina, Inc.).

\section{Consensus sequence extraction from raw NGS reads}

The raw NGS reads were q-filtered by the q20p95 condition, which means $95 \%$ nucleotides of the reads have to have higher Phred scores than 20 . On average, $83.51 \%$ of the reads were passed the q-filtering condition. Then, the primer regions used in the experiments were extracted from the reads while allowing one mismatch (substitution, insertion, or deletion). Based on the location of the primer sequences, UMI sequences were extracted and the reads were clustered according to the UMI sequences. The reads in the same UMI clusters were aligned using a multiple sequence alignment tool, the Clustal Omega 1.2.4 ${ }^{39}$, 40 . By using the nucleotides frequency information of the alignment results, the consensus sequence of the UMI clusters was extracted, then the read-count of the sequences was re-defined as the number of unique UMI sequences.

\section{Isotype annotation and functionality filtering of the consensus sequences}

The constant region of the consensus sequences was used for isotype annotation. The constant region sequences were recognized in a location-based manner, then the sequences were aligned to an in-house constant gene database constructed based on the IMGT (the international immunogenetics information system) database ${ }^{41}$. The isotype of the consensus sequences was determined by using the alignment results. Then, the V/D/J genes and 
CDR1/2/3 regions of the sequences were extracted and annotated by the IgBLAST 1.8.0 ${ }^{42}$. After the annotation, non-functional consensus sequences were filtered-out by the following condition. 1. Shorter sequence length than $250 \mathrm{bp}, 2$. Existence of stop-codon or frame-shift in full AA sequences, 3. Annotation failure in more or equal to one of the CDR1/2/3 regions.

\section{Divergence from germline $\mathrm{V}$ gene segments calculation}

As the first step of the divergence calculation, the BCR sequences were aligned to germline variable gene segments by using IgBLAST ${ }^{42}$. Based on the IgBLAST alignment results, the aligned length of the sequences to the gene segments and the number of mutations within the aligned region were calculated. We defined the divergence value as the number of mutations divided by the aligned length as reported previously ${ }^{6}$

\section{Pair-wise overlap analysis and inter-sample full AA distance calculation}

Pair-wise overlap analysis was conducted at the BCR lineage level. To define BCR lineages, a previously reported hierarchical clustering method was used ${ }^{18}$. BCR lineages satisfying the following conditions from two different repertoire data were merged and defined as overlapped BCR lineages. 1. Same V/J gene usage, 2. Same CDR3 sequence length, 3. CDR3 AA sequence homology more than 0.7. In the above condition, CDR3 AA sequence homology was set as one minus CDR3 AA sequence discrepancy, which was calculated by minimum Hamming distance in CDR3 AA sequence between all the combinations of BCR sequences from two different BCR lineages divided by CDR3 AA sequence length. BCR sequences of the overlapped BCR lineages were defined as overlapped BCR sequences. To define inter-sample full AA distance, BCR sequences in the same overlapped BCR lineages were used. First, the full AA sequence of BCR sequences in the same overlapped BCR lineages was trimmed and aligned. Then, Hamming distance in the full AA sequence between all the combinations of BCR sequences from two different samples was calculated. Among the calculated Hamming distance values, the minimum value was set as an inter-sample full AA distance value of the overlapped BCR lineage. 


\section{In silico mapping of the overlapped BCR sequences to antibodies binding to AD-related proteins}

First, we defined $\mathrm{V}_{\mathrm{H}}$ sequences of the antibodies binding to AD-related proteins using the IMGT 3D structure database, which led to 39 unique $\mathrm{V}_{\mathrm{H}}$ sequences ${ }^{43}$. Then, we mapped the identified $\mathrm{V}_{\mathrm{H}}$ sequences to the overlapped BCR sequences of our analysis based on the identity of CDR3 amino acid sequences. The overlapped BCR sequences having the same CDR3 length as that of the $\mathrm{V}_{\mathrm{H}}$ sequence of antibodies binding to AD-related proteins were identified, and CDR3 mismatch was defined as the Hamming distance between their CDR3 amino acid sequences. The CDR3 amino acid mismatch threshold was defined as the number of allowed mismatches divided by the length of CDR3 amino acid sequences. The number of mapped $V_{H}$ sequences was normalized by the number of $V_{H}$ sequences with the same CDR3 length and the normalized values were defined as the mapping efficiency. This means that when the CDR3 amino acid mismatch threshold has a value of 1.0, mapping efficiency can only have a value of 0.0 or 1.0 .

\section{Statistical analyses}

All statistical analyses were performed by GraphPad Prism 8 (San Diego, CA, USA) or Medcalc Software (Acacialaan, Ostend, Belgium). Categorical data were analyzed using the chi-square test. Independent $t$-test and ANOVA post-hoc test were used for the comparison of numerical data. The outliers were excluded by the Grubb's test. The association between the values was determined by partial correlation analyses, with the correction for the several covariates. Multiple regression analysis was also performed. To test the discrimination power of variables, logistic regression followed by receiver operating characteristic (ROC) curve analysis was conducted. For comparison of numerical values used in the pair-wise overlap analysis, the Wilcoxon rank-sum test was conducted after the Shapiro Wilk test.

\section{Ethical approval}

This study was approved under the recommendations of the Institutional Review Board (IRB) of the Seoul National University Hospital (SNUH), South Korea. Written informed consent was applied to all the participants, corresponding to the Declaration of Helsinki. Protocols and manuals were also approved by the IRB of the SNUH. 


\section{Reporting Summary}

Available soon.

\section{Data availability}

The sequencing data from this study are available at NCBI under SRA accession number PRJNA667860 (https://www.ncbi.nlm.nih.gov/bioproject/?term=PRJNA667860). Also, all other data are available upon reasonable request.

\section{Code availability}

Non-applicable.

\section{References}

1. Goldeck D, Witkowski JM, Fulop T, Pawelec G. Peripheral Immune Signatures in Alzheimer Disease. Curr Alzheimer Res 13, $739-749$ (2016).

2. Park JC, Han SH, Mook-Jung I. Peripheral inflammatory biomarkers in Alzheimer's disease: a brief review. $B M B$ Rep 53, 10-19 (2020).

3. Poinsatte $\mathrm{K}$, et al. $\mathrm{T}$ and $\mathrm{B}$ cell subsets differentially correlate with amyloid deposition and neurocognitive function in patients with amnestic mild cognitive impairment after one year of physical activity. Exerc Immunol Rev 25, 34-49 (2019).

4. Richartz-Salzburger E, et al. Altered lymphocyte distribution in Alzheimer's disease. J Psychiatr Res 41, 174-178 (2007).

5. Cao W, Zheng H. Peripheral immune system in aging and Alzheimer's disease. Mol Neurodegener 13, 51 (2018).

6. Soto C, et al. High frequency of shared clonotypes in human B cell receptor repertoires. Nature 566, 398-402 (2019).

7. Bashford-Rogers RJM, et al. Analysis of the B cell receptor repertoire in six immunemediated diseases. Nature 574, 122-126 (2019).

8. Davis CW, et al. Longitudinal Analysis of the Human B Cell Response to Ebola Virus Infection. Cell 177, 1566-1582 e1517 (2019). 
9. Gate D, et al. Clonally expanded CD8 $\mathrm{T}$ cells patrol the cerebrospinal fluid in Alzheimer's disease. Nature 577, 399-404 (2020).

10. Allan LL, et al. Apolipoprotein-mediated lipid antigen presentation in B cells provides a pathway for innate help by NKT cells. Blood 114, 2411-2416 (2009).

11. Frasca D, Blomberg BB. Effects of aging on B cell function. Curr Opin Immunol 21, 425-430 (2009).

12. Kim SI, et al. Stereotypic neutralizing VH antibodies against SARS-CoV-2 spike protein receptor binding domain in patients with COVID-19 and healthy individuals. Sci Transl Med 13, (2021).

13. Setliff I, et al. Multi-Donor Longitudinal Antibody Repertoire Sequencing Reveals the Existence of Public Antibody Clonotypes in HIV-1 Infection. Cell Host Microbe 23, 845-854 e846 (2018).

14. Roth DB. V(D)J Recombination: Mechanism, Errors, and Fidelity. Microbiol Spectr 2, (2014).

15. VanDyk L, Meek K. Assembly of IgH CDR3: mechanism, regulation, and influence on antibody diversity. Int Rev Immunol 8, 123-133 (1992).

16. Ehrhardt SA, et al. Polyclonal and convergent antibody response to Ebola virus vaccine rVSV-ZEBOV. Nat Med 25, 1589-1600 (2019).

17. Cui A, et al. A Model of Somatic Hypermutation Targeting in Mice Based on HighThroughput Ig Sequencing Data. J Immunol 197, 3566-3574 (2016).

18. Gupta NT, Adams KD, Briggs AW, Timberlake SC, Vigneault F, Kleinstein SH. Hierarchical Clustering Can Identify B Cell Clones with High Confidence in Ig Repertoire Sequencing Data. J Immunol 198, 2489-2499 (2017).

19. Briney B, Inderbitzin A, Joyce C, Burton DR. Commonality despite exceptional diversity in the baseline human antibody repertoire. Nature 566, 393-397 (2019).

20. Jackson KJ, et al. Human responses to influenza vaccination show seroconversion signatures and convergent antibody rearrangements. Cell Host Microbe 16, 105-114 (2014).

21. Parameswaran $\mathrm{P}$, et al. Convergent antibody signatures in human dengue. Cell Host Microbe 13, 691-700 (2013). 
22. Park JC, et al. Plasma tau/amyloid-beta1-42 ratio predicts brain tau deposition and neurodegeneration in Alzheimer's disease. Brain 142, 771-786 (2019).

23. Park JC, et al. Chemically treated plasma Abeta is a potential blood-based biomarker for screening cerebral amyloid deposition. Alzheimers Res Ther 9, 20 (2017).

24. Seo J, et al. Genetic associations of in vivo pathology influence Alzheimer's disease susceptibility. Alzheimers Res Ther 12, 156 (2020).

25. Sollvander S, et al. Increased Number of Plasma B Cells Producing Autoantibodies Against Abeta42 Protofibrils in Alzheimer's Disease. J Alzheimers Dis 48, 63-72 (2015).

26. Tavolato B, Argentiero V. Immunological indices in presenile Alzheimer's disease. $J$ Neurol Sci 46, 325-331 (1980).

27. Tollefson GD, Godes M, Warren JB, Haus E, Luxenberg M, Garvey M. Lymphopenia in primary degenerative dementia. J Psychiatr Res 23, 191-199 (1989).

28. Rezai-Zadeh K, Gate D, Szekely CA, Town T. Can peripheral leukocytes be used as Alzheimer's disease biomarkers? Expert Rev Neurother 9, 1623-1633 (2009).

29. Phongpreecha $\mathrm{T}$, et al. Single-cell peripheral immunoprofiling of Alzheimer's and Parkinson's diseases. Sci Adv 6, (2020).

30. Byun MS, et al. Korean Brain Aging Study for the Early Diagnosis and Prediction of Alzheimer's Disease: Methodology and Baseline Sample Characteristics. Psychiatry Investig 14, 851-863 (2017).

31. Han $\mathrm{SH}$, et al. Blood acetylcholinesterase level is a potential biomarker for the early detection of cerebral amyloid deposition in cognitively normal individuals. Neurobiol Aging 73, 21-29 (2019).

32. Reiman EM, et al. Fibrillar amyloid-beta burden in cognitively normal people at 3 levels of genetic risk for Alzheimer's disease. Proc Natl Acad Sci U S A 106, 68206825 (2009).

33. Jack CR, Jr., et al. 11C PiB and structural MRI provide complementary information in imaging of Alzheimer's disease and amnestic mild cognitive impairment. Brain 131, 665-680 (2008). 
34. Choe YM, et al. Association of homocysteine with hippocampal volume independent of cerebral amyloid and vascular burden. Neurobiol Aging 35, 1519-1525 (2014).

35. Weiss SJ. Tissue destruction by neutrophils. N Engl J Med 320, 365-376 (1989).

36. Klunk WE, et al. Imaging brain amyloid in Alzheimer's disease with Pittsburgh Compound-B. Ann Neurol 55, 306-319 (2004).

37. Yaffe $\mathrm{K}$, et al. Association of plasma beta-amyloid level and cognitive reserve with subsequent cognitive decline. JAMA 305, 261-266 (2011).

38. Vollmers C, Sit RV, Weinstein JA, Dekker CL, Quake SR. Genetic measurement of memory B-cell recall using antibody repertoire sequencing. Proc Natl Acad Sci U S A 110, 13463-13468 (2013).

39. Sievers F, et al. Fast, scalable generation of high-quality protein multiple sequence alignments using Clustal Omega. Mol Syst Biol 7, 539 (2011).

40. Sievers F, Higgins DG. Clustal Omega for making accurate alignments of many protein sequences. Protein Sci 27, 135-145 (2018).

41. Lefranc MP. IMGT databases, web resources and tools for immunoglobulin and T cell receptor sequence analysis, http://imgt.cines.fr. Leukemia 17, 260-266 (2003).

42. Ye J, Ma N, Madden TL, Ostell JM. IgBLAST: an immunoglobulin variable domain sequence analysis tool. Nucleic Acids Res 41, W34-40 (2013).

43. Lefranc MP, et al. IMGT, the international ImMunoGeneTics information system. Nucleic Acids Res 37, D1006-1012 (2009).

\section{Acknowledgements}

We sincerely thank the subjects, staffs of hospitals, technical supporting team members, and volunteers for this project. This work was supported by grants from the Ministry of Health and Welfare to I.M-J (HI19C1132020019, HU20C0187). This work was also supported by grants from the Ministry of Health and Welfare to J-C.P (HI19C1339). This work was supported by grants from the National Research Foundation of Korea to S-H.H (NRF2019R1I1A1A01063525). This work was also supported by the Global Research 
Development Center Program through the NRF funded by the Ministry of Science and ICT (MSIT) (2015K1A4A3047345), the Brain Korea 21 Plus Project in 2020, and the Ministry of Science and ICT(MSIT) of the Republic of Korea and the National Research Foundation of Korea (NRF-2020R1A3B3079653) to S.K.

\section{Author contributions}

J-C.P, J.N, S.J, S-H.H, S.K, and I.M-J conceptualized this study. J-C.P, J.N, K.K, H.C. D.L, D.Y, and A.C.L carried out experiments. J-C.P, J.N, S.J, H.L, and D.Y analyzed statistics. J.N, Y.L, and H.L analyzed the NGS data. J.C and DY.L provided the resources. J-C.P, J.N, S.J, and S-H.H wrote the original draft. S.K and I.M-J reviewed and edited the manuscript.

\section{Completing interests}

The authors declare no competing interests.

\section{Additional information}

Supplementary information is available for this paper at online. 


\section{Figures and Tables}

A

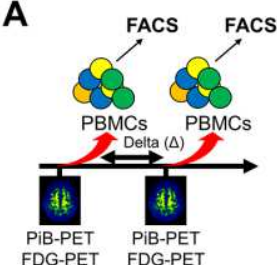

Baseline 2y Follow-up $(n=133)$

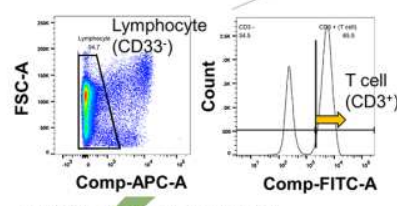
Multiple regression analysis

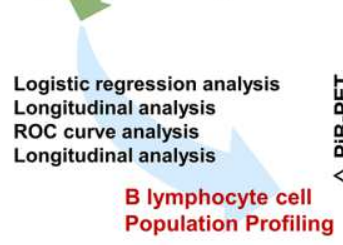

FACS sorting

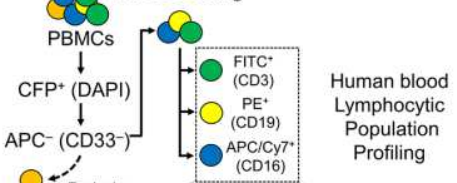

Mros

Myeloid (CD33+)

Profiling
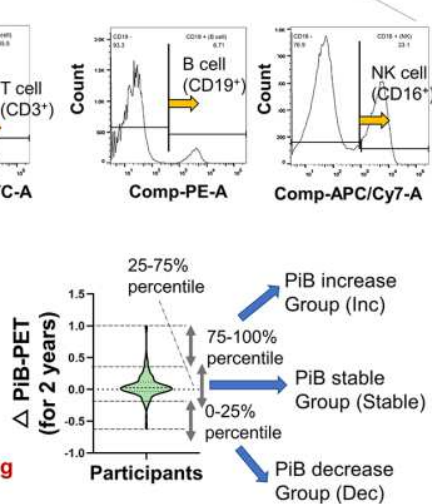

B

\begin{tabular}{|c|c|c|c|c|c|c|}
\hline \multicolumn{5}{|c|}{$\begin{array}{l}\text { Dependent variable: } \Delta \text { Global PiB } \\
\text { deposition (SUVR) }\end{array}$} & \multicolumn{2}{|c|}{$\begin{array}{l}\text { Method: } \\
\text { Stepwise }\end{array}$} \\
\hline $\begin{array}{l}\text { Indep. } \\
\text { variable }\end{array}$ & $\beta$ & SE & $t$ & $\begin{array}{c}P \\
\text { value }\end{array}$ & $\begin{array}{l}\text { F-ratio } \\
\text { (DF) }\end{array}$ & $\begin{array}{l}\text { Adj- } \\
\mathrm{R}^{2}\end{array}$ \\
\hline$\Delta \mathrm{B}$ cell & 0.0065 & 0.0017 & 3.698 & $0.0003^{\star \star \star}$ & $\begin{array}{c}13.67 \\
(1,131)\end{array}$ & 0.088 \\
\hline
\end{tabular}

C

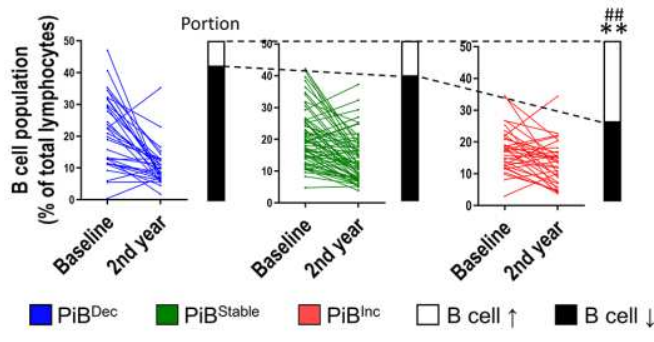

Figure 1. The strategy for longitudinal analysis (A) Timeline of the longitudinal study ( $\mathrm{n}=$ 133). Brain imaging and isolation of peripheral mononuclear cell (PBMC) were performed at both baseline (BL) and $2^{\text {nd }}$ year follow-up (FL) time-point. Classification of the PBMCs by the FACS analysis $\left(\mathrm{CD}^{+}\right.$for $\mathrm{T}$ lymphocytes, $\mathrm{CD} 19^{+}$for $\mathrm{B}$ lymphocytes, and $\mathrm{CD} 16^{+}$for $\mathrm{NK}$ lymphocytes). Participants were classified into three groups for the in-depth analyses (PiB increase group, $\mathrm{PiB}^{\text {Inc }}$, getting worse for 2 years; $\mathrm{PiB}$ stable group, $\mathrm{PiB}^{\text {Stable }}$, none the worse for 2 years; $\mathrm{PiB}$ possible decrease group, $\mathrm{PiB}^{\mathrm{Dec}}$, relatively getting better for 2 years). (B) Step-wise multiple regression analysis between the delta $(\Delta)$ lymphocytes $(\mathrm{B}, \mathrm{T}, \mathrm{NK}$ lymphocytes) and $\Delta$ global $\mathrm{PiB}$ deposition (standardized uptake value ratio; SUVR). Age and gender were also included as covariates. Only B lymphocyte is selected by the stepwise method. (C) B lymphocyte changes between baseline and follow-up $2^{\text {nd }}$ year. PiB ${ }^{\text {Inc }}$ group has higher portion of $\mathrm{B}$ cell increased- participants than $\mathrm{PiB}^{\mathrm{Dec}}$ and $\mathrm{PiB}^{\text {Stable }}$ group (Chi-trend test, ${ }^{\# \#} \mathrm{p}<0.01$; Chi-squared test, ${ }^{* *} \mathrm{p}<0.01$ ). 
A

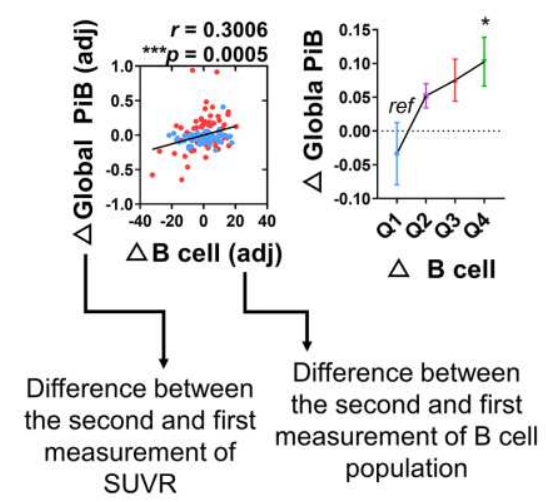

D

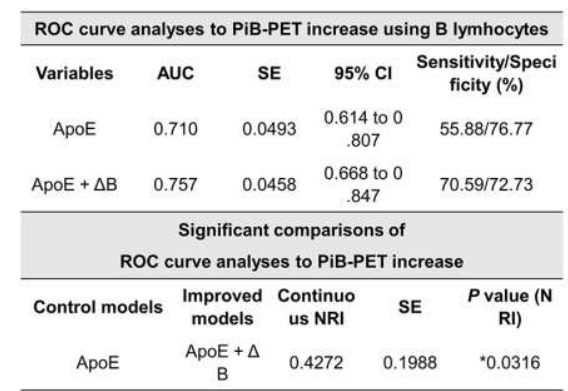

B

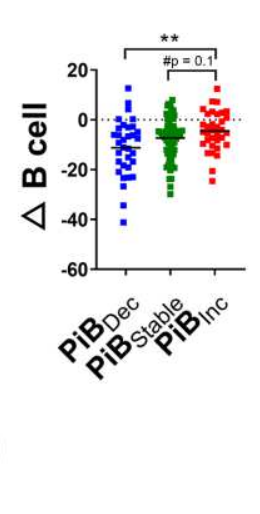

C
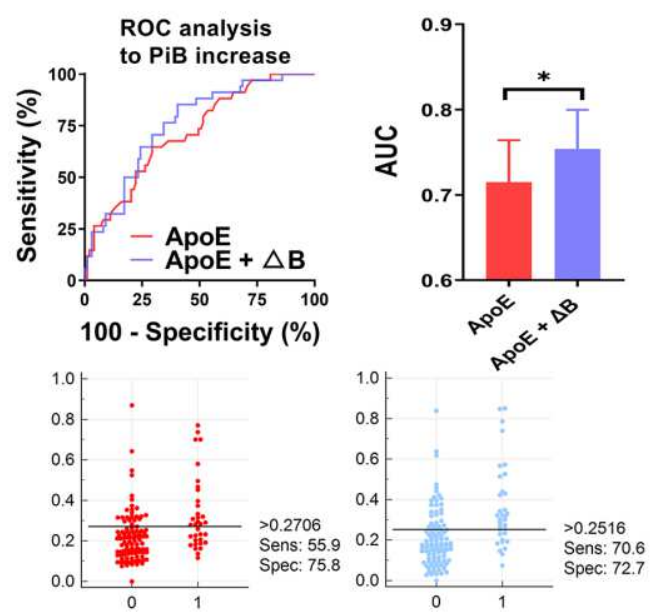

E

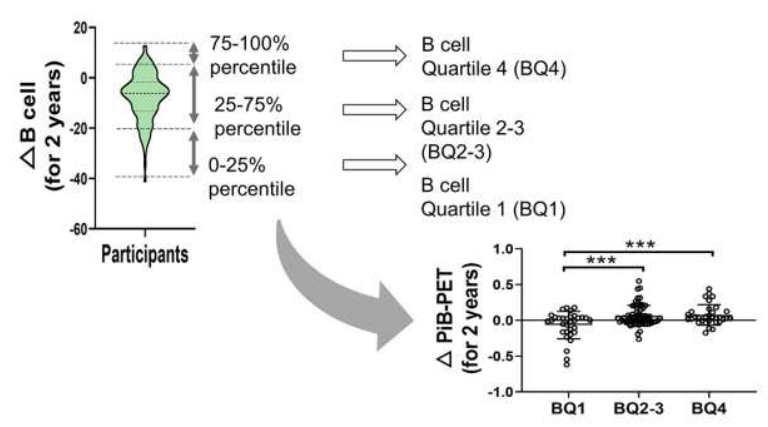

Figure 2. Longitudinal analysis and receiver operation curve (ROC) analysis for B lymphocyte profiling (A) Partial correlation analysis between $\Delta$ global PiB SUVR and $\Delta \mathrm{B}$ lymphocytes $(* * * p<0.001$, left) and ANOVA with post-hoc test between the quartiles of $\Delta$ B lymphocytes $(\mathrm{Q} 1<\mathrm{Q} 2<\mathrm{Q} 3<\mathrm{Q} 4)$ and $\Delta$ global PiB SUVR. (B) $\mathrm{PiB}^{\text {Inc }}$ group significantly showed less decrease in $\mathrm{B}$ cell population than $\mathrm{PiB}^{\mathrm{Dec}}$ group for 2 years ${ }^{* *} \mathrm{p}<0.01$, ANOVA with Tukey's post-hoc test). (C-D) Biomarkers of B cell showed better discrimination power to PiB-progression when combined with APOE genotyping. ${ }^{*} \mathrm{p}<0.05$ by continuous net reclassification index (NRI). Interactive dot diagram showed sensitivity/specificity/cut-off $\left(\mathrm{p}_{\mathrm{i}}\right.$ values by Youden index, $>0.2706,>0.2516$ ) values for PiB increase- ROC curves. (E) Participants were re-classified according to their quartiles of $\Delta \mathrm{B}$ lymphocytes $(\mathrm{BQ} 1<\mathrm{BQ} 2<\mathrm{BQ} 3<\mathrm{BQ} 4)$ and divided into three groups (BQ1, BQ2-3, BQ4). Delta PiB-PET values were significantly increased in BQ2-3 and BQ4 than BQ1 $(* * * p<0.001$; ANOVA with Tukey's post-hoc test). Two outliers were excluded by Grubb's test. 


\section{A}
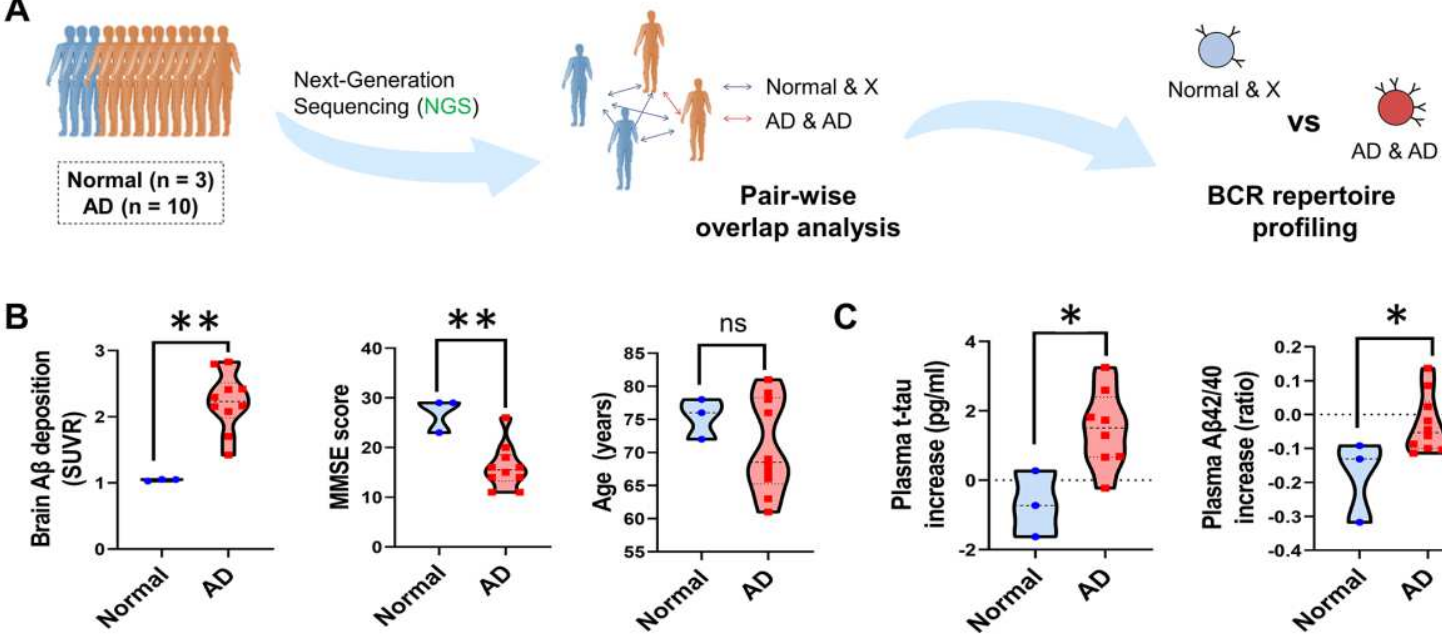

C
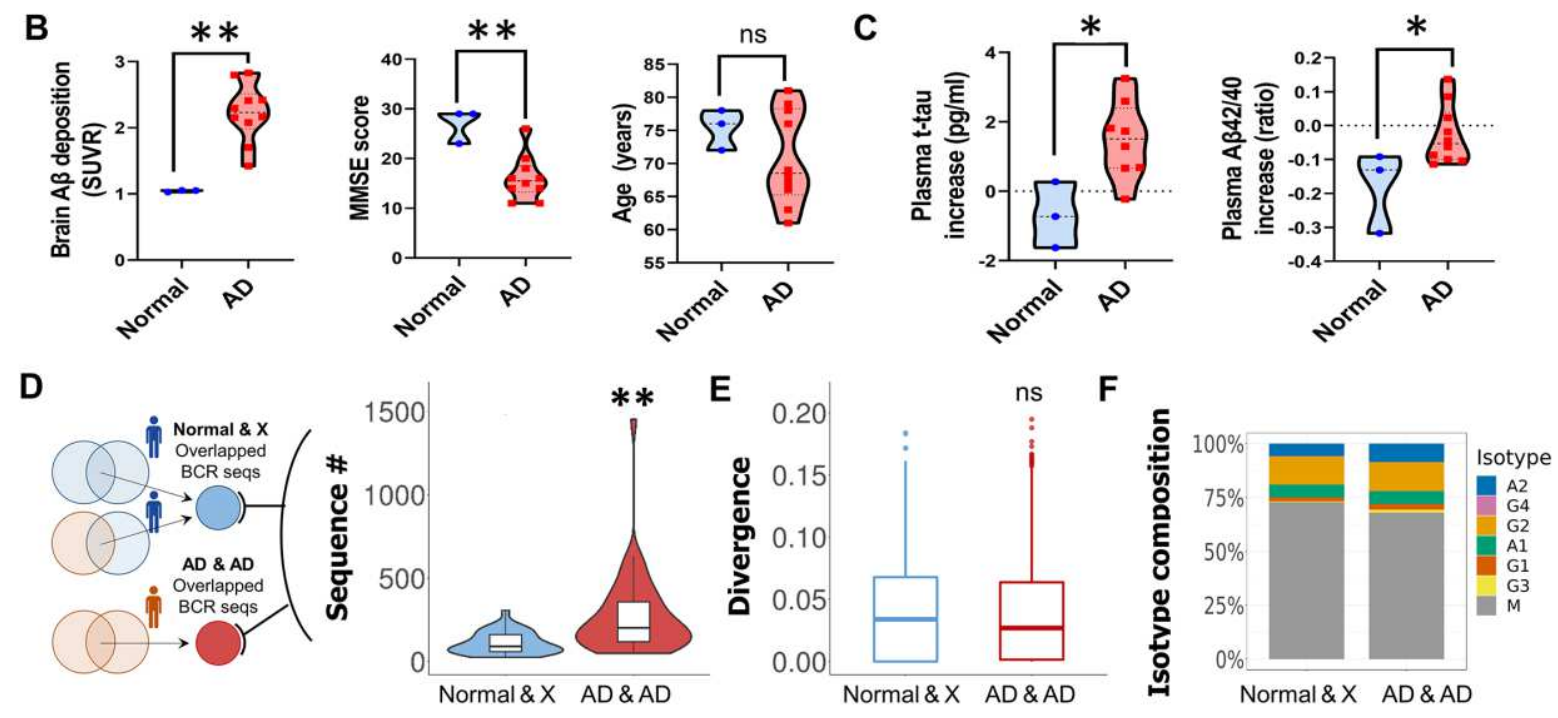

Figure 3. NGS-based BCR repertoire profiling followed by pair-wise BCR overlap analysis (A) B cell receptor $(B C R)$ repertoire analysis $(n=13)$. Total of 13 participants $(10$ $\mathrm{AD}$ patients and 3 cognitively normals) were used for the BCR repertoire analysis. Overlapped BCR sequences were defined by pair-wise overlap analysis on BCR repertoire of the samples, which was followed by comparative analysis between Normal\&X (comparison between Normal and Normal or Normal and AD; blue bidirectional arrow of the upper panel) and $\mathrm{AD \&} \mathrm{AD}$ (comparison between $\mathrm{AD}$ and $\mathrm{AD}$; red bidirectional arrow of the upper panel) groups. (B) Characterization of samples subjected to BCR repertoire analysis. ${ }^{*} \mathrm{p}<0.05$ and $* * \mathrm{p}<0.01$, independent $t$-test. (C) The changes in the levels of plasma t-tau and betaamyloid $42 / 40$ ratio. ${ }^{*} \mathrm{p}<0.05$, independent $t$-test. (D) The number of overlapped BCR sequences resulted from pair-wise overlap analysis. AD\&AD had more overlapped BCR sequences than Normal\&X. ${ }^{* *} p<0.001$; Wilcoxon rank-sum test. (E) Divergence of the overlapped BCR sequences. (F) Isotype composition of the overlapped BCR sequences. 

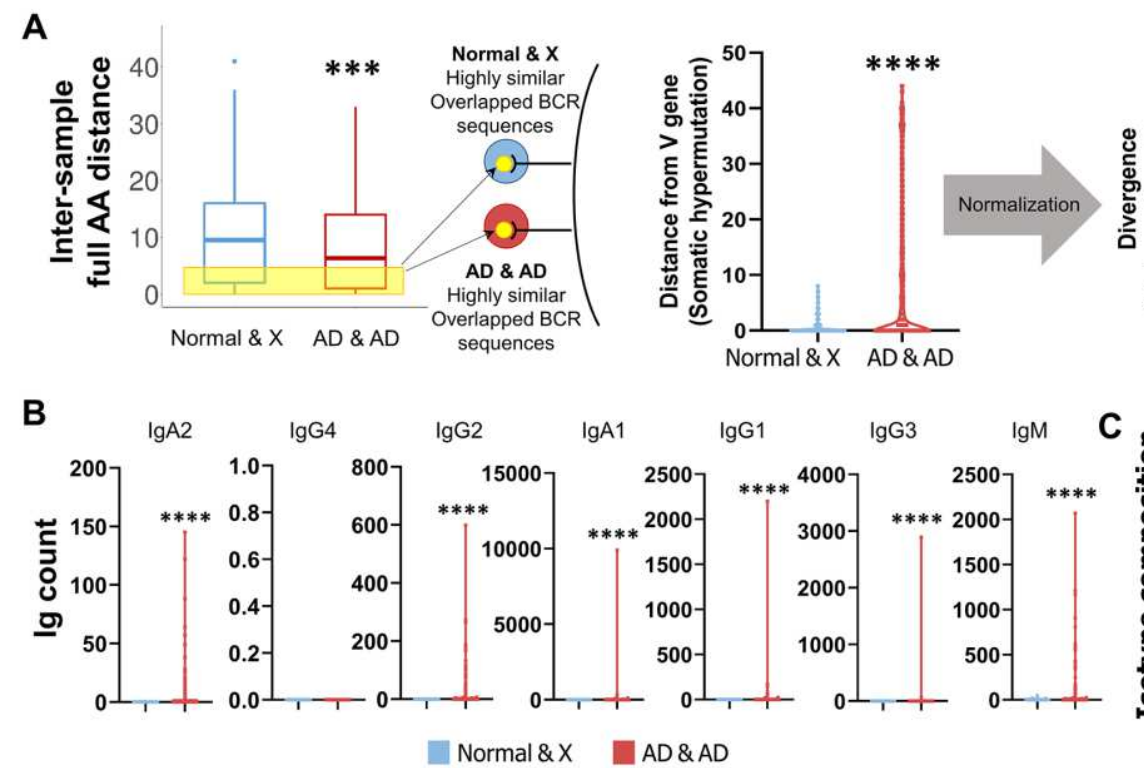

D

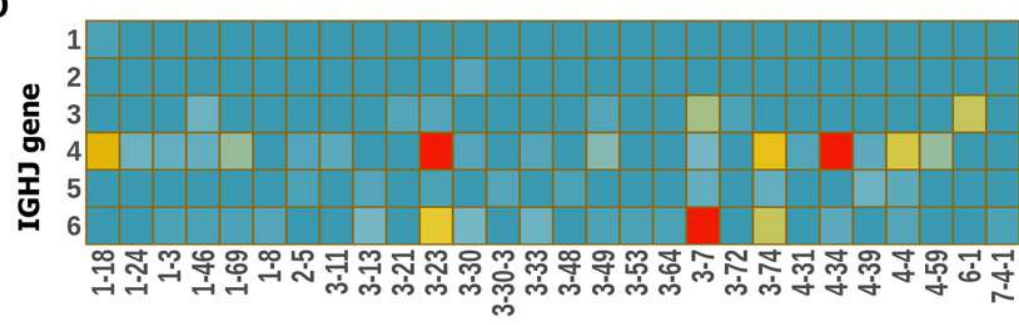

IGHV gene
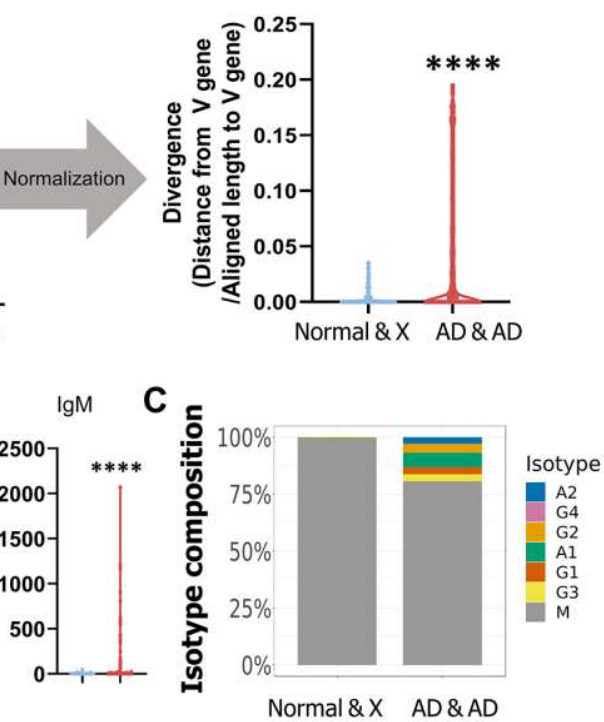

$\mathbf{E}$

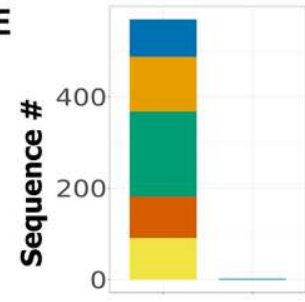

Identical Different Isotype identity

Figure 4. NGS-based BCR repertoire profiling followed by pair-wise BCR overlap analysis (high similar overlapped sequences) (A) Inter-sample full amino acid (AA) distance of the overlapped BCR sequences. The averaged hamming distance between BCR sequences, which were overlapped and derived from different samples, was calculated using full AA sequences. This distance was named as inter-sample full AA distance. Among the overlapped BCR sequences, sequences with inter-sample full AA distance value smaller than 5 were defined as 'highly similar overlapped BCR sequence'. Significantly increased divergence of highly similar overlapped BCR sequences in AD\&AD group. ****p $<0.0001$; Wilcoxon rank-sum test. (B) The number of Ig counts between Normal\&X vs AD\&AD. $* * * * \mathrm{p}<0.0001$; Man-Whitney test. (C) Isotype composition of the highly similar overlapped BCR sequences. Despite of a small inter-sample full AA distance, the sequences with a high divergence were only shown in AD\&AD. (D) VJ gene usage of the class-switched and highly similar overlapped BCR sequences. (E) Isotype identity of the class-switched and highly similar overlapped BCR sequences. Matches in isotypes in different samples of the classswitched and highly similar overlapped BCR sequences were quantified. If the isotypes of sequences were identical in different two samples, they were counted as "identical". And, if not, they were counted as "different". The isotype of the sequences was also represented by colors following the color coding used in (C). 
A

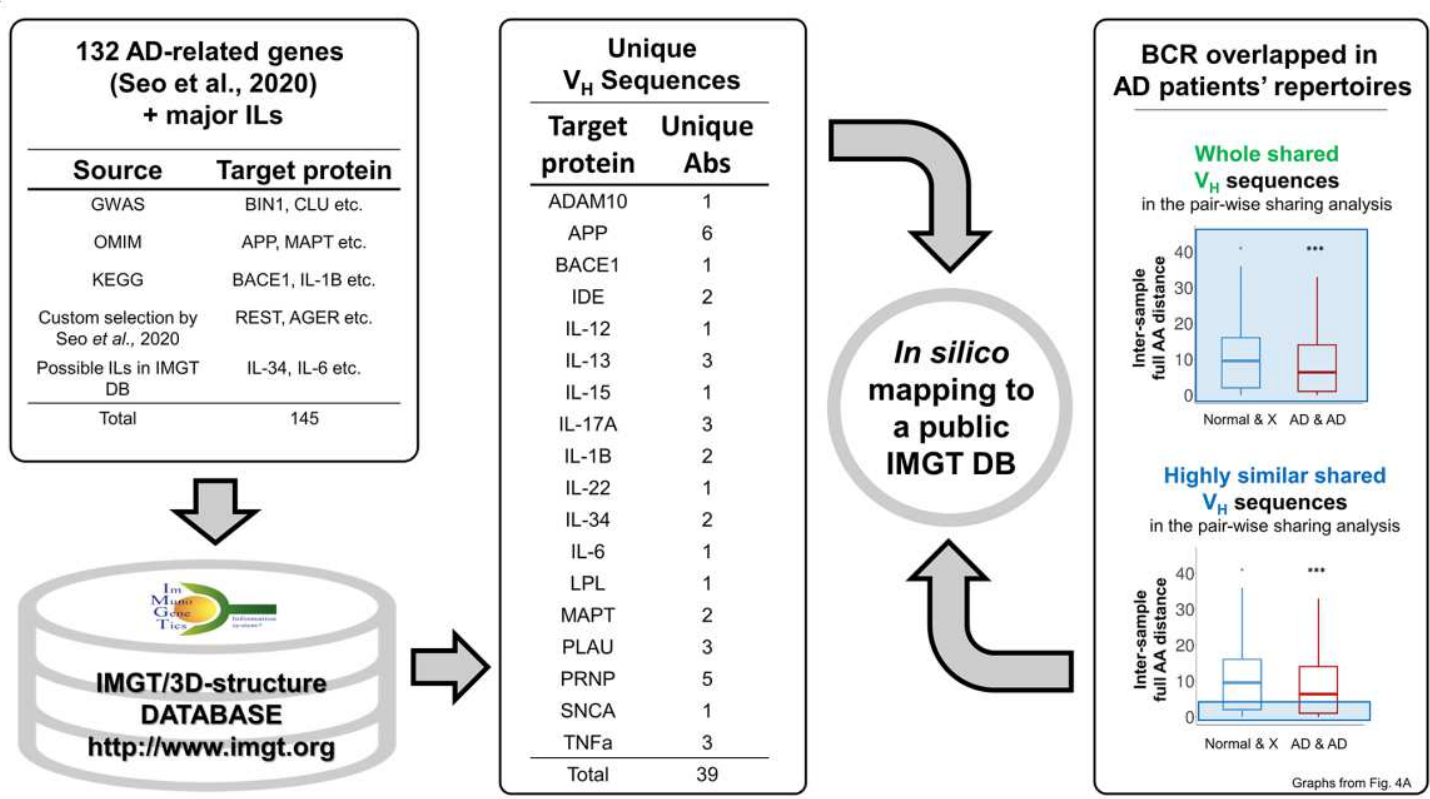

B

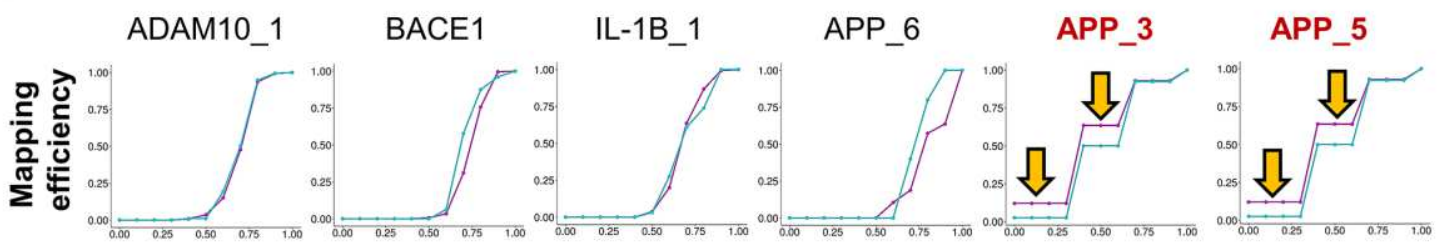

CDR3 AA mismatch threshold for whole shared $V_{H}$ sequences

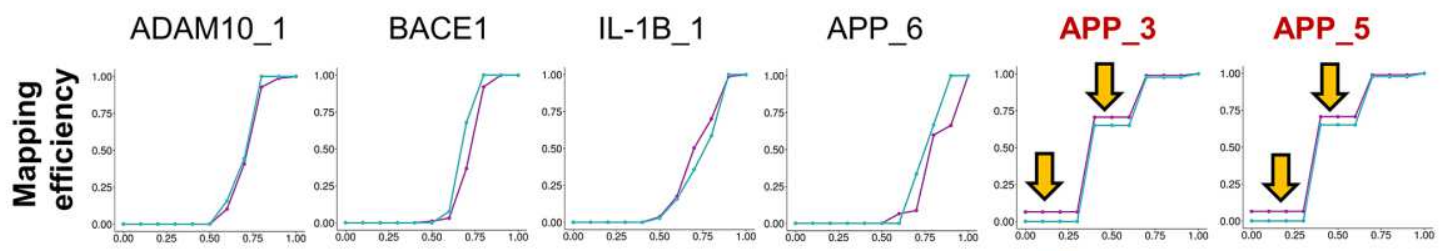

CDR3 AA mismatch threshold for highly similar shared $\mathrm{V}_{\mathrm{H}}$ sequences

C

- AD \& AD - Normal \& $X$

\begin{tabular}{|l|l|l|l|l|l|r|}
\hline seq_id & target_protein & CDR1_AA & CDR2_AA & CDR3_AA & V_gene & divergence \\
\hline APP_3 & APP & GFTFSRYS & INSVGSST & GDY & IGHV3-23*05 & 11.34 \\
\hline APP_5 & APP & GFTFSRYS & INSVGNST & GDY & IGHV3-23*04 & 12.37 \\
\hline
\end{tabular}

APP-specific VH sequence from IMGT database

Figure 5. In silico mapping of the overlapped BCR sequences to antibodies binding to AD-related genes (A) Experimental flow chart for In silico mapping analysis. The Immunogenetics information system (IMGT) database was used for the generation of antibody sequences (unique $\mathrm{V}_{\mathrm{H}}$ sequences). (B) The mapping results from the comparison between the whole or highly shared $\mathrm{V}_{\mathrm{H}}$ sequences and the unique $\mathrm{V}_{\mathrm{H}}$ sequences. The APPbinding antibodies showed different mapping patterns between AD\&AD and Normal\&X. (C) The information of APP-specific $\mathrm{V}_{\mathrm{H}}$ sequences from the IMGT database. 


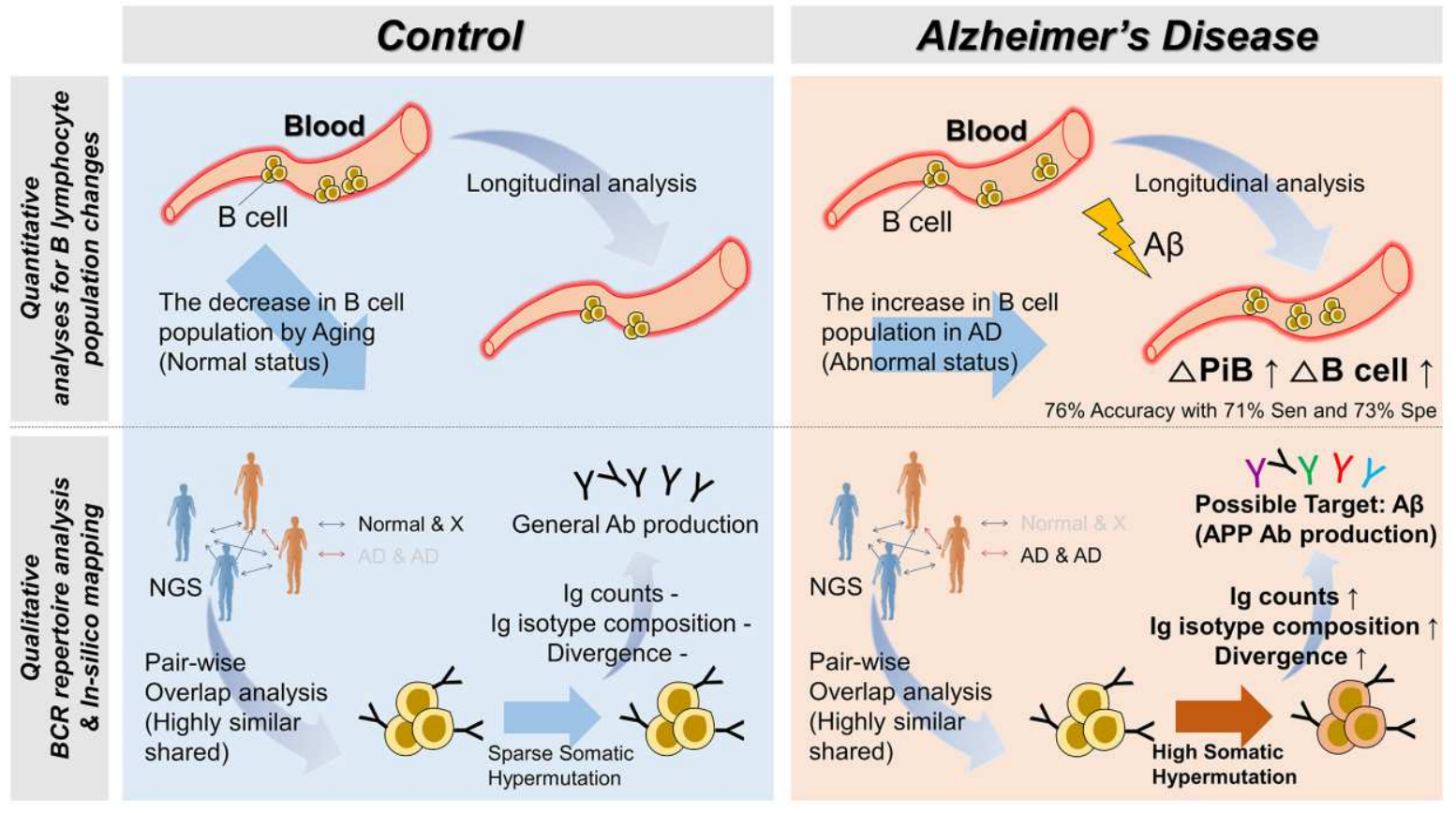

Figure 6. A graphical summary of this study From the quantitative and longitudinal analysis, we found that the population change in B lymphocyte over two years is associated with the increase in cerebral beta-amyloid deposition in AD. Furthermore, from the qualitative BCR repertoire analysis and in-silico mapping results, we identified that the commonalities of BCR repertoires within AD patients (Ig counts, Ig isotype composition, divergence) and suggested the possibility of the immunological reaction to amyloid precursor protein (APP). NGS, next-generation sequencing; Ab, antibody; A $\beta$, beta-amyloid; APP, amyloid precursor protein; Ig, immunoglobulin. 
Table 1. Demographic information and details of ROC curve analysis

\begin{tabular}{lrrrrr}
\hline Characteristics & $\mathbf{P i B}^{\text {Dec }} \mathbf{( 3 3 )}$ & $\mathbf{P i B}^{\text {Stable }}(\mathbf{6 6})$ & $\mathbf{P i B}^{\text {Inc }}(\mathbf{3 4})$ & \multicolumn{1}{c}{ P-value } \\
\hline Gender, M/F (n) & $14 / 19$ & $30 / 36$ & $15 / 19$ & $\mathbf{P}=0.94$
\end{tabular}

\begin{tabular}{lcccc}
\hline Age, years, mean \pm SEM & $73.97 \pm 1.46$ & $67.51 \pm 0.83$ & $72.79 \pm 1.11$ & $<0.001^{*}$ \\
\hline Education, mean \pm SEM & $11.67 \pm 0.73$ & $11.27 \pm 0.63$ & $11.52 \pm 0.95$ & $\mathrm{P}=0.93$ \\
& & & & \\
\hline MMSE score, mean \pm SEM & $23.97 \pm 0.82$ & $25.83 \pm 0.47$ & $22.12 \pm 0.85$ & $<0.001^{*}$ \\
& & & & \\
\hline CN/MCI/Dementia & $18 / / 10 / 5$ & $53 / 9 / 4$ & $15 / 8 / 11$ & $<0.001^{\dagger}$
\end{tabular}

$\Delta \mathrm{PiB}$, mean \pm SEM $\quad-0.14 \pm 0.02 \quad 0.02 \pm 0.01 \quad 0.29 \pm 0.04 \quad<0.001^{*}$

$\Delta \mathbf{B}$, mean \pm SEM $\quad-11.20 \pm 2.01 \quad-7.31 \pm 1.06 \quad-4.48 \pm 1.36 \quad<0.05^{*}$

\begin{tabular}{lllll}
\hline ApoE4 positivity, e4+/N (\%) & $14 / 33(42.4 \%)$ & $11 / 66(15.7 \%)$ & $14 / 34(41.2 \%)$ & $<0.01^{\dagger}$
\end{tabular}

Abbreviations: $\mathrm{CN}$, cognitively normal; $\mathrm{MCI}$, mild cognitive impairment; ADD, Alzheimer's disease dementia; PiB, Pittsburgh compound B; SEM, Standard Error of Mean; n, number of participants; MMSE, Mini-Mental State Examination; ApoE, Apolipoprotein E; SUVR, standardized uptake value ratio; N, total number of participants. $\Delta \mathrm{PiB}$, the changes in cerebral amyloid deposition for 2 years; $\Delta \mathrm{B}$, the changes in $\mathrm{B}$ lymphocyte cell numbers for 2 years.

- *, significance by $t$-test; $\uparrow$, significance by chi-squared test. 


\section{Supplementary information}

\section{Title: Association of $B$ cell profile and receptor repertoire with the progression of}

Alzheimer's disease

Park et al.

\section{Supplementary information}

Supplementary Fig. 1: The gating strategy for analysis; PBMC T, B, and NK lymphocytes population

Supplementary Fig. 2: The increase in $\mathrm{B}$ cell in $\mathrm{PiB}^{\mathrm{Inc}}$ group

Supplementary Fig. 3: Isotype composition of BCR repertoire of the samples

Supplementary Fig. 4: Divergence of BCR repertoire of the samples

Supplementary Fig. 5: VJ gene usage of the whole BCR repertoire of the samples

Supplementary Fig. 6: VJ gene usage of the class-switched BCR repertoire of the samples

Supplementary Fig. 7: Phylogenetic analysis for the representative BCR lineages composed of the highly similar overlapped BCR sequences

Supplementary Fig. 8: In silico mapping results of 39 BCR sequences (whole sequences overlapped) binding to AD-related proteins

Supplementary Fig. 9: In silico mapping results of 39 BCR sequences (highly similar shared) binding to AD-related proteins

Supplementary Table 1: Reagents

Supplementary Table 2: Statistics for BCR repertoire NGS data

Supplementary Table 3: Class-switched and highly similar overlapped BCR lineages 

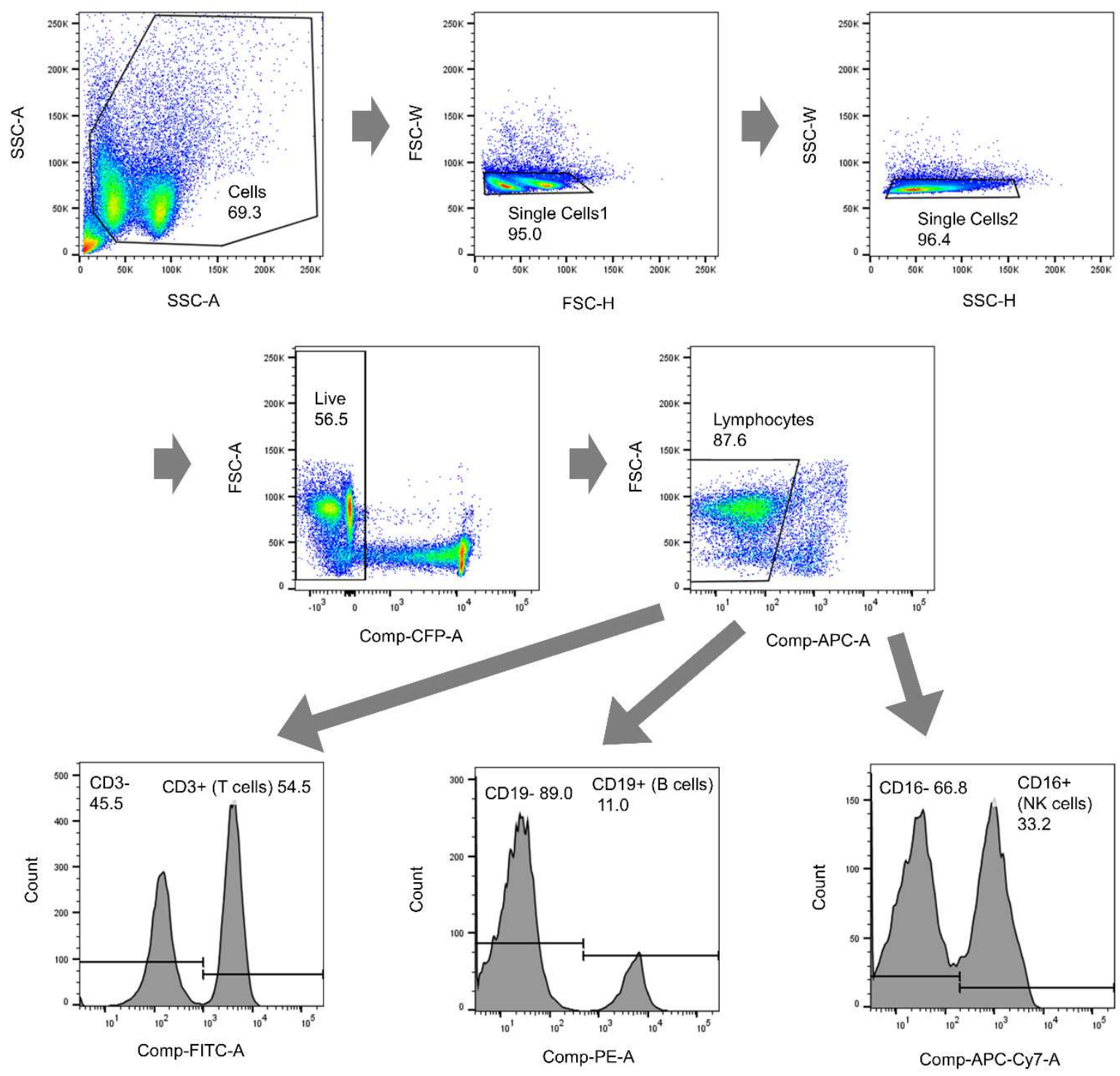

Supplementary Fig. 1. The gating strategy for analysis; PBMC T, B, and NK lymphocytes population Singlets were selected by forward scatter and side scatter. After excluding DAPI positive dead cells and pan-myeloid marker (CD33) positive population, remaining populations were identified as pan-T cell marker (CD3), pan-B cell marker (CD19), and pan-NK marker (CD16) positive populations. 
A PiB-

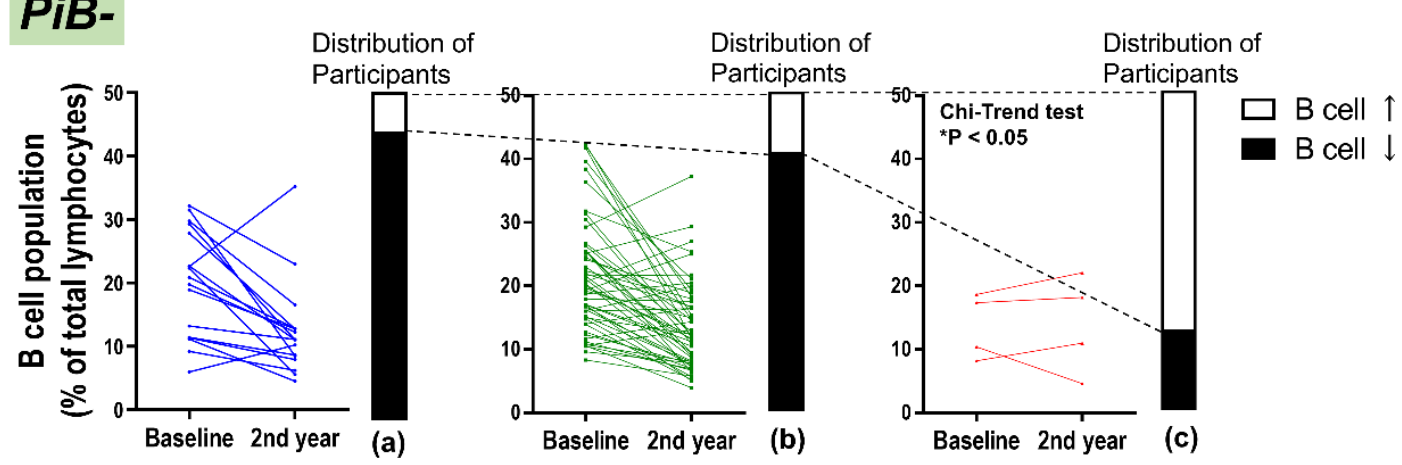

B

\section{PiB+}
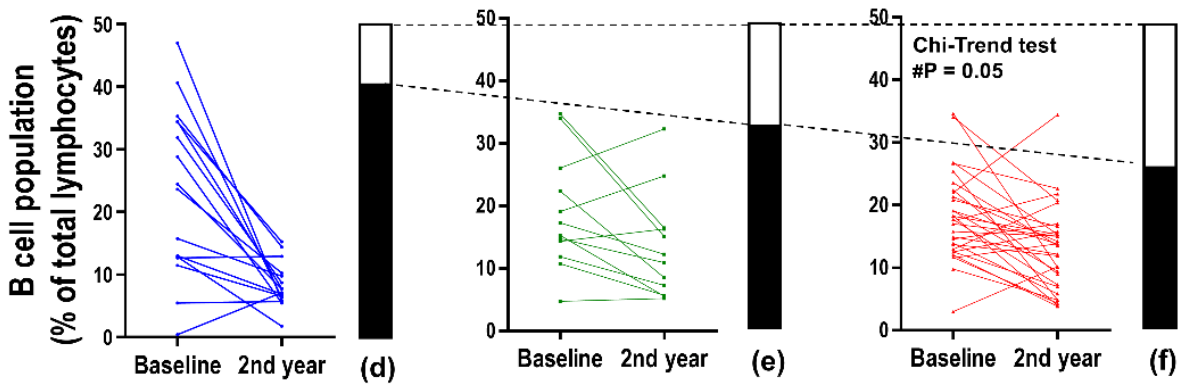

$\square$ B cell $\uparrow$

B cell $\downarrow$

C

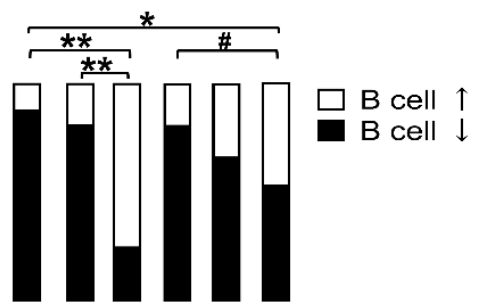

(a) (b) (c) (d) (e) (f)

Supplementary Fig. 2. The increase in $\mathrm{B}$ cell in $\mathrm{PiB}^{\mathrm{Inc}}$ group (A-B) $\mathrm{PiB}^{\mathrm{Inc}}$ group has a higher portion of $\mathrm{B}$ cell increased- participants than $\mathrm{PiB}^{\mathrm{Dec}}$ and $\mathrm{PiB}^{\text {Stable }}$ group both in $\mathrm{PiB}-$ and $\mathrm{PiB}+$.

(C) Chi-squared test $\left({ }^{\#} \mathrm{p}<0.10,{ }^{*} \mathrm{p}<0.05,{ }^{* *} \mathrm{p}<0.01\right)$. 


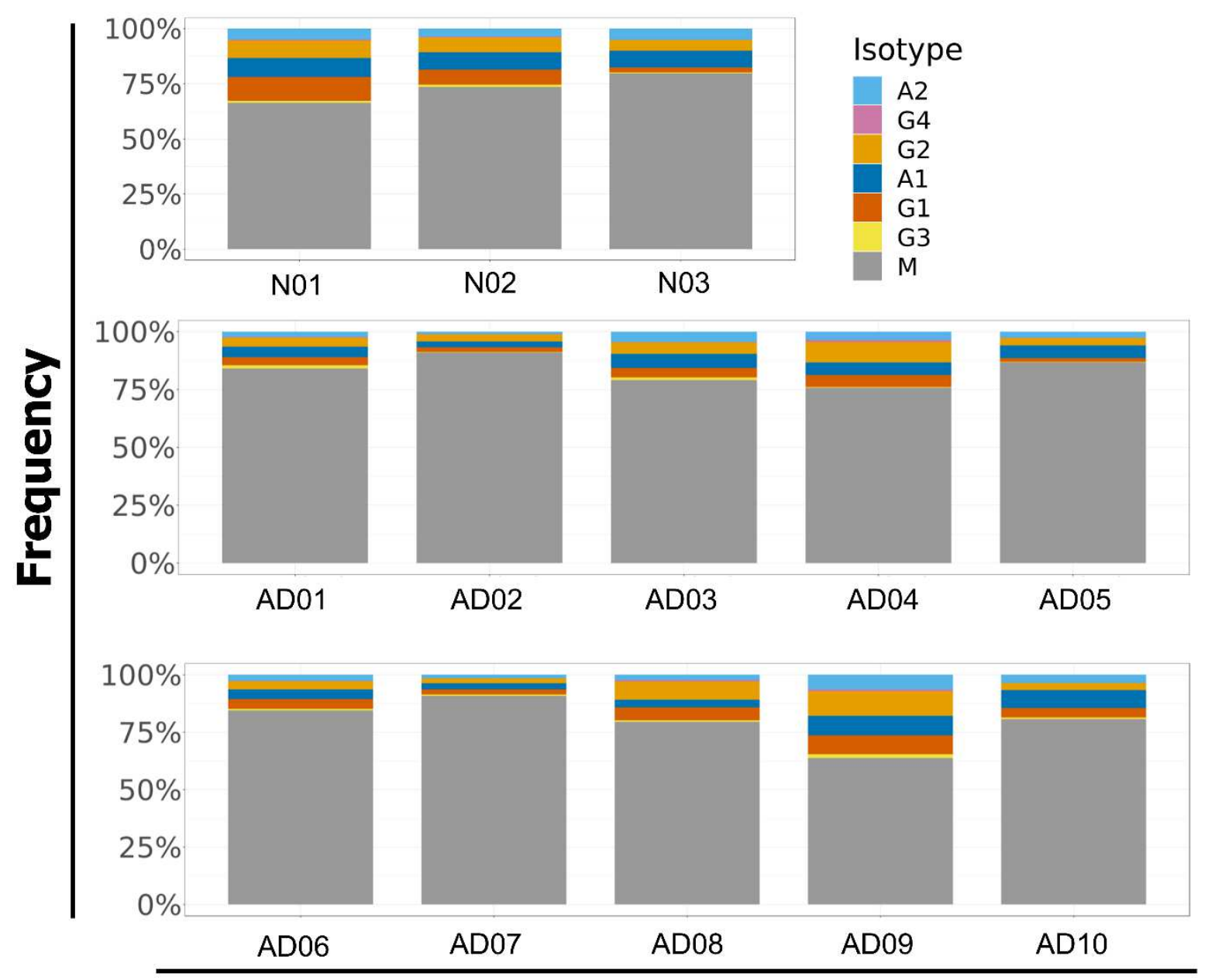

40

Supplementary Fig. 3. Isotype composition of BCR repertoire of the samples Isotype composition of BCR repertoire was calculated for each sample. Values were calculated in the number of unique BCR sequences. 

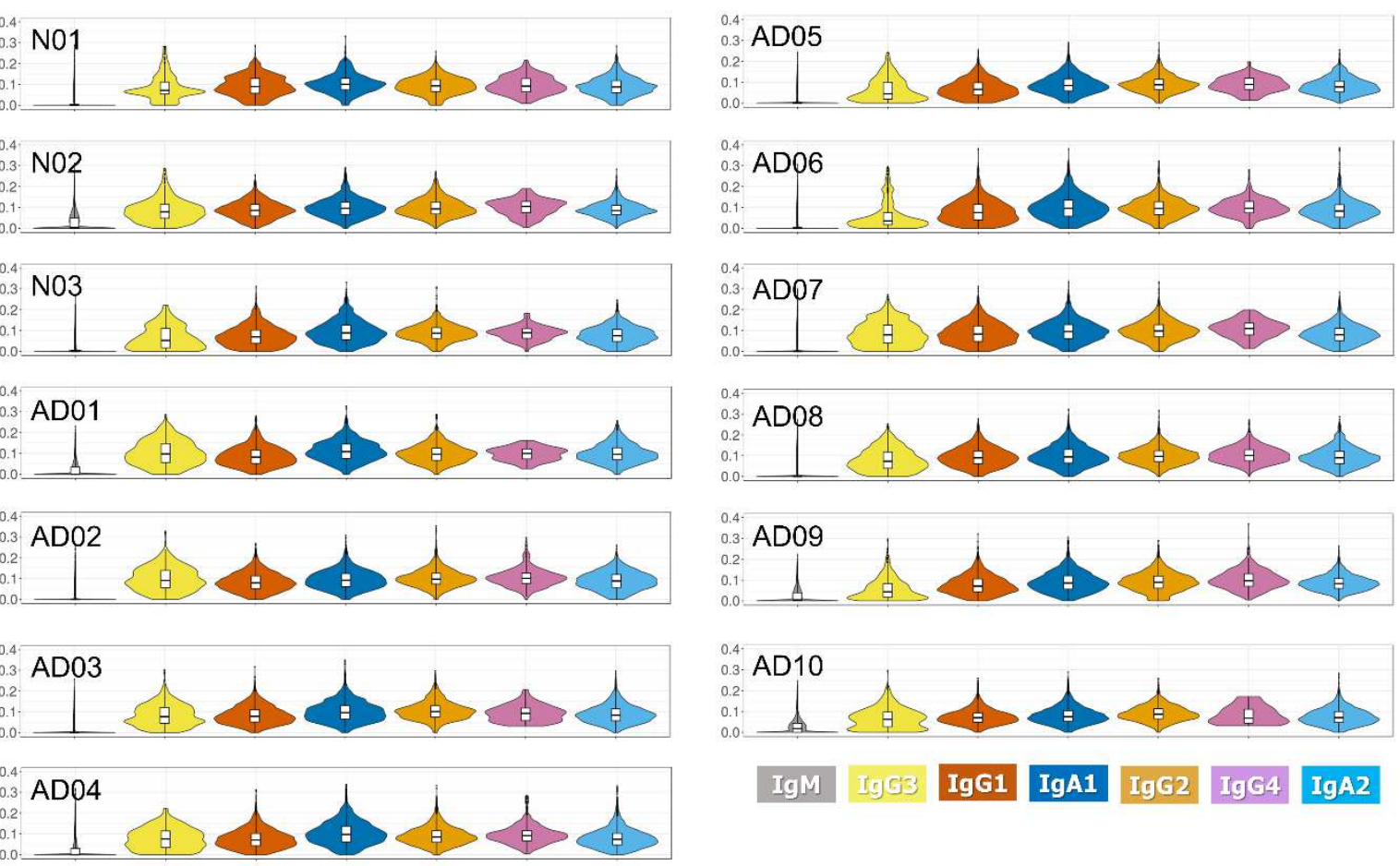

Supplementary Fig. 4. Divergence of BCR repertoire of the samples Divergence values of the BCR sequences were calculated by each isotype. Isotypes were allocated on the $\mathrm{x}$-axis according to the sequential order of class-switching events. Sample names were represented on the left top of the violin plots. 


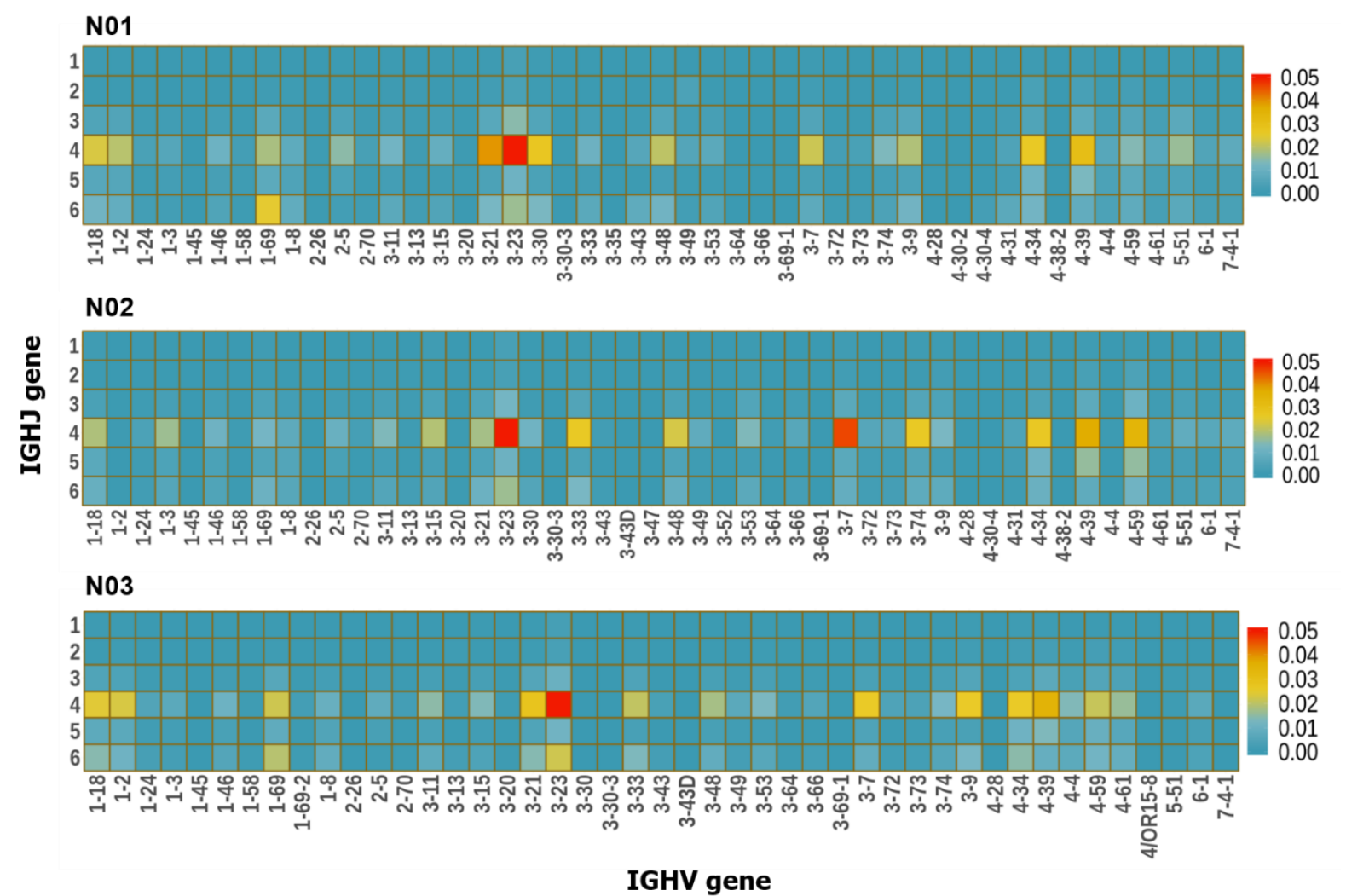

Supplementary Fig. 5-1. VJ gene usage of the whole BCR repertoire of the samples (Normal \#1 to Normal \#3) VJ gene usage of BCR repertoire of the sample was calculated in the number of unique BCR sequences and displayed as a heatmap. To minimize the effect of outliers, a threshold value of 0.05 was used. Sample names were represented on the left top of 57 the heatmaps. 
AD01

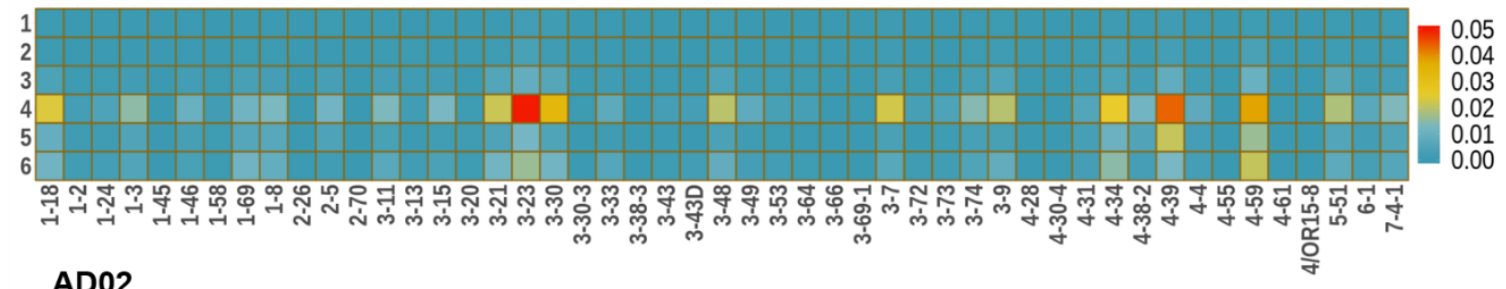

AD02
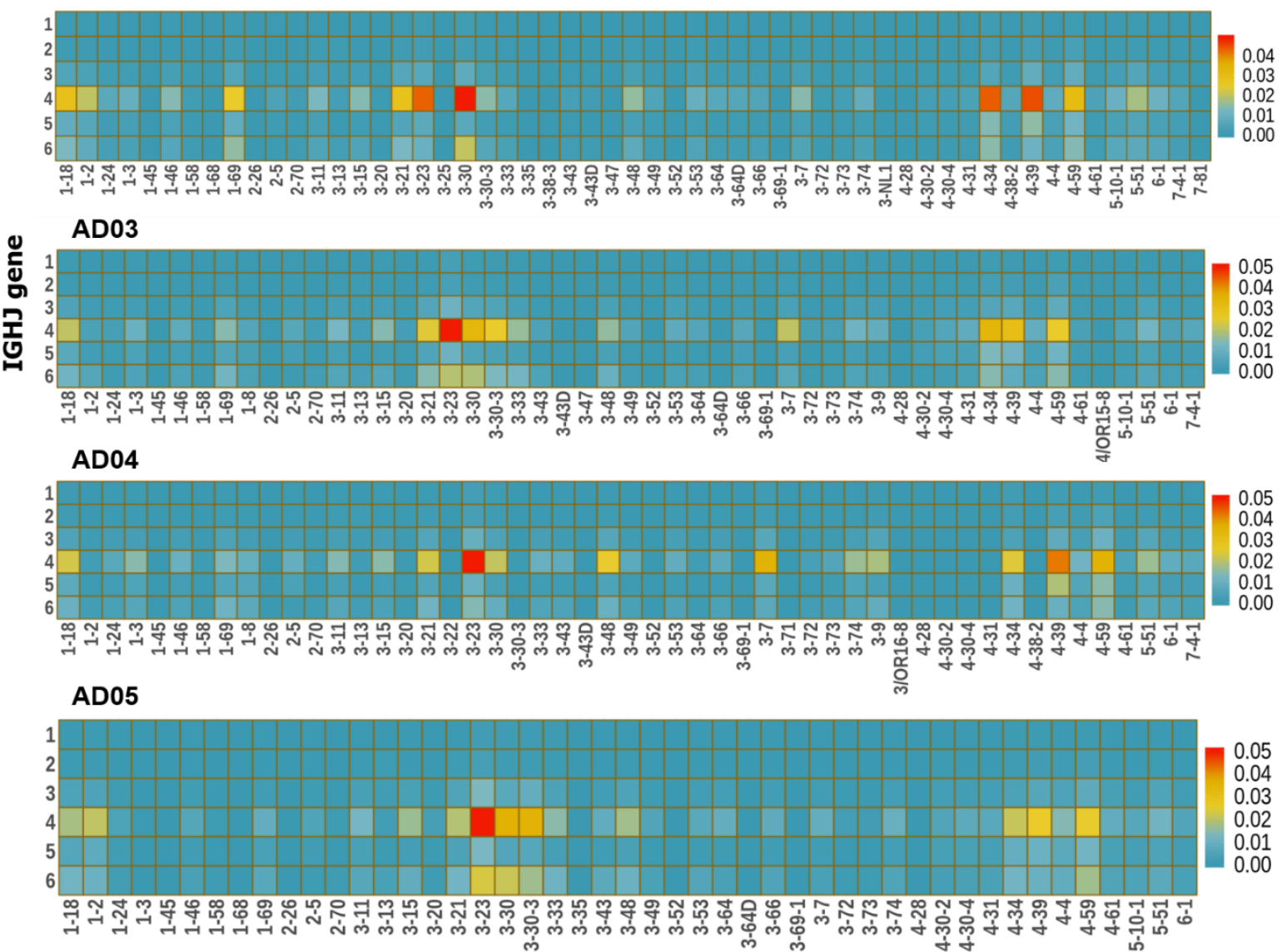

IGHV gene

60 Supplementary Fig. 5-2. VJ gene usage of the whole BCR repertoire of the samples (AD

61 \#1 to AD \#5) VJ gene usage of BCR repertoire of the sample was calculated in the number of 62 unique BCR sequences and displayed as a heatmap. To minimize the effect of outliers, a 63 threshold value of 0.05 for visualization was used. Sample names were represented on the left 64 top of the heatmaps. 
AD06

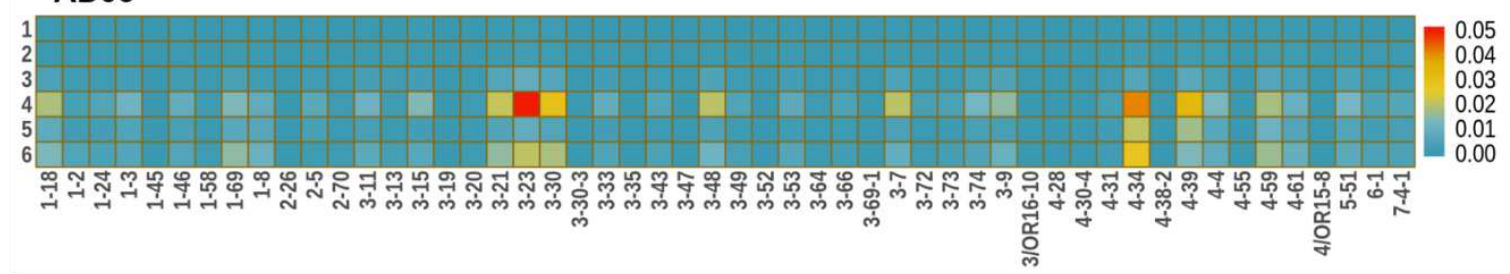

AD07
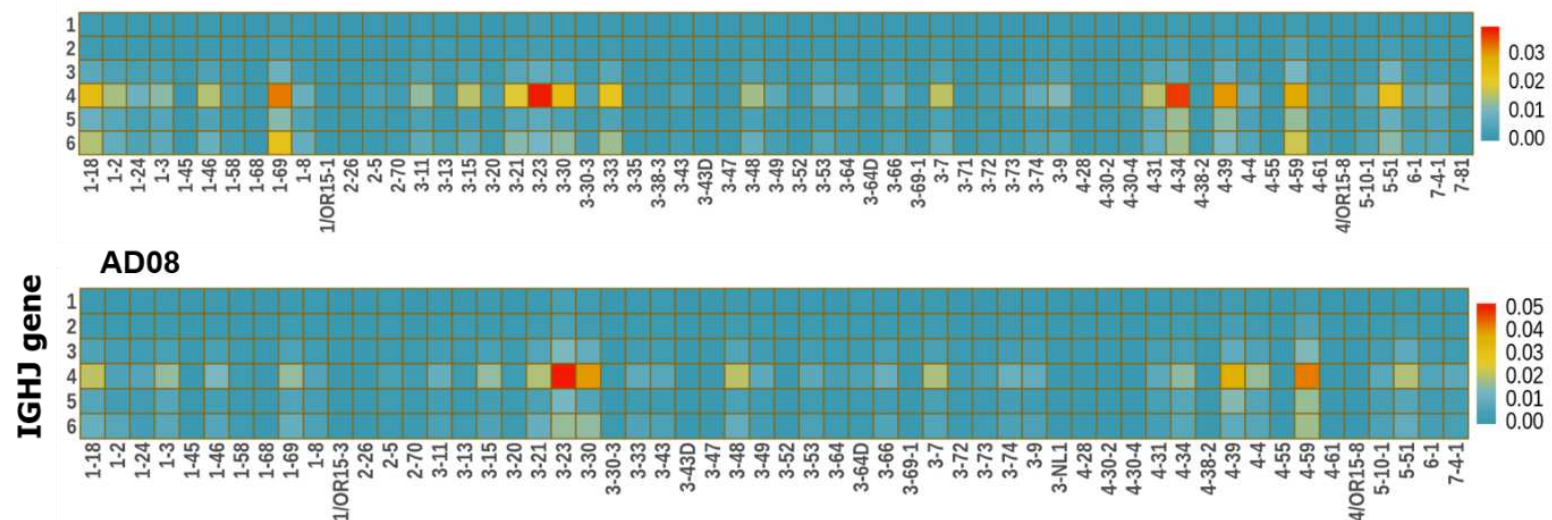

AD09

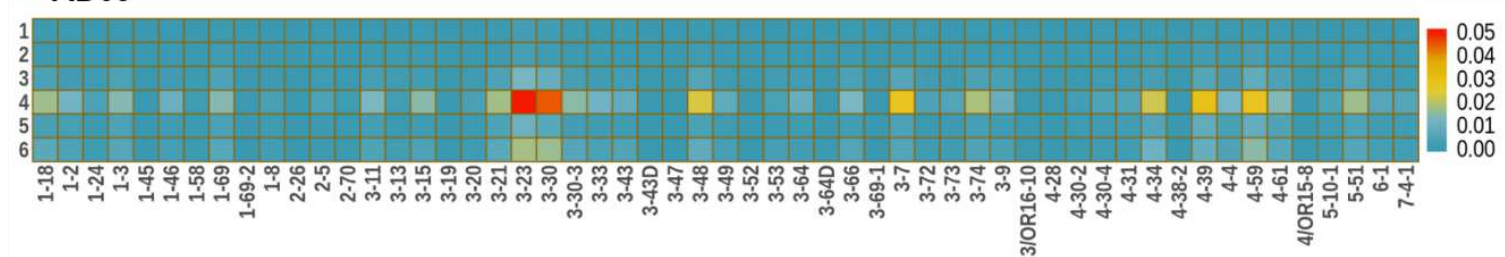

AD10

66

67

68

69

70

71

72

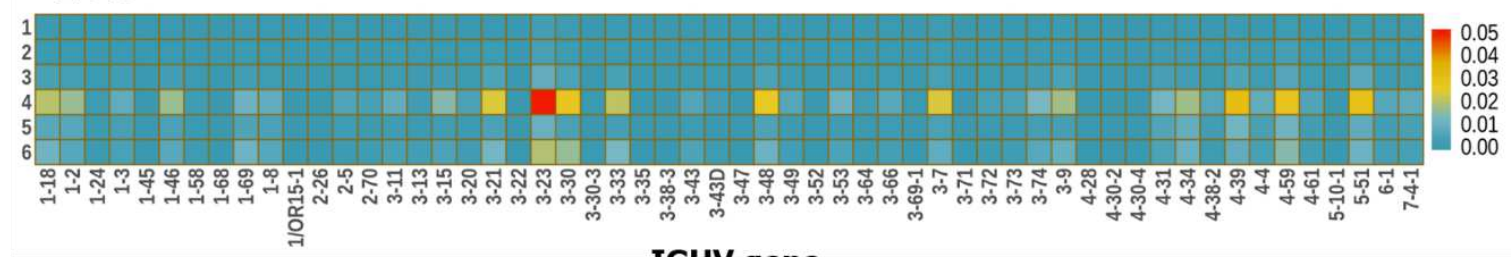

IGHV gene

Supplementary Fig. 5-3. VJ gene usage of the whole BCR repertoire of the samples (AD \#6 to AD \#10) VJ gene usage of BCR repertoire of the sample was calculated in the number of unique BCR sequences and displayed as a heatmap. To minimize the effect of outliers, a threshold value of 0.05 was used. Sample names were represented on the left top of the heatmaps. 


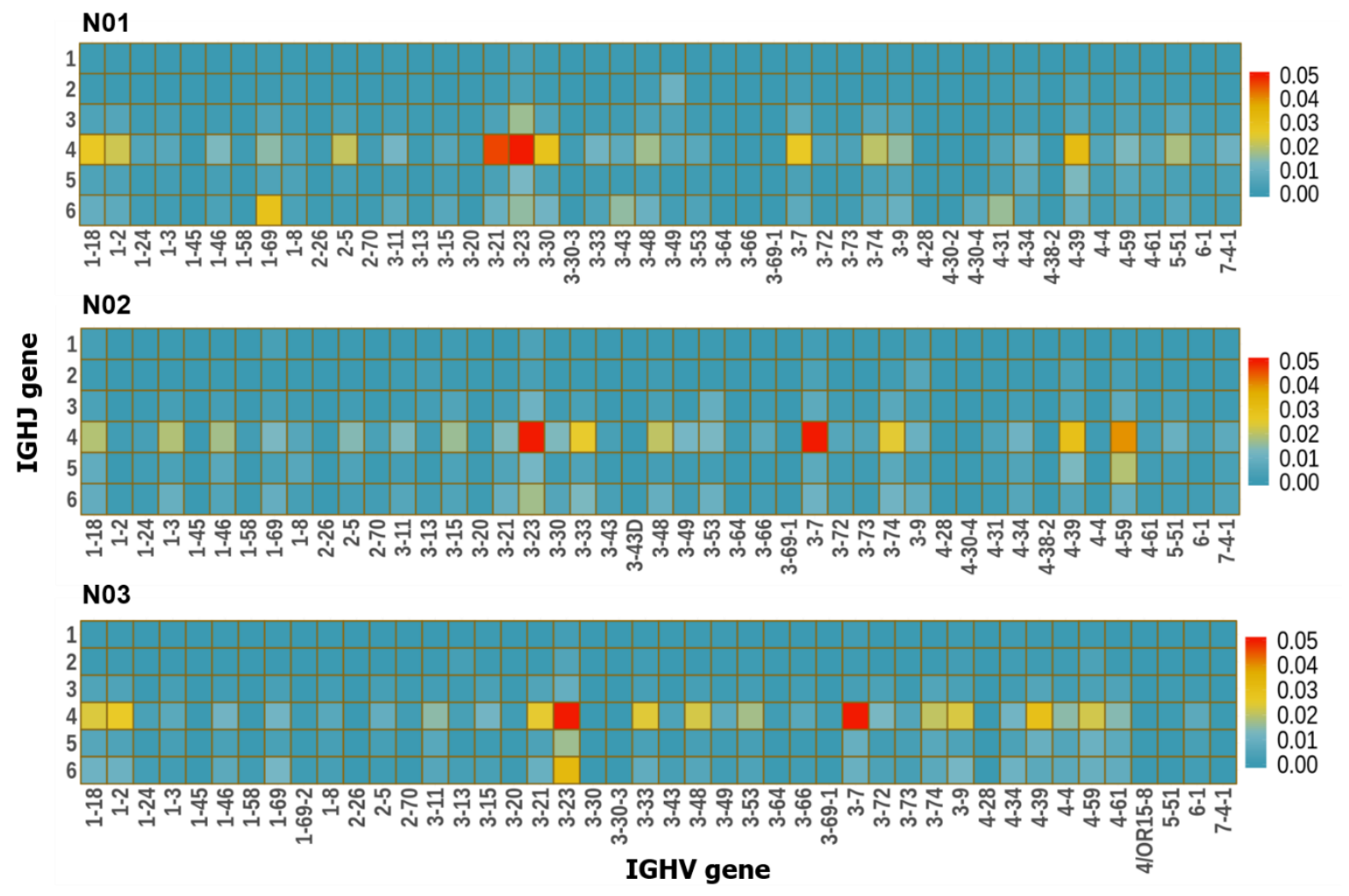

Supplementary Fig. 6-1. VJ gene usage of the class-switched BCR repertoire of the samples (Normal \#1 to Normal \#3) VJ gene usage of the class-switched BCR repertoire of the sample was calculated in the number of unique BCR sequences and displayed as a heatmap. The class-switched BCR repertoire means the collection of BCR sequences with isotypes other than IgM or IgD. Values were calculated within the defined class-switched population. To minimize the effect of outliers, a threshold value of 0.05 was used. Sample names were represented on the left top of the heatmaps. 


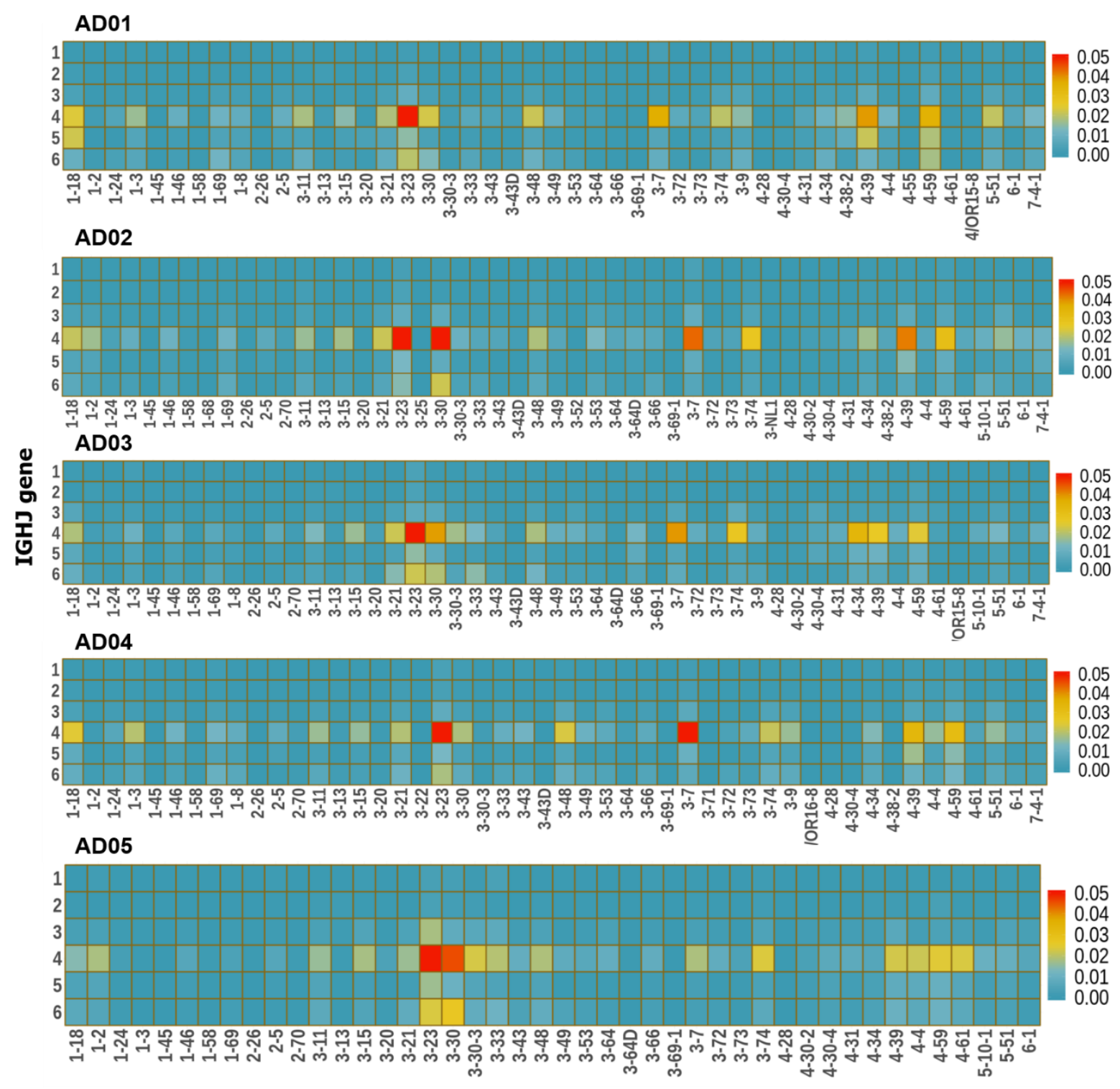

Supplementary Fig. 6-2. VJ gene usage of the class-switched BCR repertoire of the samples (AD \#1 to AD \#5) VJ gene usage of the class-switched BCR repertoire of the sample was calculated in the number of unique BCR sequences and displayed as a heatmap. The classswitched BCR repertoire means the collection of BCR sequences with isotypes other than IgM or IgD. Values were calculated within the defined class-switched population. To minimize the effect of outliers, a threshold value of 0.05 was used. Sample names were represented on the left top of the heatmaps. 


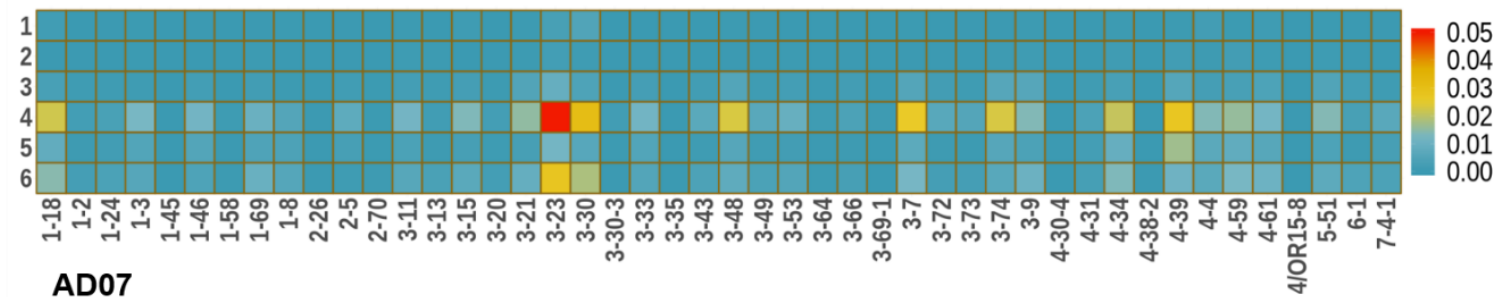

AD07
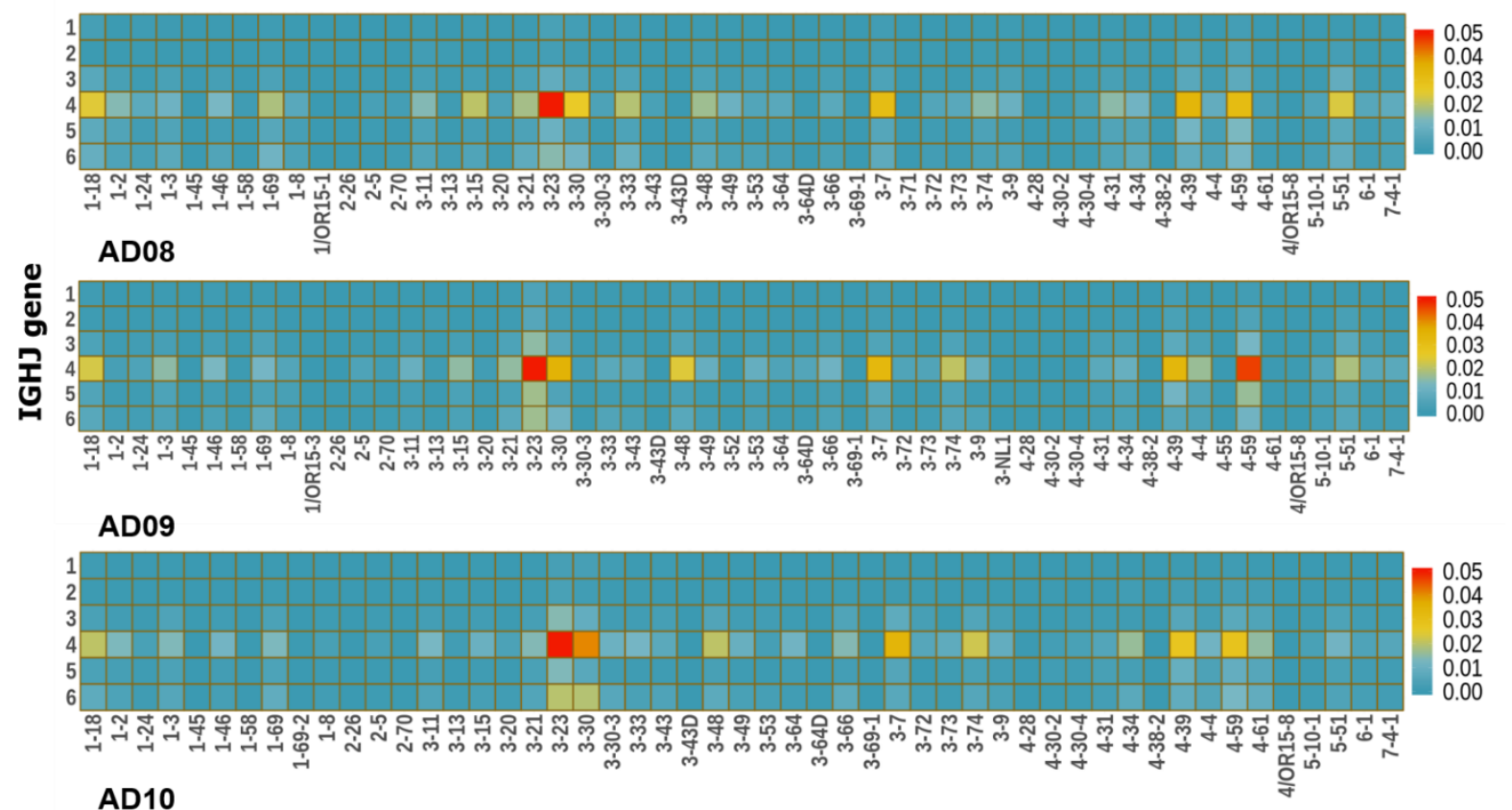

AD10

Supplementary Fig. 6-3. VJ gene usage of the class-switched BCR repertoire of the samples (AD \#6 to AD \#10) VJ gene usage of the class-switched BCR repertoire of the sample was calculated in the number of unique BCR sequences and displayed as a heatmap. The classswitched BCR repertoire means the collection of BCR sequences with isotypes other than IgM or IgD. Values were calculated within the defined class-switched population. To minimize the effect of outliers, a threshold value of 0.05 was used. Sample names were represented on the left top of the heatmaps. 
A
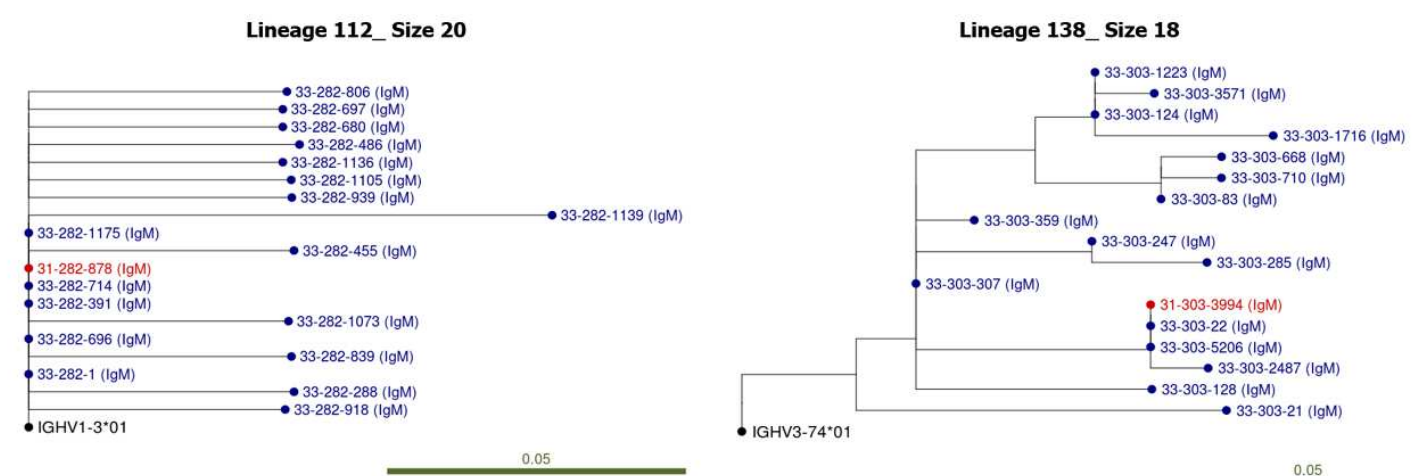

B
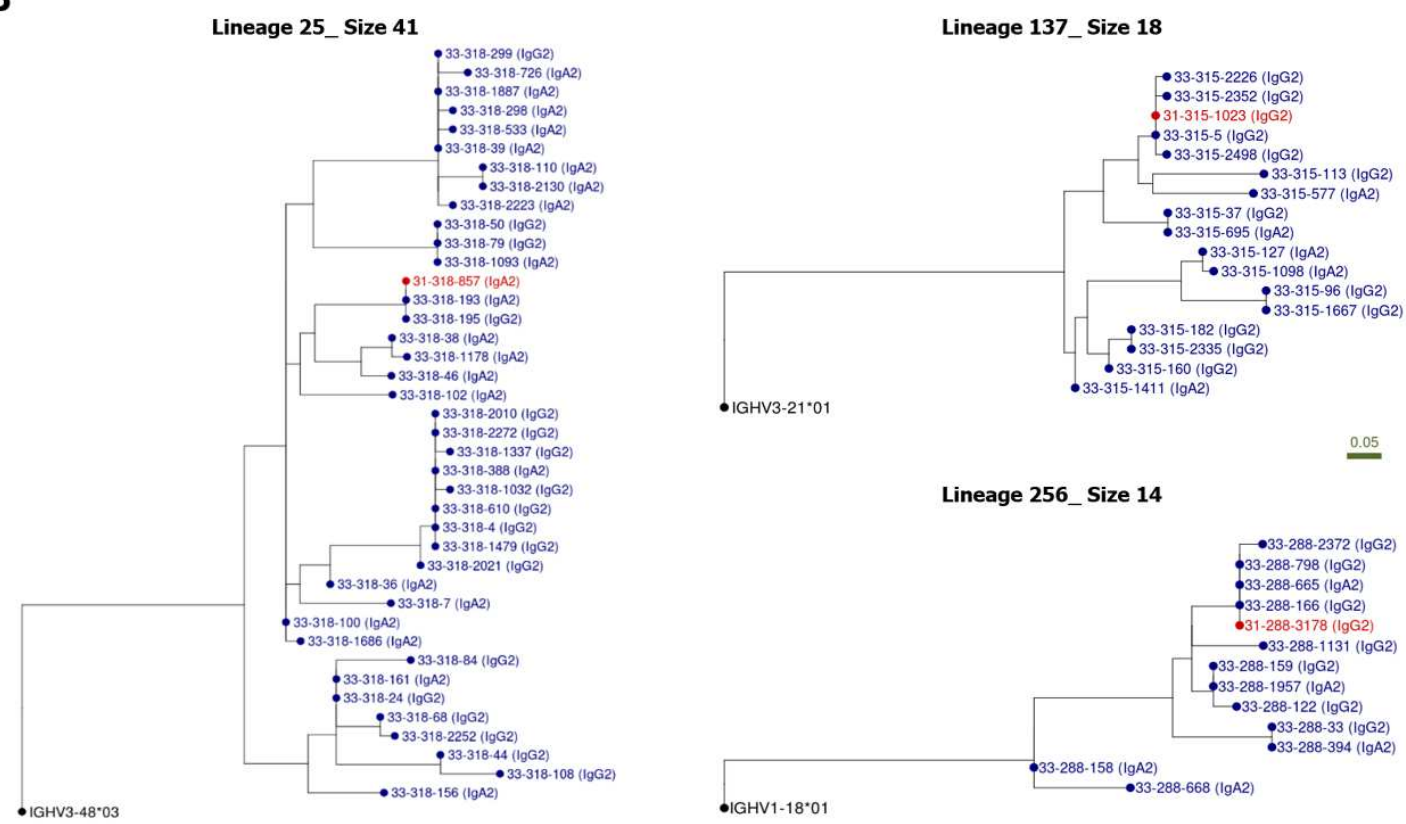

Supplementary Fig. 7. Phylogenetic analysis for the representative BCR lineages composed of the highly similar overlapped BCR sequences Phylogenetic analysis was conducted for the representative BCR lineages composed of the highly similar overlapped BCR sequences. The BCR lineages were categorized into two groups, (A) IgM-major lineages and Class-switched lineages. The ID of BCR lineages and size of them were represented on the top of the phylogenetic trees. Isotypes of the sequences were denoted in the parenthesis of the sequence names. The green scale bars on the right bottom of the trees represent the intersequence distance calculated by the IgPhyML phylogenetic tree construction tool. 
- $A D \& A D=$ Normal \& $X$
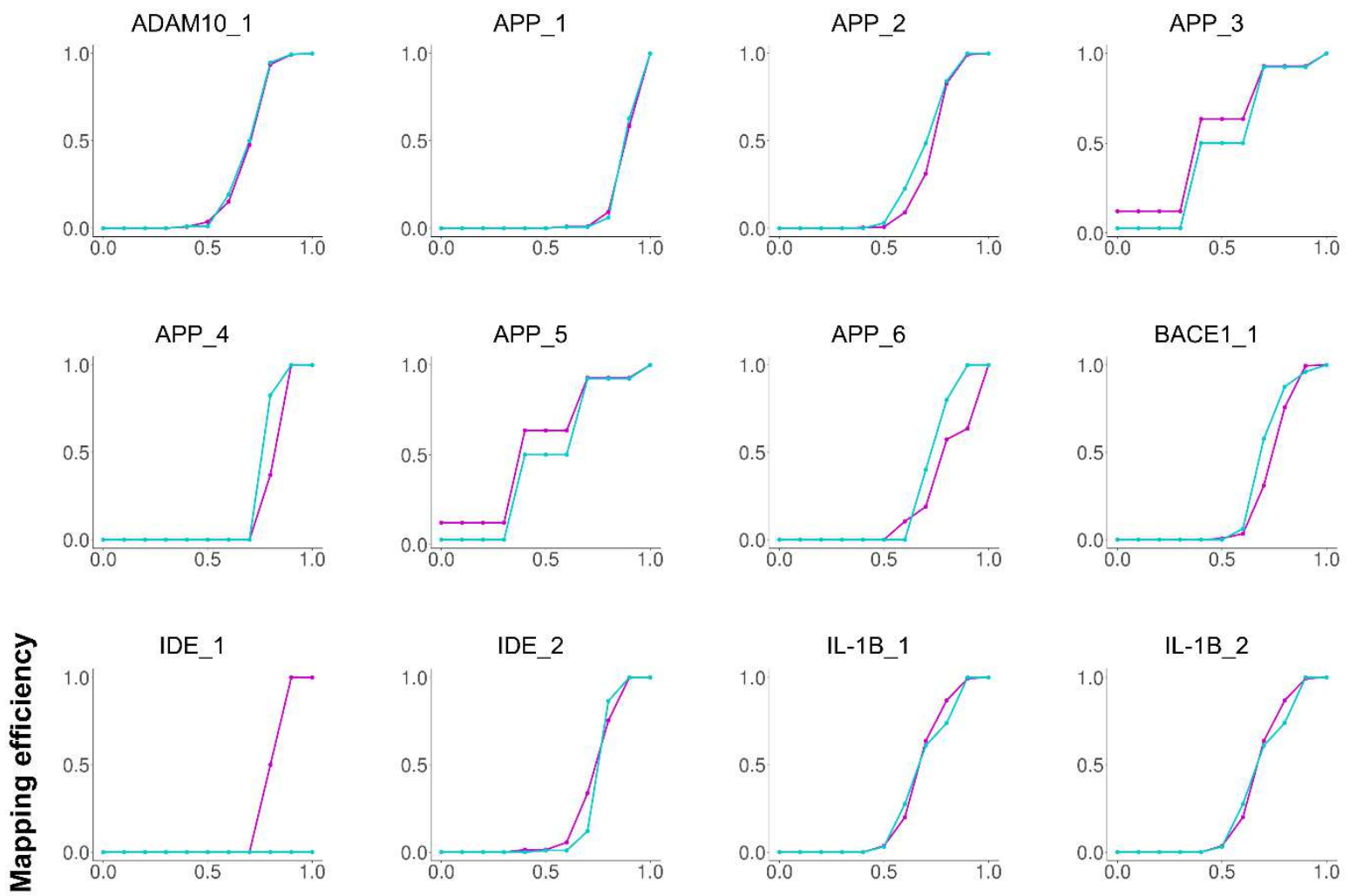

IL-6_1

IL-12_1

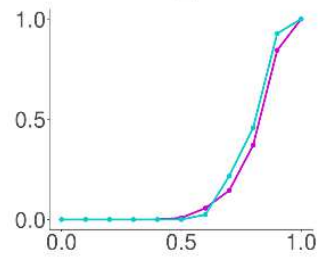

IL-15_1

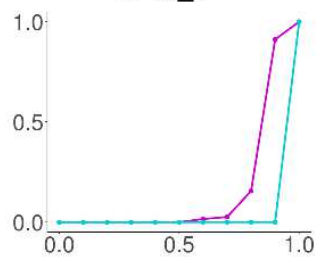

IL-13_1

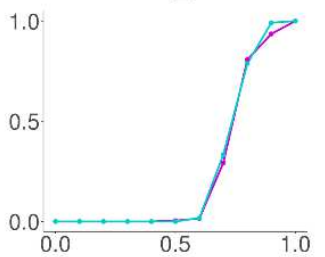

IL-17A_1

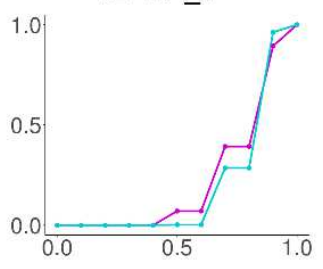

IL-13_2

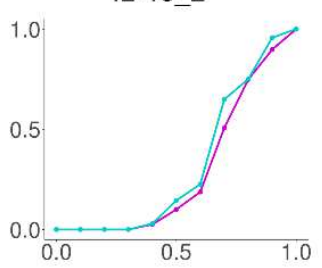

IL-17A_2

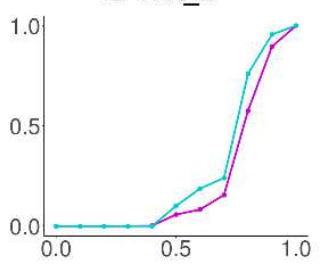

CDR3 AA mismatch threshold

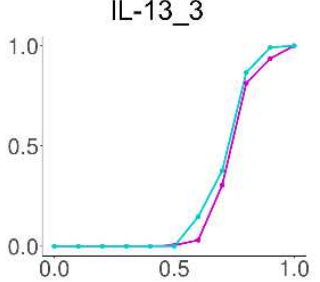

Supplementary Fig. 8-1. In silico mapping results of 39 BCR sequences (whole sequences overlapped) binding to AD-related proteins (related to Fig. 4). 
- $A D \& A D=$ Normal \& $X$

IL-17A_3

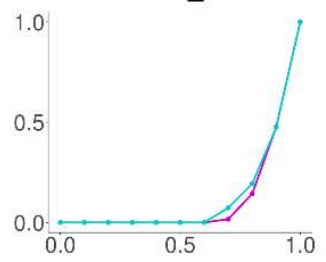

LPL_1
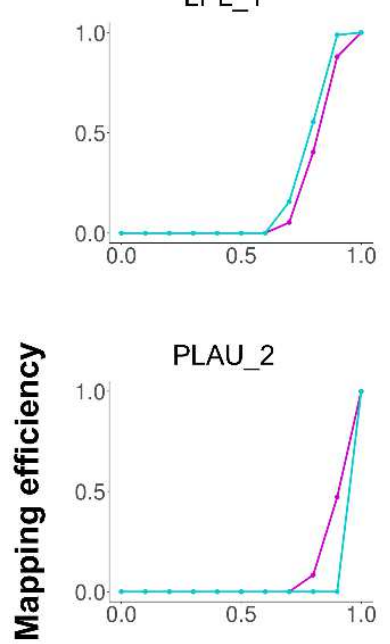

PRNP_3

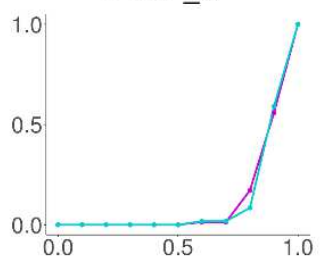

PRNP 3

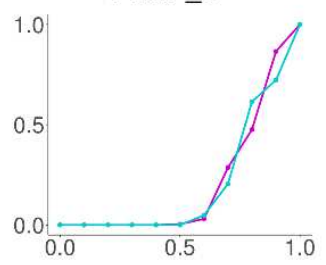

IL-22_1

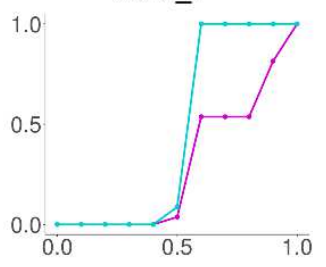

MAPT_1

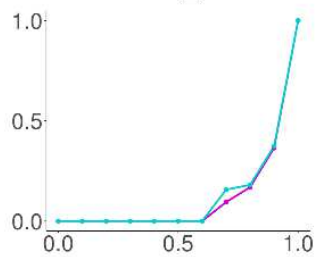

PLAU_3

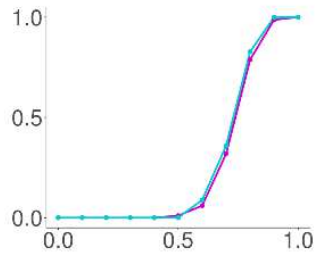

PRNP_4

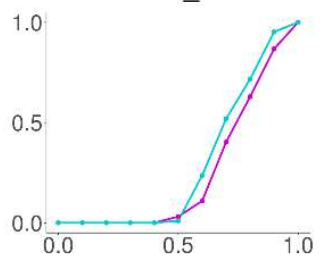

PRNP 4

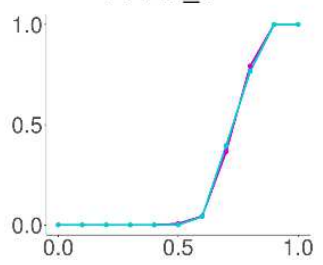

IL-34_1

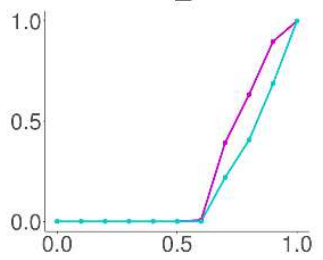

MAPT_2

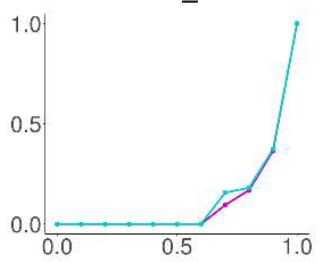

PRNP_1

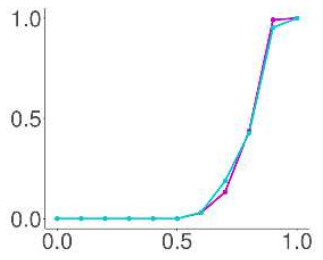

PRNP_5

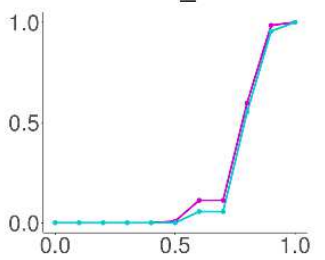

PRNP 5

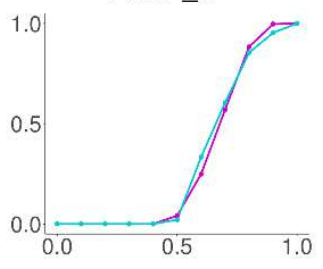

IL-34_2

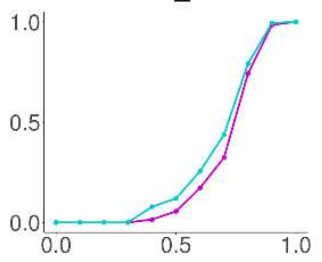

PLAU_1

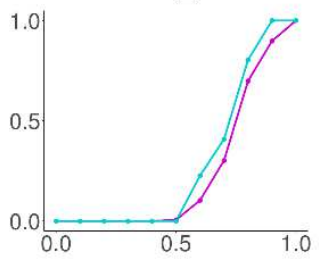

PRNP_2

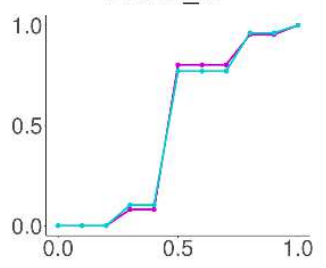

SNCA_1

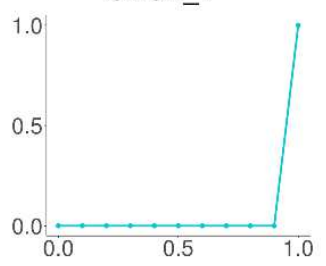

CDR3 AA mismatch threshold

119 Supplementary Fig. 8-2. In silico mapping results of 39 BCR sequences (whole sequences overlapped) binding to AD-related proteins (related to Fig. 4). 
- $A D \& A D=$ Normal \& $X$

ADAM10_1

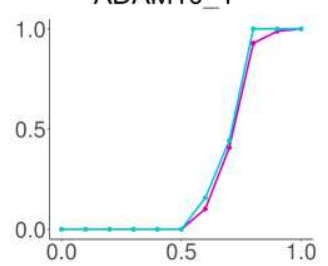

APP_4
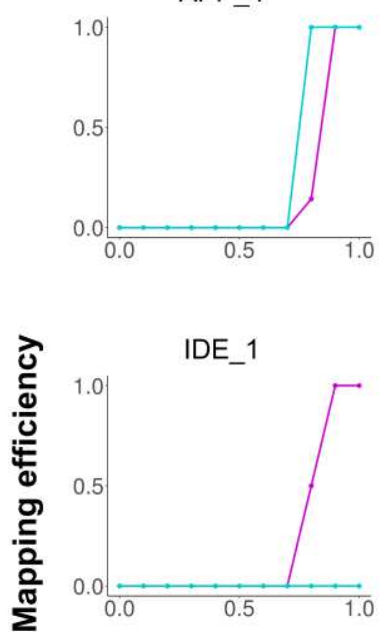

IL-6_1

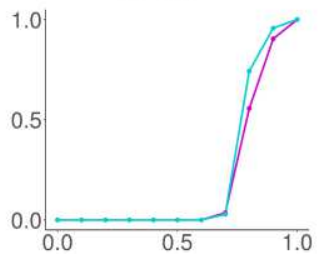

IL-13_3

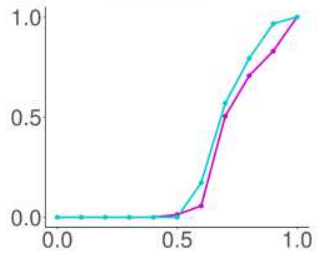

APP_1

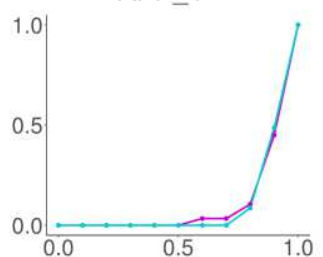

APP_5

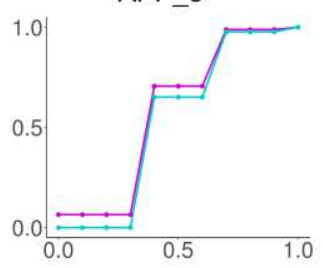

IDE 2

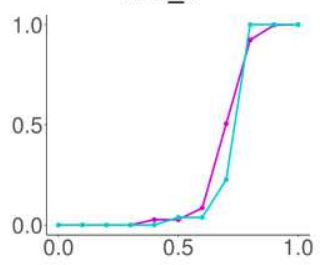

IL-12_1

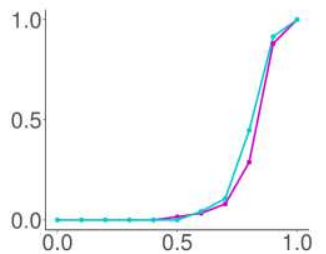

IL-15_1

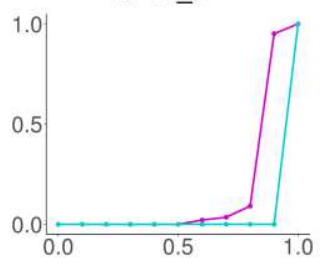

APP_2

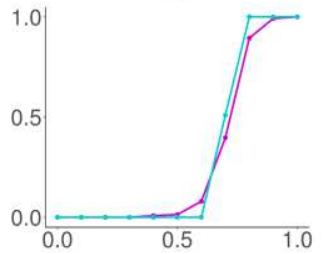

APP_6

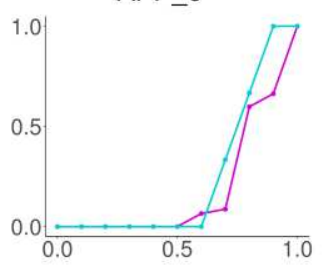

IL-1B_1

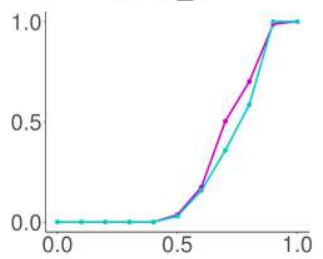

IL-13_1

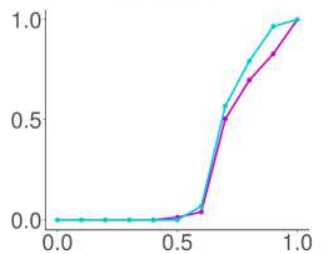

IL-17A_1

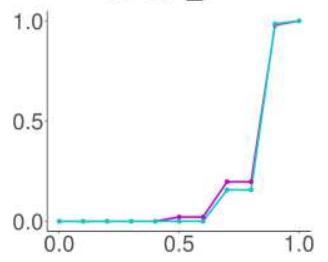

APP_3

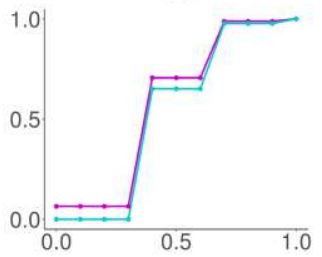

BACE1_1

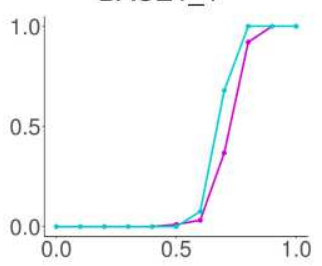

IL-1B_2

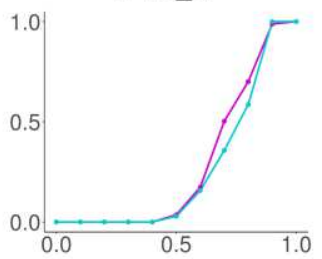

IL-13_2

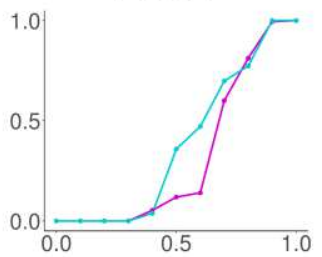

IL-17A_2

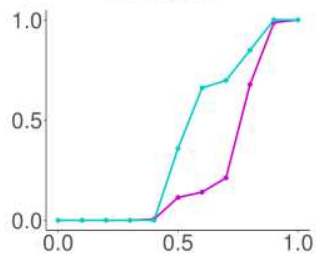

CDR3 AA mismatch threshold

123 Supplementary Fig. 9-1. In silico mapping results of 39 BCR sequences (highly similar shared) binding to AD-related proteins (related to Fig. 4). 
- $A D \& A D=$ Normal \& $X$

IL-17A_3

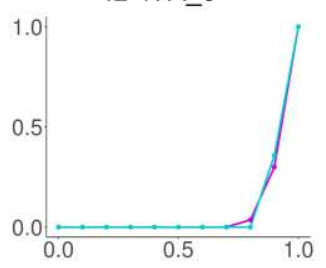

LPL_1
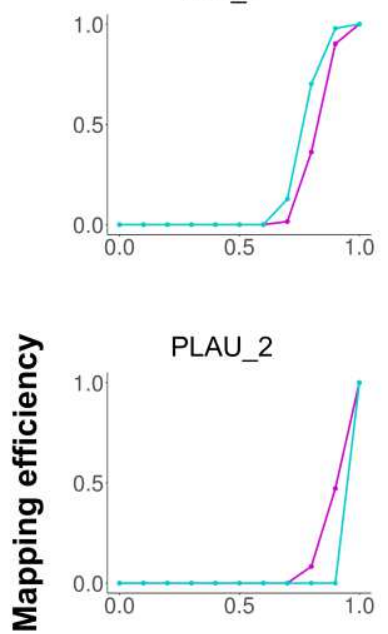

PRNP_3

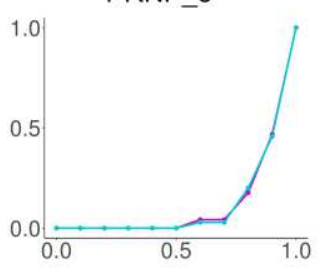

PRNP_3

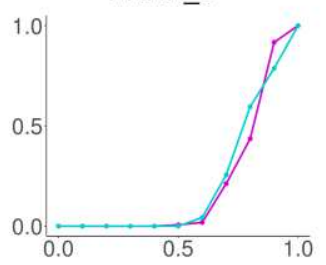

IL-22_1

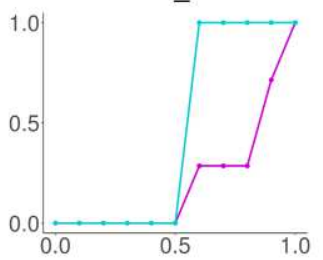

MAPT 1

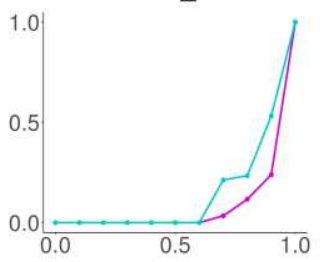

PLAU_3

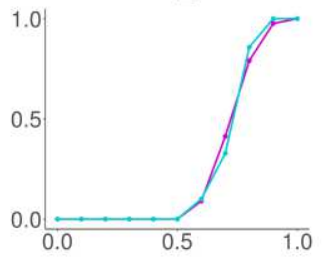

PRNP_4

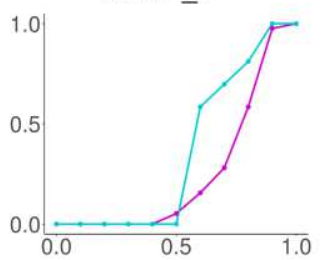

PRNP_4

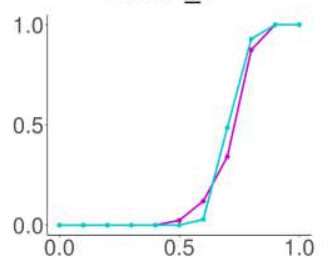

IL-34_1

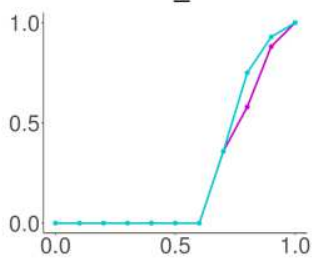

MAPT 2

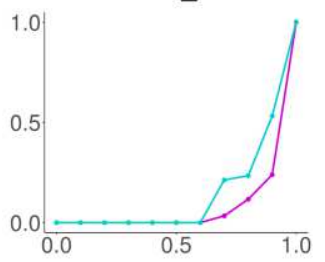

PRNP_1

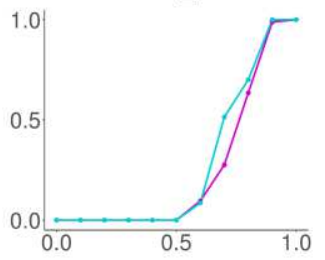

PRNP_5

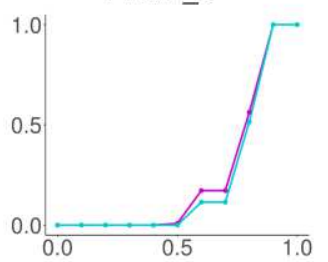

PRNP_5

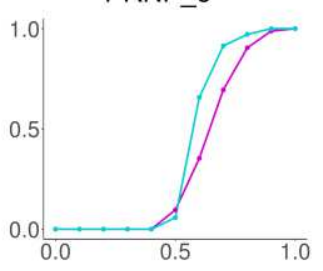

IL-34_2

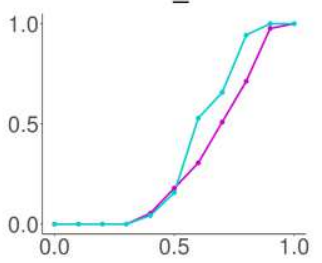

PLAU_1

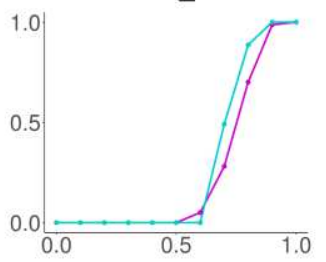

PRNP_2

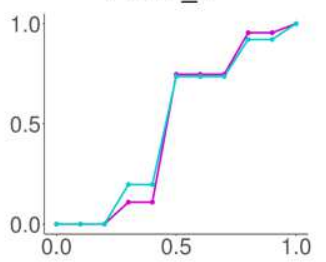

SNCA_1

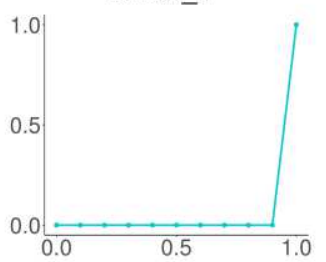

CDR3 AA mismatch threshold

127 Supplementary Fig. 9-2. In silico mapping results of 39 BCR sequences (highly similar shared) binding to AD-related proteins (related to Fig. 4). 


\begin{tabular}{|c|c|c|c|}
\hline Reagent & Supplier & Catalog number & etc. \\
\hline BD K2 EDTA tubes & BD Bioscience & 368861 & \\
\hline $\begin{array}{l}\text { Mr. Frosty Freezing } \\
\text { Container }\end{array}$ & Thermo Scientific & 5100-0001 & \\
\hline $\begin{array}{l}\text { FITC anti-human CD3 } \\
\text { antibody }\end{array}$ & BioLegend & 317306 & $\begin{array}{l}\text { Dilution, 1:100; Clone, } \\
\text { OKT3 }\end{array}$ \\
\hline $\begin{array}{l}\text { PE anti-human CD19 } \\
\text { antibody }\end{array}$ & BioLegend & 302208 & $\begin{array}{l}\text { Dilution, 1:100; Clone, } \\
\text { HIB19 }\end{array}$ \\
\hline $\begin{array}{l}\text { APC/Cy7 anti-human } \\
\text { CD16 antibody }\end{array}$ & BioLegend & 302018 & $\begin{array}{l}\text { Dilution, 1:100; Clone, } \\
\text { 3G8 }\end{array}$ \\
\hline $\begin{array}{l}\text { APC anti-human CD33 } \\
\text { antibody }\end{array}$ & BioLegend & 303408 & $\begin{array}{l}\text { Dilution, 1:100; Clone, } \\
\text { WM53 }\end{array}$ \\
\hline $\begin{array}{l}\text { Human TruStain FcX } \\
\text { (Fc Receptor blocking } \\
\text { solution) }\end{array}$ & BioLegend & 422302 & $\begin{array}{l}5 \mu \mathrm{m} / \text { million cells in } 100 \\
\mu \mathrm{l} \text { staining volume }\end{array}$ \\
\hline RPMI1640 media & Gibco & $11875-093$ & \\
\hline FBS & Gibco & 26140079 & \\
\hline HBSS media & WELGENE & LB003-02 & \\
\hline DAPI reagent & Sigma Aldrich & D9542 & $400 \mathrm{ng} / \mathrm{ml}$ \\
\hline FACS tube & BD Bioscience & 352052 & \\
\hline FACS tube strainer & BD Bioscience & 352235 & $335 \mu \mathrm{m}$ nylon mesh \\
\hline
\end{tabular}


134 Supplementary Table 2. Statistics for BCR repertoire NGS data

\begin{tabular}{|c|c|c|c|}
\hline Identifier & UMI-processed read & $\begin{array}{c}\text { Unique consensus } \\
\text { sequence \# }\end{array}$ & B cell lineage \# \\
\hline N01 & 292,035 & 37,048 & 30,112 \\
\hline N02 & 184,553 & 40,905 & 33,765 \\
\hline N03 & 266,446 & 50,090 & 43,203 \\
\hline AD01 & 160,743 & 51,240 & 46,161 \\
\hline AD02 & 465,866 & 265,763 & 255,041 \\
\hline AD03 & 319,869 & 87,427 & 78,126 \\
\hline AD04 & 548,559 & 129,448 & 106,345 \\
\hline AD05 & 319,839 & 112,895 & 104,610 \\
\hline AD06 & 345,679 & 103,515 & 94,390 \\
\hline AD07 & 683,273 & 309,252 & 279,808 \\
\hline AD08 & 738,650 & 246,287 & 205,783 \\
\hline AD09 & 382,745 & 83,242 & 61,505 \\
\hline AD10 & 456,861 & 135,060 & 96,408 \\
\hline
\end{tabular}


137 Supplementary Table 3. Class-switched and highly similar overlapped BCR lineages

\begin{tabular}{|c|c|c|c|c|c|c|c|}
\hline CDR3 AA & $V$ gene & $\mathbf{J}$ gene & Sequence \# & Divergence & Readcount & Isotype & Sample \\
\hline QGVFGGDHEGPGDFDF & IGHV3-23 & IGHJ4 & 1 & $0.17+-0.0$ & $1.0+-0.0$ & G3 & AD10 \\
\hline QGVFGGDHEGPGDFDF & IGHV3-23 & IGHJ4 & 90 & $0.17+-0.01$ & $33.99+-303.02$ & G3 & AD07 \\
\hline AERRGTVRGVINHYYYHNFGMEV & IGHV3-13 & IGHJ6 & 1 & $0.16+-0.0$ & $1.0+-0.0$ & A1 & AD10 \\
\hline AERRGTVRGVINHSYYHNFGMDV & IGHV3-13 & IGHJ6 & 6 & $0.16+-0.0$ & $17.0+-11.65$ & A1 & AD07 \\
\hline QRRKIPVALPGSLATFDM & IGHV1-46 & IGHJ3 & 1 & $0.13+-0.0$ & $1.0+-0.0$ & A2 & AD10 \\
\hline QRRKIPVALPGSLATFDM & IGHV1-46 & IGHJ3 & 5 & $0.13+-0.0$ & $29.8+-57.6$ & A2 & AD07 \\
\hline HKMGTRSFDY & IGHV4-59 & IGHJ4 & 1 & $0.09+-0.0$ & $1.0+-0.0$ & A2 & AD10 \\
\hline HKMGTRSFDY & IGHV4-59 & IGHJ4 & 3 & $0.09+-0.0$ & $41.33+-57.04$ & $\mathrm{~A} 2$ & AD07 \\
\hline GGSSWYIGRD & IGHV1-69 & IGHJ4 & 1 & $0.05+-0.0$ & $1.0+-0.0$ & G1 & $\mathrm{AD} 10$ \\
\hline GGSSWYIGRD & IGHV1-69 & IGHJ4 & 2 & $0.05+-0.0$ & $16.0+-15.0$ & G1 & AD07 \\
\hline LGMAARIIDY & IGHV4-31 & IGHJ4 & 1 & $0.15+-0.0$ & $1.0+-0.0$ & G1 & AD10 \\
\hline LGMAARIIDY & IGHV4-31 & IGHJ4 & 2 & $0.15+-0.0$ & $86.0+-85.0$ & G1 & AD07 \\
\hline RGNSAGWYYFDE & IGHV3-23 & IGHJ4 & 1 & $0.12+-0.0$ & $1.0+-0.0$ & A1 & $\mathrm{AD} 10$ \\
\hline RGNSAGWYYFDE & IGHV3-23 & IGHJ4 & 1 & $0.12+-0.0$ & $9.0+-0.0$ & A1 & AD07 \\
\hline DGCSSTWERNGFHP & IGHV4-39 & IGHJ5 & 1 & $0.11+-0.0$ & $1.0+-0.0$ & A1 & AD10 \\
\hline DGCSSTWERNGFHP & IGHV4-39 & IGHJ5 & 1 & $0.1+-0.0$ & $16.0+-0.0$ & A1 & AD07 \\
\hline EGYGYVLGIDY & IGHV4-4 & IGHJ4 & 1 & $0.07+-0.0$ & $1.0+-0.0$ & A1 & $\mathrm{AD} 10$ \\
\hline EGYGYVLGIDY & IGHV4-4 & IGHJ4 & 1 & $0.07+-0.0$ & $13.0+-0.0$ & A1 & AD07 \\
\hline DLDDSLEGGAMGV & IGHV3-7 & IGHJ6 & 30 & $0.04+-0.0$ & $74.33+-394.36$ & G1 & AD06 \\
\hline DLDDSLEGGAMGV & IGHV3-7 & IGHJ6 & 1 & $0.04+-0.0$ & $3.0+-0.0$ & G1 & AD04 \\
\hline GGWGDLSPDY & IGHV1-18 & IGHJ4 & 1 & $0.09+-0.0$ & $1.0+-0.0$ & G2 & AD04 \\
\hline GGWGDLSPDY & IGHV1-18 & IGHJ4 & 14 & $0.08+-0.01$ & $9.79+-8.11$ & $\mathrm{G} 2 \mid \mathrm{A} 2$ & AD06 \\
\hline HWGSEHYDYSFDS & IGHV3-23 & IGHJ4 & 9 & $0.11+-0.02$ & $67.56+-187.89$ & G2 & AD06 \\
\hline HWGSEHYDYSFDS & IGHV3-23 & IGHJ4 & 1 & $0.11+-0.0$ & $1.0+-0.0$ & $\mathrm{G} 2$ & AD04 \\
\hline DFRWAFDL & IGHV6-1 & IGHJ3 & 1 & $0.14+-0.0$ & $1.0+-0.0$ & $\mathrm{~A} 2$ & AD04 \\
\hline DFRWAFDL & IGHV6-1 & IGHJ3 & 11 & $0.14+-0.01$ & $28.91+-33.3$ & $\mathrm{G} 2 \mid \mathrm{A} 2$ & AD06 \\
\hline ERQIWGASDY & IGHV3-23 & IGHJ4 & 2 & $0.13+-0.01$ & $1.0+-0.0$ & G1 & AD04 \\
\hline ERQIWGASDY & IGHV3-23 & IGHJ4 & 7 & $0.13+-0.01$ & $19.29+-30.83$ & G1 & AD06 \\
\hline DAFGRADS & IGHV3-74 & IGHJ4 & 2 & $0.13+-0.0$ & $1.0+-0.0$ & $\mathrm{G} 2$ & AD04 \\
\hline DAFGRADS & IGHV3-74 & IGHJ4 & 8 & $0.14+-0.0$ & $4.88+-10.25$ & $\mathrm{G} 2 \mid \mathrm{A} 2$ & AD06 \\
\hline GWDEGYFYYYMDV & IGHV3-23 & IGHJ6 & 1 & $0.04+-0.0$ & $1.0+-0.0$ & G2 & AD04 \\
\hline GWDEGYFYYYMDV & IGHV3-23 & IGHJ6 & 5 & $0.05+-0.0$ & $34.8+-67.6$ & $\mathrm{G} 2$ & AD06 \\
\hline GSHISGTTKVLFDY & IGHV3-74 & IGHJ4 & 1 & $0.02+-0.0$ & $1.0+-0.0$ & $\mathrm{G} 2$ & AD04 \\
\hline GSHISGTTKVLFDY & IGHV3-74 & IGHJ4 & 4 & $0.02+-0.0$ & $42.5+-71.88$ & G2 & AD06 \\
\hline HGLYGGNGYHWGH & IGHV3-23 & IGHJ4 & 1 & $0.1+-0.0$ & $1.0+-0.0$ & $\mathrm{G} 2$ & AD04 \\
\hline HGLYGGNGYHWGH & IGHV3-23 & IGHJ4 & 4 & $0.1+-0.0$ & $47.75+-80.97$ & G2 & AD06 \\
\hline GSGWVADI & IGHV3-7 & IGHJ3 & 1 & $0.07+-0.0$ & $1.0+-0.0$ & $\mathrm{G} 2$ & AD04 \\
\hline GSGWVADI & IGHV3-7 & IGHJ3 & 4 & $0.08+-0.0$ & $27.5+-45.9$ & G2 & AD06 \\
\hline NTGRGTGLERPWDWFDP & IGHV3-7 & IGHJ5 & 1 & $0.09+-0.0$ & $1.0+-0.0$ & $\mathrm{G} 2$ & AD04 \\
\hline NTGRGTGLERPWDWFDP & IGHV3-7 & IGHJ5 & 4 & $0.09+-0.0$ & $8.0+-9.46$ & $\mathrm{G} 2$ & AD06 \\
\hline ALEYARGSNFYYGLDV & IGHV4-34 & IGHJ6 & 1 & $0.12+-0.0$ & $1.0+-0.0$ & A1 & AD04 \\
\hline ALEYARGSNFYYGLDV & IGHV4-34 & IGHJ6 & 3 & $0.12+-0.0$ & $6.33+-7.54$ & A1 & AD06 \\
\hline GRVLVRGSGYFDS & IGHV4-34 & IGHJ4 & 1 & $0.13+-0.0$ & $1.0+-0.0$ & G1 & AD04 \\
\hline GRVLVRGSGYFDS & IGHV4-34 & IGHJ4 & 3 & $0.13+-0.0$ & $47.33+-65.53$ & G1 & AD06 \\
\hline DDNYGTFTYYYGMDV & IGHV3-30 & IGHJ6 & 1 & $0.13+-0.0$ & $1.0+-0.0$ & $\mathrm{~A} 2$ & AD04 \\
\hline DDNYGTFTYYYGMDV & IGHV3-30 & IGHJ6 & 3 & $0.13+-0.0$ & $10.0+-12.03$ & A2 & AD06 \\
\hline RNSSGPWAFDI & IGHV3-23 & IGHJ3 & 1 & $0.09+-0.0$ & $1.0+-0.0$ & A2 & AD04 \\
\hline RNSSGPWAFDI & IGHV3-23 & IGHJ3 & 2 & $0.1+-0.0$ & $25.0+-24.0$ & $\mathrm{~A} 2$ & AD06 \\
\hline GGLIALDY & IGHV3-49 & IGHJ4 & 1 & $0.06+-0.0$ & $1.0+-0.0$ & $\mathrm{G} 2$ & AD04 \\
\hline GGLIALDY & IGHV3-49 & IGHJ4 & 3 & $0.06+-0.0$ & $26.33+-35.83$ & G2 & AD06 \\
\hline GINGYFDTSGSFDY & IGHV1-69 & IGHJ4 & 1 & $0.09+-0.0$ & $1.0+-0.0$ & G1 & AD04 \\
\hline GINGYFDTSGSFDY & IGHV1-69 & IGHJ4 & 3 & $0.09+-0.0$ & $18.33+-24.51$ & G1 & AD06 \\
\hline GLGYSSSS & IGHV3-74 & IGHJ5 & 1 & $0.12+-0.0$ & $1.0+-0.0$ & G2 & AD04 \\
\hline GLGYSSSS & IGHV3-74 & IGHJ5 & 4 & $0.11+-0.01$ & $33.75+-56.72$ & G2|A2 & AD06 \\
\hline GGDPDS & IGHV4-4 & IGHJ5 & 1 & $0.05+-0.0$ & $1.0+-0.0$ & $\mathrm{~A} 1$ & AD04 \\
\hline GGDPDS & IGHV4-4 & IGHJ5 & 3 & $0.05+-0.0$ & $8.67+-10.14$ & $\mathrm{~A} 1 \mid \mathrm{A} 2$ & AD06 \\
\hline GEDSGSYIIDY & IGHV1-3 & IGHJ4 & 1 & $0.1+-0.0$ & $1.0+-0.0$ & $\mathrm{G} 2$ & AD04 \\
\hline GEDSGSYIIDY & IGHV1-3 & IGHJ4 & 2 & $0.1+-0.0$ & $14.5+-13.5$ & $\mathrm{G} 2$ & AD06 \\
\hline GVKVRGVIKDY & IGHV4-4 & IGHJ4 & 1 & $0.03+-0.0$ & $1.0+-0.0$ & $\mathrm{G} 2$ & AD04 \\
\hline GVKVRGVIKDY & IGHV4-4 & IGHJ4 & 2 & $0.03+-0.0$ & $92.0+-91.0$ & $\mathrm{G} 2$ & AD06 \\
\hline EYNDDVWGSHLNHYYMDV & IGHV1-8 & IGHJ6 & 1 & $0.14+-0.0$ & $1.0+-0.0$ & G1 & AD04 \\
\hline EYNDDVWGSHLNHYYMDV & IGHV1-8 & IGHJ6 & 2 & $0.14+-0.0$ & $15.0+-14.0$ & G1 & AD06 \\
\hline GYYHDSSGYRGL & IGHV4-4 & IGHJ4 & 1 & $0.06+-0.0$ & $1.0+-0.0$ & G1 & AD04 \\
\hline GYYHDSSGYRGL & IGHV4-4 & IGHJ4 & 2 & $0.06+-0.0$ & $31.5+-30.5$ & G1 & AD06 \\
\hline EKVNSGTFDY & IGHV1-3 & IGHJ4 & 1 & $0.11+-0.0$ & $1.0+-0.0$ & A1 & AD04 \\
\hline EKVNSGTFDY & IGHV1-3 & IGHJ4 & 1 & $0.11+-0.0$ & $4.0+-0.0$ & A1 & AD06 \\
\hline DGGMLHQNYYRNV & IGHV3-23 & IGHJ6 & 1 & $0.1+-0.0$ & $1.0+-0.0$ & $\mathrm{G} 2$ & AD04 \\
\hline DGGMLHQNYYRNV & IGHV3-23 & IGHJ6 & 2 & $0.11+-0.0$ & $20.0+-19.0$ & G2 & AD06 \\
\hline VLWNAGRLFDY & IGHV1-18 & IGHJ4 & 1 & $0.06+-0.0$ & $1.0+-0.0$ & A2 & AD04 \\
\hline VLWNAGRLFDY & IGHV1-18 & IGHJ4 & 2 & $0.06+-0.0$ & $29.0+-28.0$ & A2 & AD06 \\
\hline GGGYQLPPRGMDV & IGHV3-30 & IGHJ6 & 1 & $0.0+-0.0$ & $1.0+-0.0$ & G1 & AD04 \\
\hline GGGYQLPPRGMDV & IGHV3-30 & IGHJ6 & 2 & $0.0+-0.0$ & $3.0+-0.0$ & G1 & AD06 \\
\hline AGGFYGDILY & IGHV1-46 & IGHJ4 & 2 & $0.19+-0.0$ & $2.5+-1.5$ & A2 & AD06 \\
\hline AGGFYGDILY & IGHV1-46 & IGHJ4 & 1 & $0.19+-0.0$ & $1.0+-0.0$ & $\mathrm{~A} 2$ & AD04 \\
\hline DPPQSGYSLDC & IGHV3-33 & IGHJ4 & 1 & $0.05+-0.0$ & $1.0+-0.0$ & $\mathrm{~A} 2$ & AD04 \\
\hline DPPQSGYSLDC & IGHV3-33 & IGHJ4 & 2 & $0.05+-0.0$ & $26.5+-1.5$ & A2 & AD06 \\
\hline
\end{tabular}




\begin{tabular}{|c|c|c|}
\hline EMYSSGSGTFTWYFAMDV & IGHV4-4 & IGHJ6 \\
\hline EMYSSGSGTFTWYFAMDV & IGHV4-4 & IGHJ6 \\
\hline EYYYDSRGFKPTGFDS & IGHV3-30 & IGHJ4 \\
\hline EYYYDSRGFKPTGFDS & IGHV3-30 & IGHJ4 \\
\hline ESLHGSGSYYIRAFDI & IGHV3-21 & IGHJ3 \\
\hline ESLHGSGSYYIRAFDI & IGHV3-21 & IGHJ3 \\
\hline KGQGGLAHFDY & IGHV2-5 & IGHJ4 \\
\hline KGQGGLAHFDY & IGHV2-5 & IGHJ4 \\
\hline NPQARAASTINWYFEL & IGHV3-30 & IGHJ2 \\
\hline NPQARAASTINWYFEL & IGHV3-30 & IGHJ2 \\
\hline HTDHVVVLGFSTVAAFDM & IGHV3-49 & IGHJ3 \\
\hline HTDHVVVLGFSTVAAFDM & IGHV3-49 & IGHJ3 \\
\hline GPIVVGPLDP & IGHV3-13 & IGHJ5 \\
\hline GPIVVGPLDP & IGHV3-13 & IGHJ5 \\
\hline SQLLIGGSGWELDS & IGHV3-30-3 & IGHJ5 \\
\hline SQLLIGGSGWELDS & IGHV3-30-3 & IGHJ5 \\
\hline AKYTYSPGIHFDY & IGHV4-59 & IGHJ4 \\
\hline AKYTYSPGIHFDY & IGHV4-59 & IGHJ4 \\
\hline MTVGNWFDP & IGHV4-39 & IGHJ5 \\
\hline MTVGNWFDP & IGHV4-39 & IGHJ5 \\
\hline DQAARVPWYFDH & IGHV1-18 & IGHJ4 \\
\hline DQAARVPWYFDH & IGHV1-18 & IGHJ4 \\
\hline APKGAGGSY & IGHV3-7 & IGHJ4 \\
\hline APKGAGGSY & IGHV3-7 & IGHJ4 \\
\hline ASARTYTAYGVALSHMDV & IGHV3-23 & IGHJ6 \\
\hline ASARTYTAYGVALSHMDV & IGHV3-23 & IGHJ6 \\
\hline SPYYYASGGSTFFEY & IGHV3-23 & IGHJ4 \\
\hline SPYYYASGGSTFFEY & IGHV3-23 & IGHJ4 \\
\hline DLPVGFGSSAY & IGHV1-46 & IGHJ4 \\
\hline DLPVGFGSSAY & IGHV1-46 & IGHJ4 \\
\hline FEGMESSAYYFDS & IGHV3-23 & IGHJ4 \\
\hline FEGMESSAYYFDS & IGHV3-23 & IGHJ4 \\
\hline QRAAGYYYYYYMDV & IGHV3-23 & IGHJ6 \\
\hline QRAAGYYYYYYMDV & IGHV3-23 & IGHJ6 \\
\hline DRAGRKAPRSLYFDY & IGHV3-11 & IGHJ4 \\
\hline DRAGRKAPRSLYFDY & IGHV3-11 & IGHJ4 \\
\hline DRMFFDS & IGHV3-23 & IGHJ4 \\
\hline DRMFFDS & IGHV3-23 & IGHJ4 \\
\hline HRGPPQEWPPKYWFDP & IGHV4-39 & IGHJ5 \\
\hline HRGPPQEWPPKYWFDP & IGHV4-39 & IGHJ5 \\
\hline HLRRYTYGYYFDF & IGHV4-39 & IGHJ4 \\
\hline HLRRYTYGYYFDF & IGHV4-39 & IGHJ4 \\
\hline DGAAYVHYHYGMDV & IGHV3-49 & IGHJ6 \\
\hline DGAAYVHYHYGMDV & IGHV3-49 & IGHJ6 \\
\hline SLVAVQLPHYYYAMDV & IGHV3-53 & IGHJ6 \\
\hline SLVAVQLPHYYYAMDV & IGHV3-53 & IGHJ6 \\
\hline GQDQYKTGNY & IGHV4-34 & IGHJ4 \\
\hline GQDQYKTGNY & IGHV4-34 & IGHJ4 \\
\hline SWTATSGYCNWFDS & IGHV2-5 & IGHJ5 \\
\hline SWTATSGYCNWFDS & IGHV2-5 & IGHJ5 \\
\hline GLFCIDGDCFSSWFDS & IGHV3-48 & IGHJ5 \\
\hline GLFCIDGDCFSSWFDS & IGHV3-48 & IGHJ5 \\
\hline ALFGPGPTSFDY & IGHV1-24 & IGHJ4 \\
\hline ALFGPGPTSFDY & IGHV1-24 & IGHJ4 \\
\hline EYFGVADH & IGHV3-49 & IGHJ4 \\
\hline EYFGVADH & IGHV3-49 & IGHJ4 \\
\hline VPRAFSAPYYYYGMDV & IGHV1-46 & IGHJ6 \\
\hline VPRAFSAPYYYYGMDV & IGHV1-46 & IGHJ6 \\
\hline WNSGSCSSF & IGHV3-49 & IGHJ4 \\
\hline WNSGSCSSF & IGHV3-49 & IGHJ4 \\
\hline DWEYHLQD & IGHV4-4 & IGHJ4 \\
\hline DWEYHLQD & IGHV4-4 & IGHJ4 \\
\hline DSTRAYSHDYLRGWFDP & IGHV3-23 & IGHJ5 \\
\hline DSTRAYSHDYLRGWFDP & IGHV3-23 & IGHJ5 \\
\hline EHCGGGGCYSLDY & IGHV3-74 & IGHJ4 \\
\hline EHCGGGGCYSLDY & IGHV3-74 & IGHJ4 \\
\hline YSSEHFYGLDV & IGHV1-69 & IGHJ6 \\
\hline YSSEHFYGLDV & IGHV1-69 & IGHJ6 \\
\hline GGHYFGADV & IGHV1-3 & IGHJ6 \\
\hline GGHYFGADV & IGHV1-3 & IGHJ6 \\
\hline HTGSSLRIDY & IGHV1-69 & IGHJ4 \\
\hline HTGSSLRIDY & IGHV1-69 & IGHJ4 \\
\hline GHLYDFWSGPLFDY & IGHV1-24 & IGHJ4 \\
\hline GHLYDFWSGPLFDY & IGHV1-24 & IGHJ4 \\
\hline LRYCSMTRCYRVDY & IGHV1-24 & IGHJ4 \\
\hline LRYCSMTRCYRVDY & IGHV1-24 & IGHJ4 \\
\hline DLSGEASLDGR & IGHV3-72 & IGHJ3 \\
\hline DLSGEASLDGR & IGHV3-72 & IGHJ3 \\
\hline LDYSHGSTYSVPPV & IGHV3-64 & IGHJ6 \\
\hline LDYSHGSTYSVPPV & IGHV3-64 & IGHJ6 \\
\hline QVLGEADYSDSVGSYYVDYF & IGHV4-39 & IGHJ4 \\
\hline
\end{tabular}

\begin{tabular}{|c|c|c|c|}
\hline $0.09+-0.0$ & $1.0+-0.0$ & G2 & AD04 \\
\hline $0.09+-0.0$ & $38.0+-37.0$ & G2 & AD06 \\
\hline $0.07+-0.0$ & $1.0+-0.0$ & G2 & AD04 \\
\hline $0.07+-0.0$ & $59.0+-58.0$ & G2 & AD06 \\
\hline $0.03+-0.0$ & $1.0+-0.0$ & G1 & $\mathrm{AD} 04$ \\
\hline $0.04+-0.0$ & $1.5+-0.5$ & G1 & AD06 \\
\hline $0.04+-0.0$ & $1.0+-0.0$ & G2 & AD04 \\
\hline $0.04+-0.0$ & $29.5+-28.5$ & G2 & AD06 \\
\hline $0.07+-0.0$ & $1.0+-0.0$ & G2 & AD04 \\
\hline $0.07+-0.0$ & $44.5+-43.5$ & G2 & AD06 \\
\hline $0.16+-0.0$ & $1.0+-0.0$ & G2 & AD04 \\
\hline $0.16+-0.0$ & $3.0+-1.0$ & G2 & AD06 \\
\hline $0.09+-0.0$ & $1.0+-0.0$ & G2 & AD04 \\
\hline $0.1+-0.0$ & $54.5+-53.5$ & G2 & AD06 \\
\hline $0.16+-0.0$ & $1.0+-0.0$ & A2 & AD04 \\
\hline $0.16+-0.0$ & $6.5+-1.5$ & A2 & AD06 \\
\hline $0.07+-0.0$ & $1.0+-0.0$ & G1 & AD04 \\
\hline $0.07+-0.0$ & $31.0+-0.0$ & G1 & AD06 \\
\hline $0.1+-0.0$ & $1.0+-0.0$ & A2 & AD04 \\
\hline $0.1+-0.0$ & $14.0+-0.0$ & A2 & AD06 \\
\hline $0.1+-0.0$ & $1.0+-0.0$ & A1 & AD04 \\
\hline $0.1+-0.0$ & $8.0+-0.0$ & A1 & AD06 \\
\hline $0.04+-0.0$ & $1.0+-0.0$ & G2 & AD04 \\
\hline $0.04+-0.0$ & $47.0+-0.0$ & G2 & AD06 \\
\hline $0.14+-0.0$ & $1.0+-0.0$ & $\mathrm{~A} 1$ & AD04 \\
\hline $0.14+-0.0$ & $13.0+-0.0$ & A1 & AD06 \\
\hline $0.13+-0.0$ & $1.0+-0.0$ & A2 & AD04 \\
\hline $0.13+-0.0$ & $2.0+-0.0$ & A2 & AD06 \\
\hline $0.15+-0.0$ & $1.0+-0.0$ & G2 & AD04 \\
\hline $0.15+-0.0$ & $5.0+-0.0$ & G2 & AD06 \\
\hline $0.09+-0.0$ & $1.0+-0.0$ & G2 & AD04 \\
\hline $0.09+-0.0$ & $46.0+-0.0$ & G2 & AD06 \\
\hline $0.0+-0.0$ & $1.0+-0.0$ & G1 & AD04 \\
\hline $0.0+-0.0$ & $31.0+-0.0$ & G1 & AD06 \\
\hline $0.07+-0.0$ & $1.0+-0.0$ & G1 & AD04 \\
\hline $0.07+-0.0$ & $51.0+-0.0$ & G1 & AD06 \\
\hline $0.15+-0.0$ & $1.0+-0.0$ & G1 & AD04 \\
\hline $0.15+-0.0$ & $23.0+-0.0$ & G1 & AD06 \\
\hline $0.13+-0.0$ & $1.0+-0.0$ & A1 & AD04 \\
\hline $0.13+-0.0$ & $4.0+-0.0$ & A1 & AD06 \\
\hline $0.09+-0.0$ & $1.0+-0.0$ & $\mathrm{~A} 1$ & AD04 \\
\hline $0.09+-0.0$ & $10.0+-0.0$ & A1 & AD06 \\
\hline $0.03+-0.0$ & $1.0+-0.0$ & G2 & AD04 \\
\hline $0.03+-0.0$ & $36.0+-0.0$ & G2 & AD06 \\
\hline $0.1+-0.0$ & $1.0+-0.0$ & G1 & AD04 \\
\hline $0.1+-0.0$ & $52.0+-0.0$ & G1 & AD06 \\
\hline $0.09+-0.0$ & $1.0+-0.0$ & A2 & AD04 \\
\hline $0.09+-0.0$ & $17.0+-0.0$ & A2 & AD06 \\
\hline $0.08+-0.0$ & $1.0+-0.0$ & A2 & AD04 \\
\hline $0.08+-0.0$ & $3.0+-0.0$ & $\mathrm{~A} 2$ & AD06 \\
\hline $0.19+-0.0$ & $1.0+-0.0$ & $\mathrm{~A} 1$ & AD04 \\
\hline $0.19+-0.0$ & $5.0+-0.0$ & A1 & AD06 \\
\hline $0.08+-0.0$ & $1.0+-0.0$ & $\mathrm{~A} 2$ & $\mathrm{AD} 04$ \\
\hline $0.08+-0.0$ & $10.0+-0.0$ & $\mathrm{~A} 2$ & AD06 \\
\hline $0.11+-0.0$ & $1.0+-0.0$ & G2 & AD04 \\
\hline $0.11+-0.0$ & $15.0+-0.0$ & G2 & AD06 \\
\hline $0.05+-0.0$ & $1.0+-0.0$ & G2 & AD04 \\
\hline $0.05+-0.0$ & $31.0+-0.0$ & G2 & AD06 \\
\hline $0.08+-0.0$ & $1.0+-0.0$ & G2 & AD04 \\
\hline $0.08+-0.0$ & $31.0+-0.0$ & G2 & AD06 \\
\hline $0.06+-0.0$ & $1.0+-0.0$ & G2 & AD04 \\
\hline $0.06+-0.0$ & $36.0+-0.0$ & G2 & AD06 \\
\hline $0.12+-0.0$ & $1.0+-0.0$ & G1 & AD04 \\
\hline $0.12+-0.0$ & $2.0+-0.0$ & G1 & AD06 \\
\hline $0.03+-0.0$ & $1.0+-0.0$ & A2 & AD04 \\
\hline $0.03+-0.0$ & $15.0+-0.0$ & A2 & AD06 \\
\hline $0.05+-0.0$ & $1.0+-0.0$ & G1 & AD04 \\
\hline $0.05+-0.0$ & $6.0+-0.0$ & G1 & AD06 \\
\hline $0.07+-0.0$ & $1.0+-0.0$ & A2 & AD04 \\
\hline $0.07+-0.0$ & $4.0+-0.0$ & A2 & AD06 \\
\hline $0.11+-0.0$ & $1.0+-0.0$ & G1 & AD04 \\
\hline $0.11+-0.0$ & $17.0+-0.0$ & G1 & AD06 \\
\hline $0.05+-0.0$ & $1.0+-0.0$ & G1 & AD04 \\
\hline $0.05+-0.0$ & $34.0+-0.0$ & G1 & AD06 \\
\hline $0.15+-0.0$ & $1.0+-0.0$ & G2 & AD04 \\
\hline $0.15+-0.0$ & $4.0+-0.0$ & G2 & AD06 \\
\hline $0.11+-0.0$ & $1.0+-0.0$ & G2 & AD04 \\
\hline $0.11+-0.0$ & $46.0+-0.0$ & G2 & AD06 \\
\hline $0.11+-0.0$ & $1.0+-0.0$ & G1 & AD04 \\
\hline $0.11+-0.0$ & $29.0+-0.0$ & G1 & AD06 \\
\hline $0.09+-0.0$ & $1.0+-0.0$ & G1 & AD04 \\
\hline
\end{tabular}




\begin{tabular}{|c|c|c|c|c|c|c|c|}
\hline QVLGEADYSDSVGSYYVDYFDS & IGHV4-39 & IGHJ4 & 1 & $0.09+-0.0$ & $54.0+-0.0$ & G1 & AD06 \\
\hline EGDYGGNIYFQH & IGHV1-18 & IGHJ1 & 1 & $0.14+-0.0$ & $1.0+-0.0$ & $\mathrm{~A} 2$ & $\mathrm{AD} 04$ \\
\hline EGDYGGNIYFQH & IGHV1-18 & IGHJ1 & 1 & $0.14+-0.0$ & $15.0+-0.0$ & A2 & AD06 \\
\hline FDM & IGHV3-7 & IGHJ3 & 1 & $0.13+-0.0$ & $2.0+-0.0$ & $\mathrm{~A} 2$ & AD09 \\
\hline DRRFGELSVSTMDV & IGHV3-74 & IGHJ6 & 1 & $0.11+-0.0$ & $1.0+-0.0$ & G2 & AD09 \\
\hline DRRFGELSVSTMDV & IGHV3-74 & IGHJ6 & 11 & $0.11+-0.01$ & $1.55+-0.78$ & $\mathrm{G} 2 \mid \mathrm{A} 2$ & AD08 \\
\hline VSRMEARDY & IGHV3-7 & IGHJ4 & 1 & $0.07+-0.0$ & $1.0+-0.0$ & G2 & $\mathrm{AD} 04$ \\
\hline VSRMEARDY & IGHV3-7 & IGHJ4 & 2 & $0.08+-0.0$ & $4.5+-3.5$ & G2 & AD03 \\
\hline RAGGHSSGYHYYFDK & IGHV4-59 & IGHJ4 & 1 & $0.12+-0.0$ & $1.0+-0.0$ & A2 & $\mathrm{AD} 04$ \\
\hline RAGGHSSGYHYYFDK & IGHV4-59 & IGHJ4 & 2 & $0.11+-0.01$ & $36.0+-21.0$ & $\mathrm{~A} 2$ & $\mathrm{AD} 03$ \\
\hline DLATLEWLLSN & IGHV3-23 & IGHJ4 & 1 & $0.09+-0.0$ & $1.0+-0.0$ & G1 & AD04 \\
\hline DLATLEWLLSN & IGHV3-23 & IGHJ4 & 1 & $0.09+-0.0$ & $20.0+-0.0$ & G1 & AD03 \\
\hline SADYYHYYNGMDV & IGHV3-33 & IGHJ6 & 1 & $0.13+-0.0$ & $1.0+-0.0$ & A1 & $\mathrm{AD} 01$ \\
\hline HAGHLGLVLDI & IGHV3-7 & IGHJ3 & 2 & $0.09+-0.0$ & $19.0+-18.0$ & $\mathrm{G} 2$ & AD05 \\
\hline RYCSGGSCYSGIDY & IGHV3-11 & IGHJ4 & 1 & $0.01+-0.0$ & $1.0+-0.0$ & $\mathrm{~A} 2$ & AD09 \\
\hline RYCSGGSCYSGIDY & IGHV3-11 & IGHJ4 & 1 & $0.0+-0.0$ & $12.0+-0.0$ & A2 & AD04 \\
\hline DGGRVVGNTLPGY & IGHV3-7 & IGHJ4 & 1 & $0.03+-0.0$ & $1.0+-0.0$ & A1 & AD04 \\
\hline DGGRVVGNTLPGY & IGHV3-7 & IGHJ4 & 1 & $0.07+-0.0$ & $9.0+-0.0$ & $\mathrm{~A} 2$ & AD06 \\
\hline
\end{tabular}


A

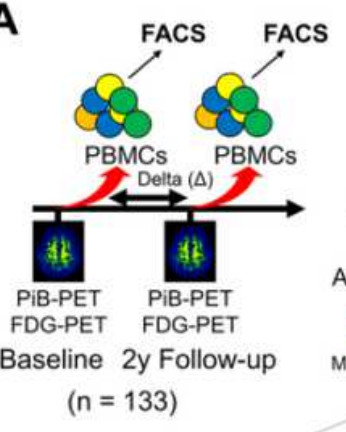

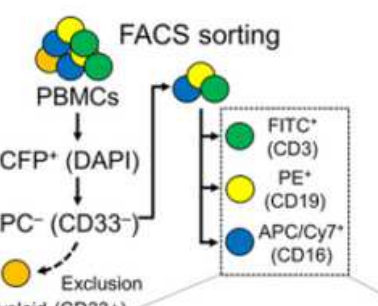

Human blood

Lymphocytic

Population

Profiling Myeloid (CD33+)
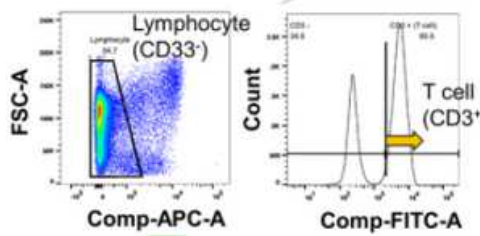

Multiple regression analysis

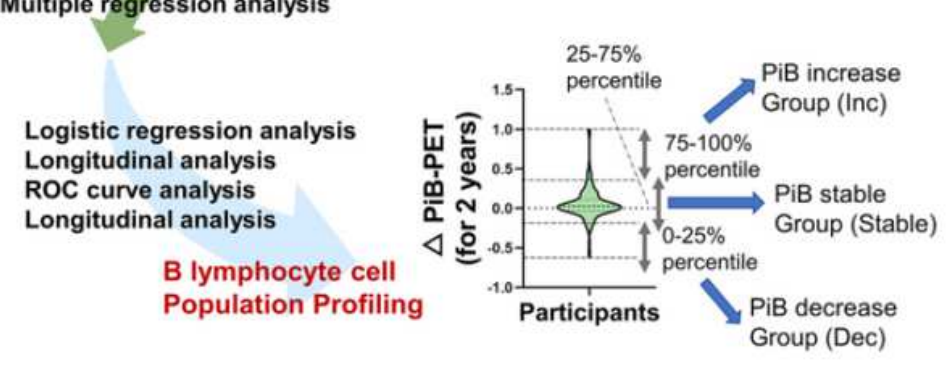

B

\begin{tabular}{|c|c|c|c|c|c|c|}
\hline \multicolumn{5}{|c|}{$\begin{array}{l}\text { Dependent variable: } \Delta \text { Global PiB } \\
\text { deposition (SUVR) }\end{array}$} & \multicolumn{2}{|c|}{$\begin{array}{l}\text { Method: } \\
\text { Stepwise }\end{array}$} \\
\hline $\begin{array}{l}\text { Indep. } \\
\text { variable }\end{array}$ & $\beta$ & SE & $\mathrm{t}$ & $\begin{array}{c}P \\
\text { value }\end{array}$ & $\begin{array}{l}\text { F-ratio } \\
\text { (DF) }\end{array}$ & $\begin{array}{l}\text { Adj- } \\
R^{2}\end{array}$ \\
\hline$\Delta \mathrm{B}$ cell & 0.0065 & 0.0017 & 3.698 & $0.0003^{\text {*** }}$ & $\begin{array}{c}13.67 \\
(1,131)\end{array}$ & 0.088 \\
\hline
\end{tabular}

C

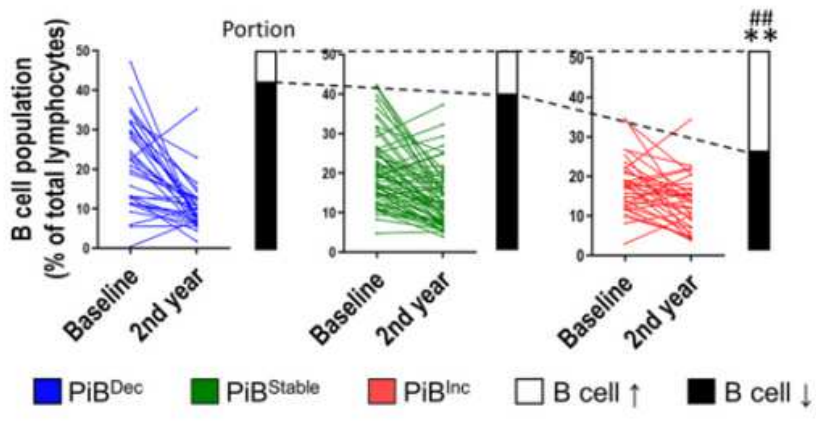

\section{Figure 1}

The strategy for longitudinal analysis (A) Timeline of the longitudinal study ( $n=133)$. Brain imaging and isolation of peripheral mononuclear cell (PBMC) were performed at both baseline (BL) and 2nd year follow-up (FL) time-point. Classification of the PBMCs by the FACS analysis (CD3+ for T lymphocytes, CD19+ for B lymphocytes, and CD16+ for NK lymphocytes). Participants were classified into three groups for the in-depth analyses (PiB increase group, PiBlnc, getting worse for 2 years; PiB stable group, PiBStable, none the worse for 2 years; PiB possible decrease group, PiBDec, relatively getting better for 2 years). (B) Step-wise multiple regression analysis between the delta $(\Delta)$ lymphocytes $(B, T, N K$ lymphocytes) and $\Delta$ global PiB deposition (standardized uptake value ratio; SUVR). Age and gender were also included as covariates. Only B lymphocyte is selected by the stepwise method. (C) B lymphocyte changes between baseline and follow-up 2nd year. PiBlnc group has higher portion of B cell increasedparticipants than PiBDec and PiBStable group (Chi-trend test, $\# \# p<0.01$; Chi-squared test, ${ }^{\star \star} p<0.01$ ). 
C
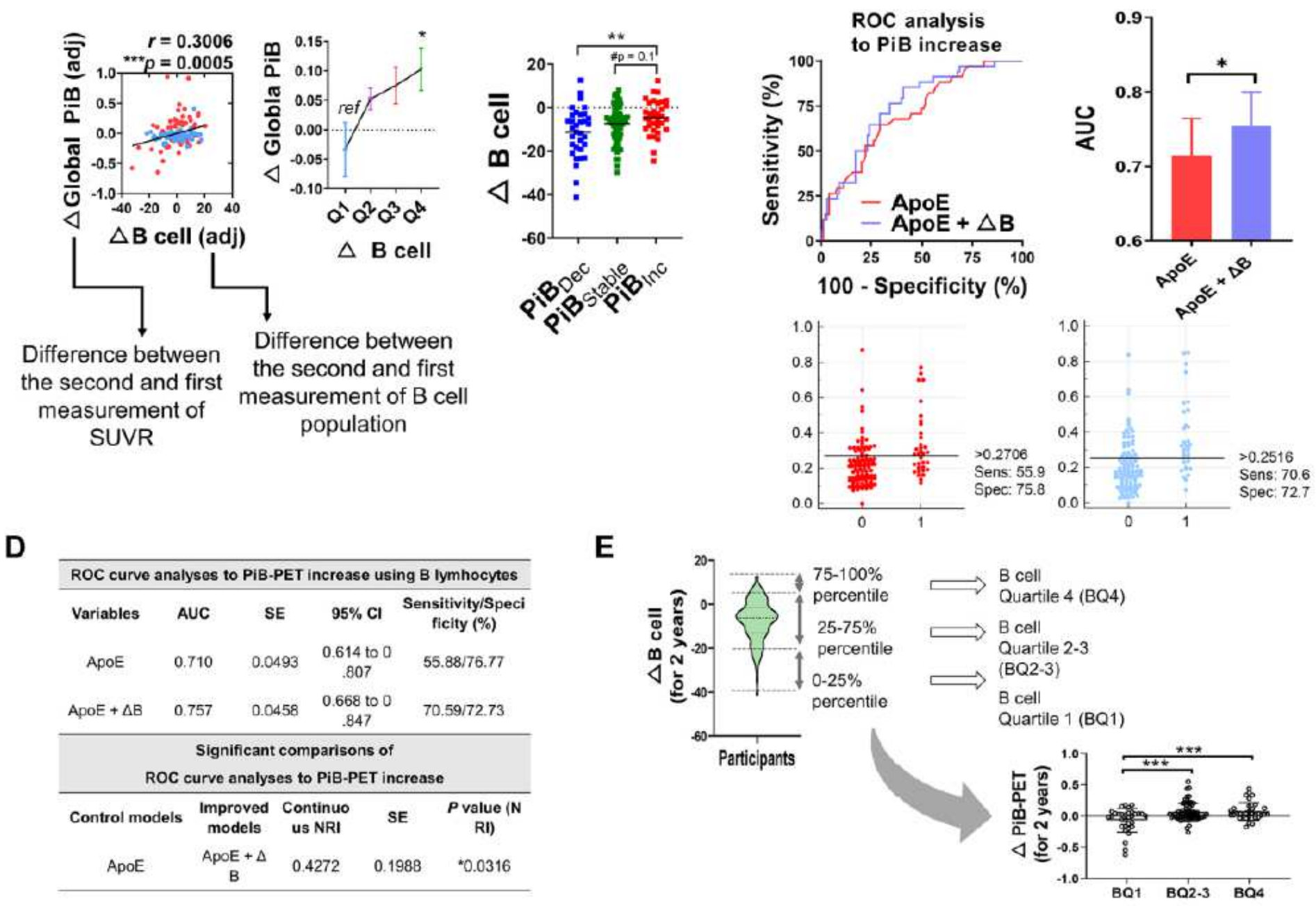

\section{Figure 2}

Longitudinal analysis and receiver operation curve (ROC) analysis for B lymphocyte profiling (A) Partial correlation analysis between $\Delta$ global PiB SUVR and $\Delta \mathrm{B}$ lymphocytes ( ${ }^{* \star} \mathrm{p}<0.001$, left) and ANOVA with post-hoc test between the quartiles of $\Delta \mathrm{B}$ lymphocytes $(\mathrm{Q} 1<\mathrm{Q} 2<\mathrm{Q} 3<\mathrm{Q} 4)$ and $\Delta$ global PiB SUVR. (B) PiBInc group significantly showed less decrease in B cell population than PiBDec group for 2 years ( $* \star p<$ 0.01, ANOVA with Tukey's post-hoc test). (C-D) Biomarkers of B cell showed better discrimination power to PiB-progression when combined with APOE genotyping. ${ }^{*} p<0.05$ by continuous net reclassification index (NRI). Interactive dot diagram showed sensitivity/specificity/cut-off (pi values by Youden index, $>0.2706$, $>0.2516$ ) values for PiB increase- ROC curves. (E) Participants were re-classified according to their quartiles of $\triangle B$ lymphocytes (BQ1<BQ2<BQ3<BQ4) and divided into three groups (BQ1, BQ2-3, BQ4). Delta PiB-PET values were significantly increased in BQ2-3 and BQ4 than BQ1 (*** $<<0.001$; ANOVA with Tukey's post-hoc test). Two outliers were excluded by Grubb's test. 
A

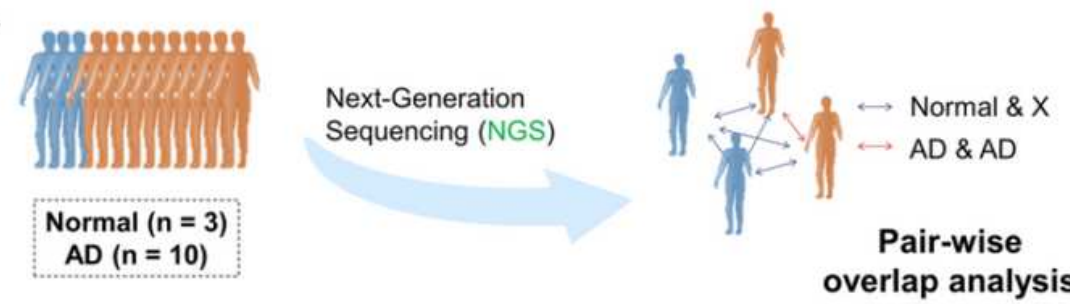

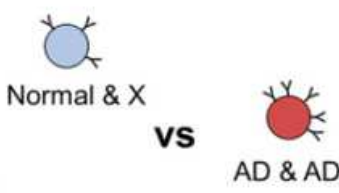

$B C R$ repertoire profiling
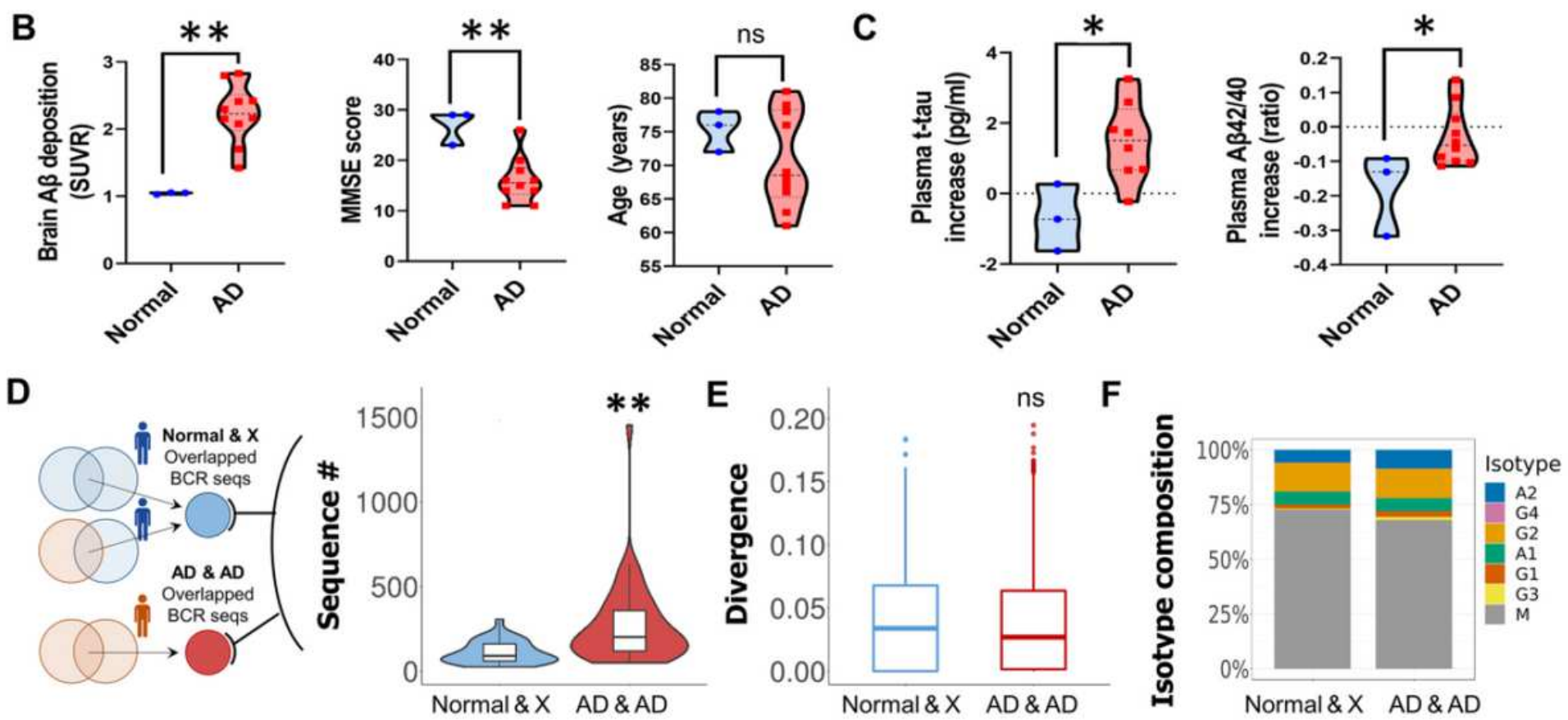

Figure 3

NGS-based BCR repertoire profiling followed by pair-wise BCR overlap analysis (A) B cell receptor (BCR) repertoire analysis $(n=13)$. Total of 13 participants (10 AD patients and 3 cognitively normals) were used for the BCR repertoire analysis. Overlapped BCR sequences were defined by pair-wise overlap analysis on $B C R$ repertoire of the samples, which was followed by comparative analysis between Normal\&X (comparison between Normal and Normal or Normal and AD; blue bidirectional arrow of the upper panel) and $A D \& A D$ (comparison between $A D$ and $A D$; red bidirectional arrow of the upper panel) groups. (B) Characterization of samples subjected to BCR repertoire analysis. ${ }^{*} p<0.05$ and ${ }^{* *} p<0.01$, independent $t-$ test. (C) The changes in the levels of plasma t-tau and beta-amyloid 42/40 ratio. ${ }^{*} p<0.05$, independent ttest. (D) The number of overlapped BCR sequences resulted from pair-wise overlap analysis. AD\&AD had more overlapped BCR sequences than Normal\&X. ${ }^{*} \mathrm{p}<0.001$; Wilcoxon rank-sum test. (E) Divergence of the overlapped BCR sequences. (F) Isotype composition of the overlapped BCR sequences. 
A
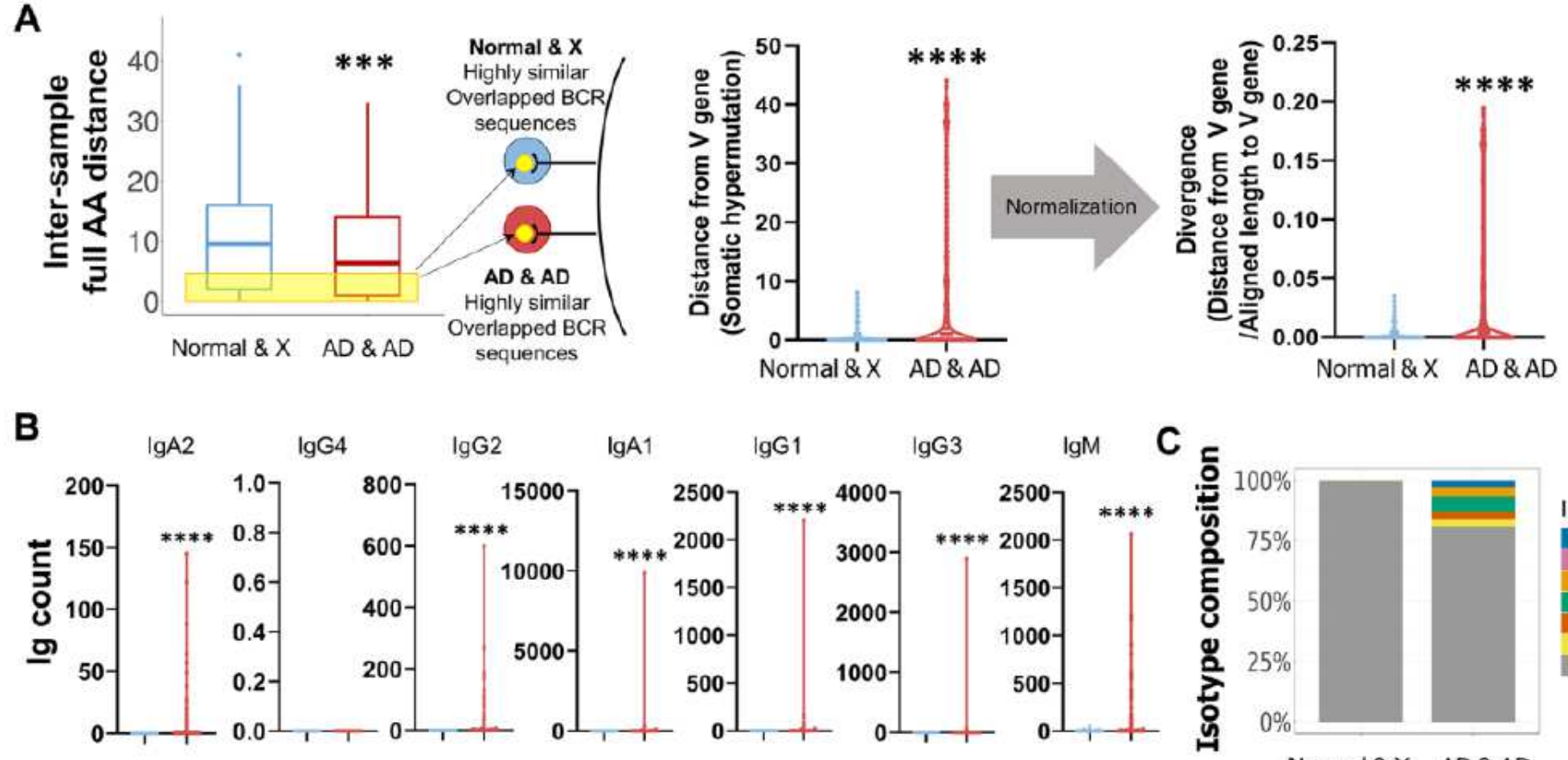

Normal \& $X \quad A D \& A D$

D

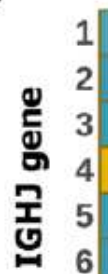
0.05
0.04
0.03
0.02
0.01
0.00

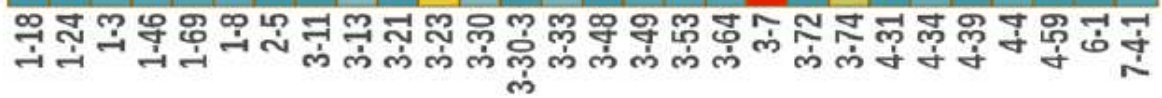
IGHV gene

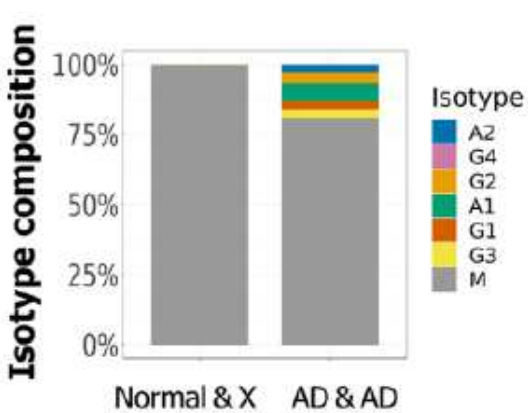

E

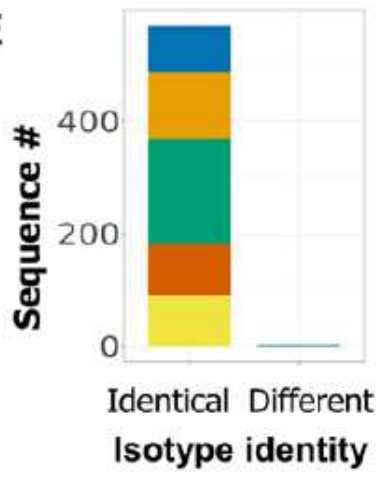

\section{Figure 4}

NGS-based BCR repertoire profiling followed by pair-wise BCR overlap analysis (high similar overlapped sequences) (A) Inter-sample full amino acid (AA) distance of the overlapped BCR sequences. The averaged hamming distance between BCR sequences, which were overlapped and derived from different samples, was calculated using full AA sequences. This distance was named as inter-sample full AA distance. Among the overlapped BCR sequences, sequences with inter-sample full AA distance value smaller than 5 were defined as 'highly similar overlapped BCR sequence'. Significantly increased divergence of highly similar overlapped BCR sequences in AD\&AD group. ${ }^{* \star \star} \mathrm{p}<0.0001$; Wilcoxon ranksum test. (B) The number of Ig counts between Normal\&X vs AD\&AD. ****p < 0.0001; Man-Whitney test. (C) Isotype composition of the highly similar overlapped BCR sequences. Despite of a small inter-sample full AA distance, the sequences with a high divergence were only shown in AD\&AD. (D) VJ gene usage of the class-switched and highly similar overlapped BCR sequences. (E) Isotype identity of the classswitched and highly similar overlapped BCR sequences. Matches in isotypes in different samples of the class-switched and highly similar overlapped BCR sequences were quantified. If the isotypes of 
sequences were identical in different two samples, they were counted as "identical". And, if not, they were counted as "different". The isotype of the sequences was also represented by colors following the color coding used in $(\mathrm{C})$.

A

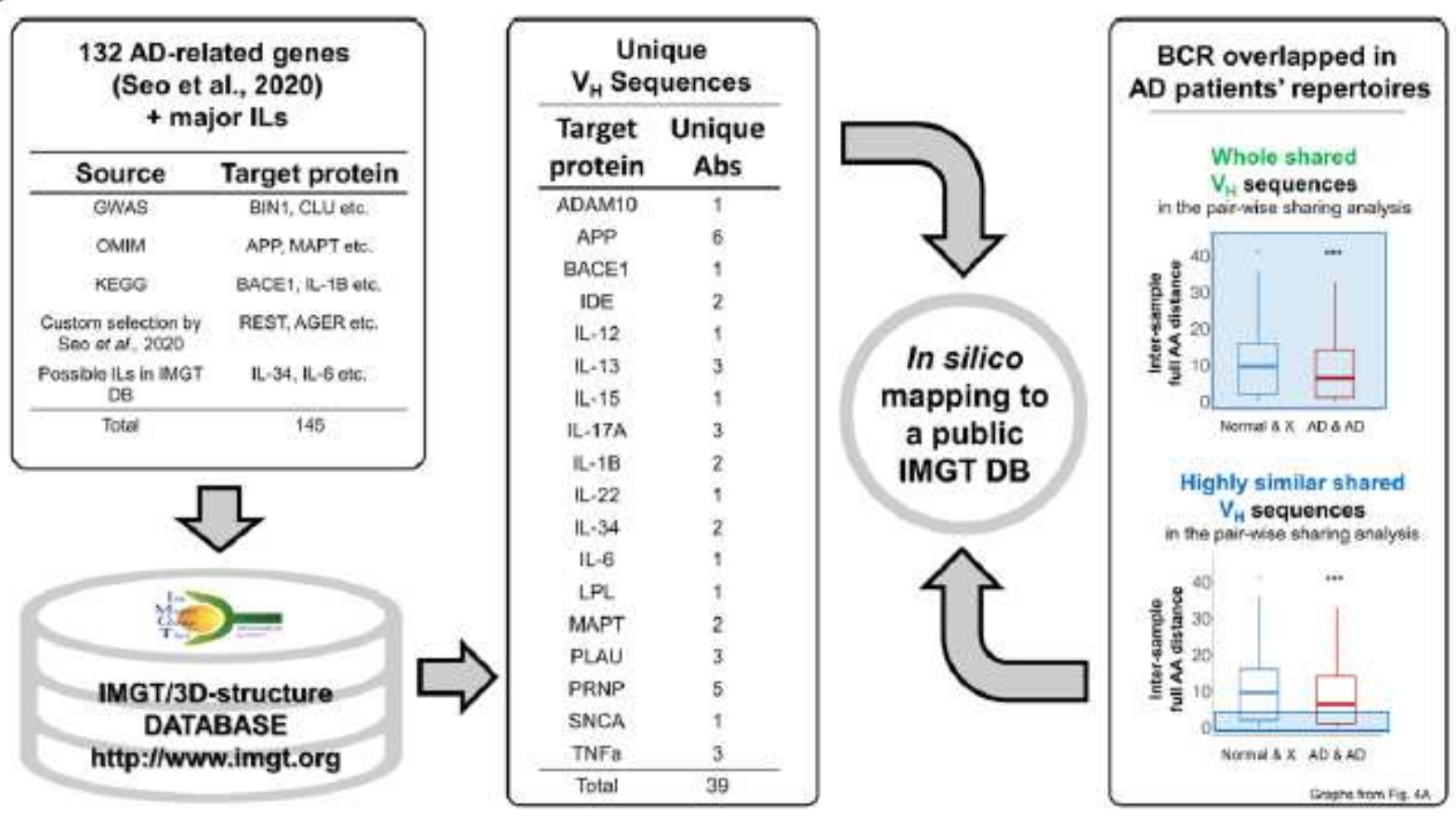

B

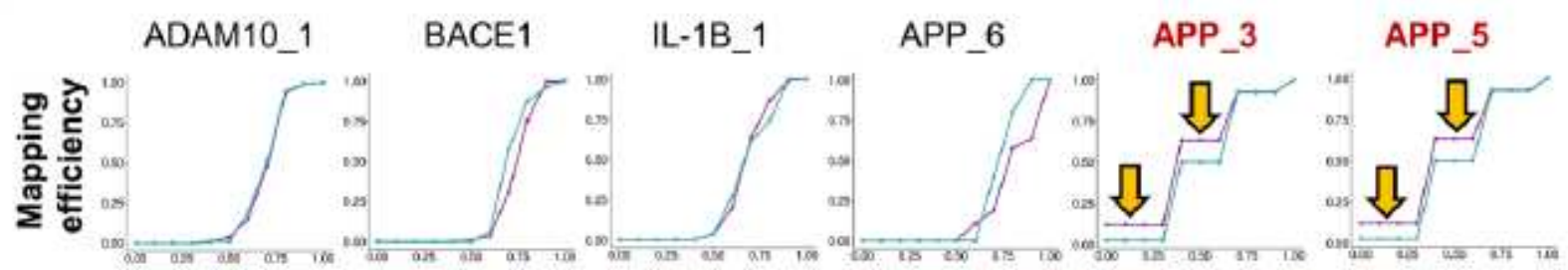

CDR3 AA mismatch threshold for whole shared $V_{H}$ sequences

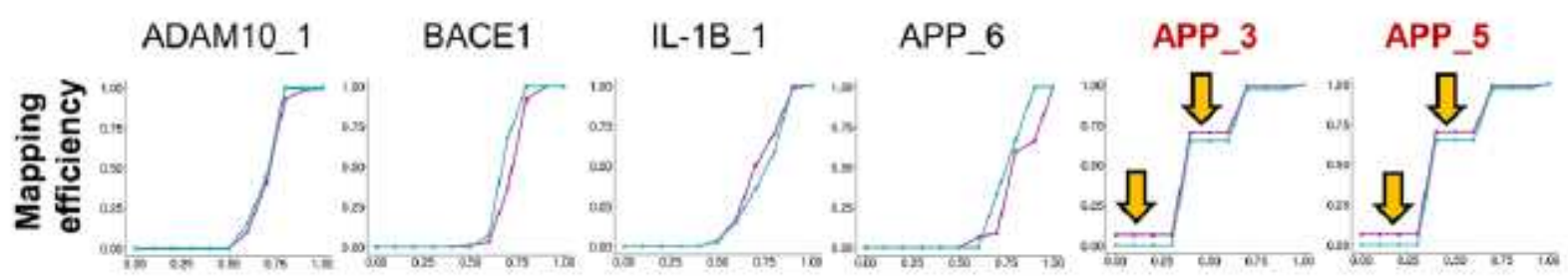

CDR3 AA mismatch threshold for highly similar shared $V_{H}$ sequences

C

- $A D \& A D$ - Normal \& $X$

\begin{tabular}{|l|l|l|l|l|l|r|}
\hline \multicolumn{1}{|c|}{ seq_id } & target_protein & CDR1_AA & CDR2_AA & CDR3_AA & V_gene & divergence \\
\hline APP_3 & APP & GFTFSRYS & INSVGSST & GDY & IGHV3-23*05 & 11.34 \\
\hline APP_5 & APP & GFTFSRYS & INSVGNST & GDY & IGHV3-23*04 & 12.37 \\
\hline
\end{tabular}

APP-specific VH sequence from IMGT database

Figure 5

In silico mapping of the overlapped BCR sequences to antibodies binding to AD-related genes (A) Experimental flow chart for In silico mapping analysis. The Immunogenetics information system (IMGT) 
database was used for the generation of antibody sequences (unique VH sequences). (B) The mapping results from the comparison between the whole or highly shared $\mathrm{VH}$ sequences and the unique $\mathrm{VH}$ sequences. The APP-binding antibodies showed different mapping patterns between AD\&AD and Normal\&X. (C) The information of APP-specific VH sequences from the IMGT database.

Control
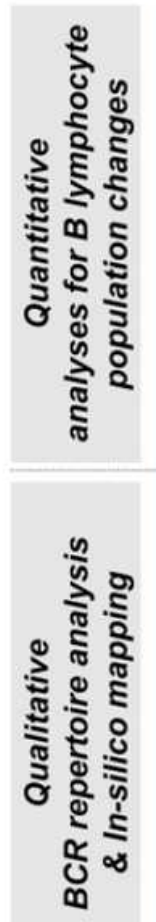

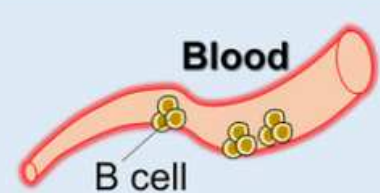

The decrease in B cell population by Aging (Normal status)
Longitudinal analysis

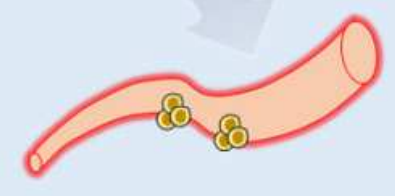

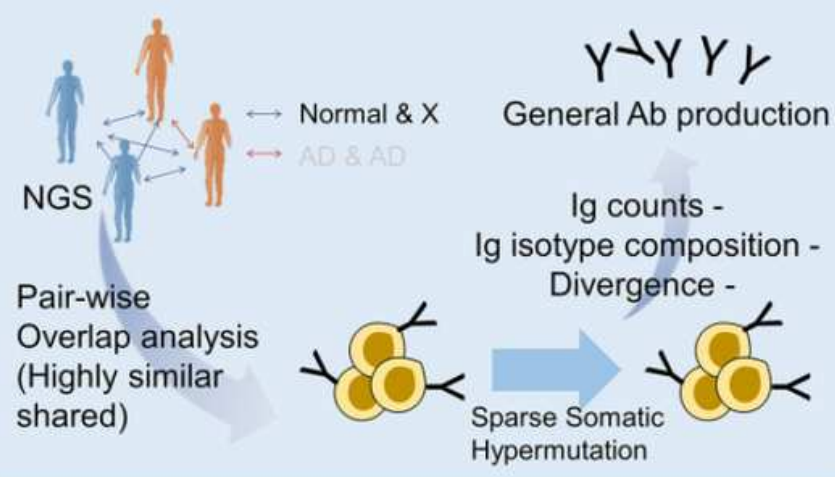

Alzheimer's Disease

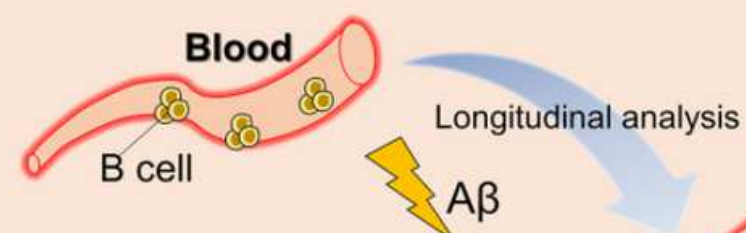

The increase in B cell population in $A D$ (Abnormal status)

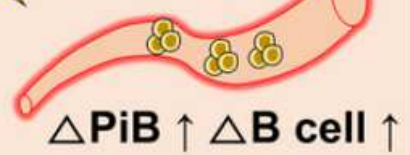

$76 \%$ Accuracy with $71 \%$ Sen and $73 \%$ Spe

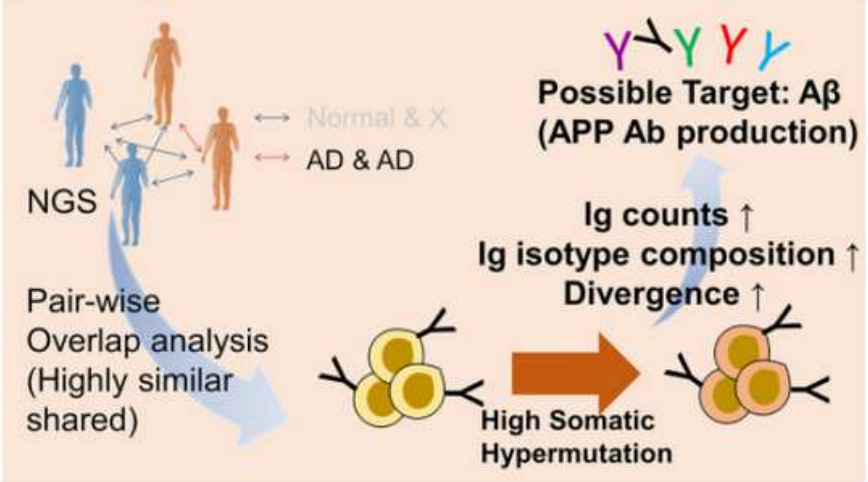

\section{Figure 6}

A graphical summary of this study From the quantitative and longitudinal analysis, we found that the population change in $B$ lymphocyte over two years is associated with the increase in cerebral betaamyloid deposition in AD. Furthermore, from the qualitative BCR repertoire analysis and in-silico mapping results, we identified that the commonalities of BCR repertoires within AD patients (Ig counts, Ig isotype composition, divergence) and suggested the possibility of the immunological reaction to amyloid precursor protein (APP). NGS, next-generation sequencing; Ab, antibody; $A \beta$, beta-amyloid; APP, amyloid precursor protein; Ig, immunoglobulin. 